\title{
Nuclear NFATc1/Smad3 complexes in Smad4-deficient pancreatic cancer
}

\author{
DISSERTATION \\ for the award of the degree \\ Doctor rerum naturalium (Dr. rer. nat.) \\ of the Georg-August Universität Göttingen
}

within the doctoral program Molecular Medicine

of the Georg-August University School of Science (GAUSS)

submitted by

Marie Christin Hasselluhn

born in Lauchhammer, Germany

Göttingen, 2019 



\section{Thesis Advisory Committee}

PD Dr. med. Elisabeth Heßmann, Department of Gastroenterology and Gastrointestinal Oncology, University Medical Center Göttingen

Prof. Dr. med. Heidi Hahn, Department of Human Genetics, University Medical Center Göttingen

Prof. Dr. med. Matthias Dobbelstein, Institute of Molecular Oncology, University Medical Center Göttingen

\section{Members of the Examination Board}

$1^{\text {st }}$ Referee: PD Dr. med. Elisabeth Heßmann, Department of Gastroenterology and Gastrointestinal Oncology, University Medical Center Göttingen

$2^{\text {nd }}$ Referee: $\quad$ Prof. Dr. med. Heidi Hahn, Department of Human Genetics, University Medical Center Göttingen

\section{Further members of the Examination Board}

Prof. Dr. med. Matthias Dobbelstein, Institute of Molecular Oncology, University Medical Center Göttingen

Prof. Dr. med. Michael P. Schön, Department of Dermatology, Venereology and Allergology, University Medical Center Göttingen

Prof. Dr. rer. nat. Dieter Kube, Department of Hematology and Oncology, University Medical Center Göttingen

Dr. rer. nat. Ufuk Günesdogan, Department of Developmental Biology, Georg-August Universität Göttingen

Date of oral examination: 21 May 2019 
What kept me sane was knowing

that things would change,

and it was a question of keeping myself together until they did.

NinA Simone 


\section{Affidavit}

I hereby declare that the PhD thesis entitled "Nuclear NFATc1/Smad3 complexes in

Smad4-deficient pancreatic cancer" has been written independently and with no other sources and aids than quoted.

Marie Christin Hasselluhn

March 2019

Göttingen 



\section{Abstract}

Until today, PDAC is characterized by its extremely high mortality rate and the lack of effective treatment strategies despite reinforced focus on the elucidation of underlying molecular mechanisms in the last decades. The development of novel chemotherapeutic treatment strategies remains a challenge due to its extraordinary heterogeneity with regards to genetic alterations, stromal composition, and tumor cell metabolism. Patient stratification according to molecular subtyping is a promising strategy to efficiently target PDAC in a precision medicine-based approach. Molecular taxonomy aims to exploit specific cancer vulnerabilities to increase chemotherapeutic response and prolong the life expectancy of patients.

In this work, we focused on the large genetic subgroup of SMAD4-deficient PDAC in the context of oncogenic KRAS activation, comprising $55 \%$ of patients. With the help of GEMMs, we aimed to comprehend features of Smad4-depleted PDAC in vivo and elucidate synergisms in Kras and TGF $\beta$ signaling. This is complemented by in vitro studies, opposing Smad4 WT PDAC cells to their respective CRISPR/Cas9-generated Smad4-depleted counterparts. We disclosed induced migratory potential and stemness characteristics as well as increased tolerance to the nucleoside analog Gemcitabine upon loss of Smad4. In contrast, Smad4-depleted PDAC cells demonstrated extraordinary sensitivity towards Mek inhibition by Trametinib.

The TGF $\beta$ pathway is strongly intertwined with NFATc1 signaling, an inflammatory transcription factor pivotal for PDAC evolution. We scrutinized the role of NFATc1 in hijacking Smad3-dependent transcription in favor of oncogenic gene signatures in Smad4 deficiency. Cooperativity studies illuminated direct interplay of NFATc1 and Smad3 transcription factors and conditions permitting or abrogating their nuclear complex formation. With the identification of cJun as a third transcription factor involved in the nuclear complex, we gained a window of opportunity to target the transcription complex by disrupting its assembly with Trametinib. Genome-wide expression studies 
revealed potential targets of the NFATc1/Smad3/cJun complex and disclosed transcriptional induction of genes associated with improved PDAC patient survival and increased Gemcitabine response upon complex disruption by Trametinib.

Thus, on the one hand, we suggest a mechanism how Smad4-depleted cells evade Gemcitabine-induced cytotoxicity by NFATc1/Smad3/cJun-mediated transcription. On the other hand, we propose a strategy on how to interfere with the transcription factor assembly by effective Mek inhibition mediated by Trametinib, thus restoring Gemcitabine sensitivity in the context of Smad4 deficiency. Together, these data provide a potential novel chemotherapeutic treatment regime with combinatorial Trametinib and Gemcitabine administration for Kras-mutated PDAC patients bearing loss of SMAD4. 


\section{Contents}

Affidavit

Abstract vii

List of Figures $\quad$ xiii

List of Tables $\quad$ xv

List of Abbreviations $\quad$ xvii

1 Introduction $\quad 1$

1.1 PDAC heterogeneity: chance and pitfall . . . . . . . . . . . . . 1

1.2 NFATc1 as an inflammatory driver of pancreatic carcinogenesis . . . . 4

1.3 TGF $\beta$ signaling in PDAC: a double-edged sword . . . . . . . . . 6

1.3.1 Canonical and non-canonical TGF $\beta$ pathway . . . . . . . . 7

1.3.2 Cooperativity of NFAT and TGF $\beta$ signaling cascades . . . . . . 11

1.4 Mechanisms behind Kras-driven PDAC . . . . . . . . . . . . . . . . . . 12

1.5 Aims of the study . . . . . . . . . . . . . . . . . . . 14

2 Material and Methods $\quad 17$

2.1 Cell culture . . . . . . . . . . . . . . . . . . . . . . . . . . . . . . 18

2.1.1 PDAC cells and cultivation . . . . . . . . . . . . 18

2.1.2 Treatment and transfection . . . . . . . . . . . . . . 19

2.1.3 CRISPR/Cas9-mediated Smad4 knock out . . . . . . . . . . . . 20

2.2 Functional assays . . . . . . . . . . . . . . . . . . . . . . . . 22

2.2.1 Annexin $\mathrm{V}$ and propidium iodide staining . . . . . . . . 22

2.2 .2 Migration assay . . . . . . . . . . . . . . . . . 23

2.2.3 MTT assay . . . . . . . . . . . . . . . . . . 23

2.2.4 Cell counting assay . . . . . . . . . . . . . . . . . 24

2.2 .5 Soft agar assay . . . . . . . . . . . . . . . . . 24 
2.2.6 Sphere assay . . . . . . . . . . . . . . . . . . . . . . . . . 24

2.3 Molecular methods . . . . . . . . . . . . . . . . . 25

2.3.1 Protein isolation .................. 25

2.3.2 Co-immunoprecipitation . . . . . . . . . . . . . . 26

2.3.3 Western Blot . . . . . . . . . . . . . . . . . . 27

2.3.4 RNA isolation . . . . . . . . . . . . . . . . 30

2.3 .5 qRT-PCR . . . . . . . . . . . . . . 31

2.4 RNA sequencing and bioinformatic analyses . . . . . . . . . . . . 33

2.5 Genetically engineered mouse models . . . . . . . . . . . . . . . . 34

2.5.1 GEMM breeding .................. 34

2.5.2 Tissue processing . . . . . . . . . . . . . . . . . 37

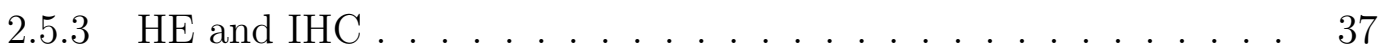

2.5 .4 IF and PLA . . . . . . . . . . . . . . . . . . 39

3 Results $\quad 41$

3.1 Nuclear NFATc1/Smad3 complexes in Smad4-deficient PDAC . . . . . 41

3.1.1 A murine model of Smad4 deficiency recapitulates features of human PDAC . . . . . . . . . . . . . . . 42

3.1.2 NFATc1 and Smad3 cooperate in Smad4 deficiency . . . . . . . 49

3.1.3 Smad4 deficiency favors nuclear NFATc1/Smad3 complex assembly 53

3.2 Targeting NFATc1/Smad3/cJun complexes as a novel therapeutic approach in $\mathrm{PDAC} \ldots \ldots \ldots \ldots$. . . . . . . . . . . . . . . . 62

3.2.1 AP-1 proteins contribute to NFATc1/Smad3 complex formation 62

3.2.2 Elucidation of NFATc1/Smad3/cJun regulated gene signatures in $\mathrm{PDAC} \ldots \ldots \ldots \ldots \ldots$ 
4 Discussion

4.1 Smad4 deficiency in pancreatic cancer favors invasiveness and aggressiveness of disease . . . . . . . . . . . . . . . . . . . . 83

4.1.1 Smad4 depletion cooperates with oncogenic Kras activation in strong acceleration of PDAC formation . . . . . . . . . . 84

4.1.2 Advantages of CRISPR/Cas9-mediated Smad4 knock out in PDAC research .................... 86

4.2 Smad4 status determines chemotherapeutic response in PDAC . . . . . 92

4.3 Concluding remarks . . . . . . . . . . . . . . . . . . 100

References 103

Appendix XXV 



\section{List of Figures}

1 PDAC arises from preneoplastic lesions . . . . . . . . . . . . . 2

2 Canonical and non-canonical TGF $\beta$ signaling $\ldots \ldots \ldots . . \ldots 7$

$3 \quad$ NFATc1 as an alternative Smad3 binding partner in Smad4-deficient

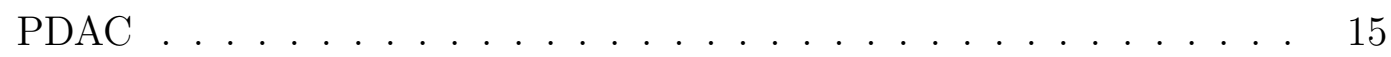

$4 \quad$ PDAC-associated survival is decreased upon Smad4 deficiency . . . . . 43

5 Homozygous Smad4 depletion abrogates epithelial Smad4 protein expression in the pancreas . . . . . . . . . . . . . . . 44

6 Smad4 deficiency accelerates formation of preneoplastic lesions and PDAC 45

7 Smad4 deficiency accelerates gain of relative pancreas weight . . . . . . 46

8 Smad4 deficiency hastens PDAC formation . . . . . . . . . . . . . . 46

9 Homozygous loss of Smad4 favors metastasis formation in low grade

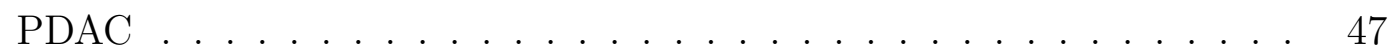

10 Nfatc1/Smad3 double positive tumor cells in Smad4 deficiency . . . . . 49

11 Nfatc1/Smad3 complexes in PDAC and immune cells . . . . . . . . . 50

12 NFATc1 cooperates with SMAD3 in SMAD4-deficient human PDAC. . 51

13 Establishment of CRISPR/Cas9-mediated Smad4 knock out in NKC-II

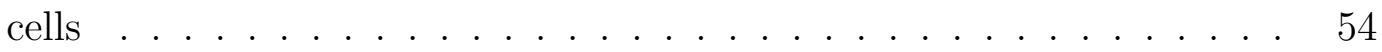

14 Smad4-dependent functional consequences of TGF $\beta$ treatment . . . . . 56

15 Smad4 dependency of nuclear NFATc1/Smad3 complex formation . . . 57

16 TGF $\beta$ dependency of nuclear NFATc1/Smad3 complex formation . . . 59

17 NFATc1/SMAD3 complex assembly depends on SMAD4 deficiency and oncogenic KRAS activation . . . . . . . . . . . . . . 60

18 NFATc1 forms transcription complexes with Smad3 and AP-1 proteins 63

19 cJun contributes to NFATc1/Smad3 complex formation . . . . . . . . . 64

20 Mek inhibition by UO126 disrupts nuclear NFATc1/Smad3/cJun com-

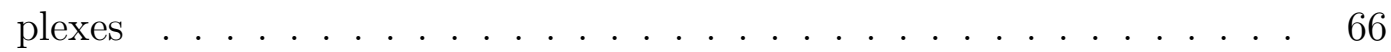


21 Mek inhibition by Trametinib disrupts nuclear NFATc1/Smad3/cJun complexes .............................. 67

22 Smad4 deficiency promotes Trametinib sensitivity in murine PDAC cells 68

23 Smad4 status determines Trametinib response in PDAC cells . . . . . . 70

24 PCA plot of distinct RNAseq conditions . . . . . . . . . . . . 71

25 Smad4 deficiency alters tumor cell morphology and their interactive potential ................................ 72

26 NFATc1/Smad3/cJun complexes interfere with proliferation, cell motility and ECM composition . . . . . . . . . . . . . . . 74

27 NFATc1/Smad3/cJun complex disruption interferes with dismal prognostic factors . . . . . . . . . . . . . . 76

28 Smad4 deficiency enhances Gemcitabine tolerance in murine PDAC cells 77

29 Trametinib administration restores Gemcitabine susceptibility . . . . . 79

30 Smad4 deficiency interferes with Gemcitabine toxicity . . . . . . . . . . 93

31 Trametinib targets oncogenic NFATc1/Smad3/cJun complexes in Smad4deficient PDAC . . . . . . . . . . . . . . . . 101

32 Proliferative capacity is not influenced by Smad4 status of PDAC cells xxv

33 Smad4 deficiency enhances Gemcitabine and AraC tolerance in PDAC

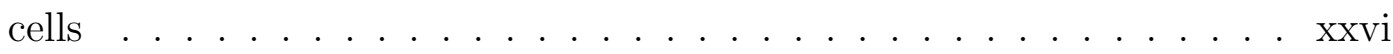

34 Induced Gemcitabine tolerance in KPC Bl6 cells is not mediated by Smad4 silencing . . . . . . . . . . . . . . . . . . xxvii 


\section{List of Tables}

1 Sequences of siRNAs . . . . . . . . . . . . . . . . . 19

2 sgRNAs designed for CRISPR/Cas9-mediated knock out . . . . . . . 21

3 Primer sequences for confirmation of Smad4 knock out . . . . . . . . . 22

4 Antibodies for co-immunoprecipitation experiments . . . . . . . . . 27

5 Composition of gel solutions . . . . . . . . . . . . . . . . . . . 28

6 Buffers for co-IP and SDS-PAGE . . . . . . . . . . . . . . . 29

7 Antibodies for western blot . . . . . . . . . . . . . . 30

8 Reaction mix for qRT-PCR experiments . . . . . . . . . . . . . . . . 32

9 Primer sequences for qRT-PCR experiments . . . . . . . . . . . . . 32

10 Primer sequences for genotyping . . . . . . . . . . . . . 35

11 Reaction mix for genotyping PCR . . . . . . . . . . . . . . . 36

12 PCR protocols . . . . . . . . . . . . . . . . . 36

13 Composition of antigen retrieval buffers . . . . . . . . . . . . 38

14 Composition of washing buffer . . . . . . . . . . . . . . . 38 



\section{List of Abbreviations}

adj.

ADM

AKT

AP-1

Aqua dest.

AraC

BMP

bp

BSA

Cas

Cda

Cdc20

cDNA

CDT

ChIP

ChIPseq

Cks1b

co-IP

Col7a1 adjusted

acinar-to-ductal metaplasia

RAC-alpha serine/threonine-protein kinase

activator protein 1

aqua destillata

cytosine arabinoside

bone morphogenetic protein

base pair

bovine serum albumin

CRISPR-associated

cytidine deaminase

cell division cycle protein 20 homolog

complementary deoxyribonucleic acid

si ctrl DMSO TGF $\beta$

chromatin immunoprecipitation

chromatin immunoprecipitation sequencing

cyclin-dependent kinases regulatory subunit 1

co-immunoprecipitation

collagen alpha-1 (VII) chain 
CRISPR clustered regularly interspaced short palindromic repeats

CST Cell Signaling Technology

ctrl control

CTT si ctrl Trametinib TGF $\beta$

DAB 3,3'-diaminobenzidine

DAPI 4',6-diamidino-2-phenylindole

Dck deoxycytidine kinase

Dctd deoxycytidylate deaminase

dFdC Gemcitabine or 2',2'-difluoro 2'-deoxycytidine

dFdCMP Gemcitabine monophosphate

dFdCTP Gemcitabine triphosphate

dFdU 2'2'-difluoro 2'-deoxyuridine

dFdUMP 2'2'-difluoro 2'-deoxyuridine monophosphate

DMEM Dulbecco's modified Eagle's medium

DMSO dimethyl sulfoxide

DNA deoxyribonucleic acid

DPC4 deleted in pancreatic carcinoma, locus 4

E2f1 E2f transcription factor 1

ECL enhanced chemiluminescence

ECM extracellular matrix 
E.coli

EDTA

EGF

EGFR

EGTA

EMT

ERK

FACS

FBS

FDA

FDR

FGF

Fgfbp1

fl/ +

$\mathrm{fl} / \mathrm{fl}$

FPKM

GDP

GEMM

GFP

GO
Escherichia coli

ethylenediaminetetraacetic acid

epidermal growth factor

epidermal growth factor receptor

ethylene glycol-bis( $\beta$-aminoethyl ether)-N,N,N',N'-tetraacetic acid

epithelial-mesenchymal transition

extracellular signal-regulated kinase

fluorescence-activated cell sorting

fetal bovine serum

food and drug administration

false discovery rate

fibroblast growth factor

fibroblast growth factor-binding protein 1

flox $/+$ (heterozygous)

flox/flox (homozygous)

fragments per kilobase million

guanosine diphosphate

genetically engineered mouse model

green fluorescent protein

gene ontology 


\begin{tabular}{|c|c|}
\hline GSEA & gene set enrichment analysis \\
\hline GTP & guanosine triphosphate \\
\hline $\mathrm{HE}$ & hematoxylin and eosin \\
\hline HEPES & 4-(2-hydroxyethyl)-1-piperazineethanesulfonic acid \\
\hline HRP & horseradish peroxidase \\
\hline $\mathbf{I C}_{50}$ & half maximal inhibitory concentration \\
\hline IF & immunofluorescence \\
\hline IgG & immunoglobulin $\mathrm{G}$ \\
\hline IHC & immunohistochemistry \\
\hline JNK & cJun N-terminal kinase \\
\hline KC p48 & $\operatorname{Kras}^{G 12 D} ;$ p48-Cre \\
\hline k.o. & knock out \\
\hline KPC & $\operatorname{Kras}^{G 12 D} ; p 53^{R 172 C /+} ;$ pdx-Cre \\
\hline KPC Bl6 & $\operatorname{Kras}^{G 12 D} ; p 53^{R 172 C /+} ; p d x-C r e$ \\
\hline KRAS & Kirsten rat sarcoma \\
\hline KLF10 & Krüppel-like factor 10 \\
\hline LB & lysogeny broth \\
\hline Ldlr & low-density lipoprotein receptor \\
\hline $\mathrm{LOH}$ & loss of heterozygosity \\
\hline
\end{tabular}


Mcm4

MEK

MH

$\operatorname{miR}$

Mmp

mRNA

MTT

NDT

NEAA

NES

NFAT

NFATc1

NKC p48

NLS

Nt5c

$\mathrm{Nt5c2}$

Nt5c3

$\mathbf{o} / \mathbf{n}$

PanIN

PANTHER minichromosome maintenance complex component 4

mitogen-activated protein kinase kinase

mad homology

micro RNA

matrix metalloproteinase

messenger ribonucleic acid

methylthiazolyldiphenyl tetrazolium bromide

siNFATc1 DMSO TGF $\beta$

non-essential amino acids

normalized enrichment score

nuclear factor of activated $\mathrm{T}$ cells

nuclear factor of activated $\mathrm{T}$ cells, cytoplasmic 1

cnNFATc1; Kras ${ }^{\mathrm{G} 12 \mathrm{D}}$; p48-Cre

nuclear localization sequence

5'(3')-deoxyribonucleotidase, cytosolic type

cytosolic purine 5'-nucleotidase

cytosolic 5'-nucleotidase $3 \mathrm{~A}$

overnight

pancreatic intraepithelial neoplasia

protein analysis through evolutionary relationships 
PB

PBS

PCA

PCR

PDAC

PDK1

PDX

PI3K

PLA

qRT-PCR

RNA

RNAseq

RPMI

Rrm1

Rrm2

RT

S4ko

S4WT

SBE

SDS phosphate buffer

phosphate buffered saline

principal component analysis

polymerase chain reaction

pancreatic ductal adenocarcinoma

3-phosphoinositide-dependent protein kinase 1

patient-derived xenograft

phosphatidylinositol 3 kinase

proximity ligation assay

quantitative real time polymerase chain reaction

ribonucleic acid

RNA sequencing

Roswell Park Memorial Institute

ribonucleoside-diphosphate reductase large subunit

ribonucleoside-diphosphate reductase subunit M2

room temperature

NKC-II Smad4 knock out

NKC-II Smad4 wildtype

Smad binding element

sodium dodecyl sulfate 
SDS-PAGE sodium dodecyl sulfate polyacrylamide gel electrophoresis

sgRNA single guide RNA

siRNA small interfering RNA

SKC $\mathbf{H} /+\mathbf{p} 48 \quad \operatorname{Smad}^{\mathrm{H} /+} ; \mathrm{KraS}^{G 12 D} ;$ p48-Cre

SKC $\boldsymbol{f l} / \boldsymbol{f l} \mathbf{p 4 8} \quad \mathrm{Smad} 4^{\mathrm{H} / \mathrm{fl}} ; \mathrm{Kras}^{\mathrm{G12D}} ;$ p48-Cre

Slc29a1 equilibrative nucleoside transporter 1

TBS Tris-buffered saline

TCGA The Cancer Genome Atlas

TE Tris EDTA

TGF $\beta \quad$ transforming growth factor beta

TMA tissue microarray

Tris tris(hydroxymethyl)-aminomethan

Tuba1c tubulin alpha-1C chain

$\mathbf{T} \beta \mathbf{R} \quad$ TGF $\beta$ receptor

UO126 1,4-diamino-2,3-dicyano-1, 4-bis[2-aminophenylthio]butadiene

USA United States of America

$\mathbf{v} / \mathbf{v} \quad$ volume per volume

$\mathbf{w} / \mathbf{v} \quad$ weight per volume

WCL whole cell lysate

WT wildtype 



\section{Introduction}

Pancreatic cancer is a highly lethal disease that is projected to become the second leading cause of cancer-related death in the United States of America (USA) and Germany by 2030 (Rahib et al., 2014; Quante et al., 2016). Its dismal prognosis is substantiated by late diagnosis due to unspecific symptoms, pronounced plasticity, extreme heterogeneity, and strong resistance to chemotherapeutic treatment (Adamska et al., 2017; Siegel et al., 2018). The 5 year survival of $8 \%$ covers all stages of the disease and represents the lowest score in cancer statistics of the USA (Siegel et al., 2018). Despite reinforced elucidation of molecular mechanisms driving pancreatic ductal adenocarcinoma (PDAC) formation and progression, there is still no breakthrough in advanced cancer therapies. Due to exceptional genetic heterogeneity, PDAC research is now focusing on patient stratification to offer customized therapeutic approaches for molecular subtypes of this dreadful disease.

\subsection{PDAC heterogeneity: chance and pitfall}

The most prevalent variant of pancreatic cancer accounting for $90 \%$ of patient cases originates from the exocrine gland and is termed PDAC (Biankin et al., 2012). Hahn \& Kern (1995) first identified Kirsten rat sarcoma (KRAS) as a crucial enhancer of PDAC formation and progression. In over $90 \%$ of invasive PDAC, activating mutations of the KRAS protooncogene are described, predominantly mediated by G12D transition (Hingorani et al., 2003; Bryant et al., 2014). Constitutively activated KRAS provokes sustained stimulation of downstream signaling effectors, leading to increased proliferation, suppression of apoptosis, altered metabolism, evasion of immune response, alterations in composition of tumor microenvironment, and metastasis (Pylayeva-Gupta et al., 2011). Thus, oncogenic KRAS activation is a crucial driver of many hallmarks of cancer. 


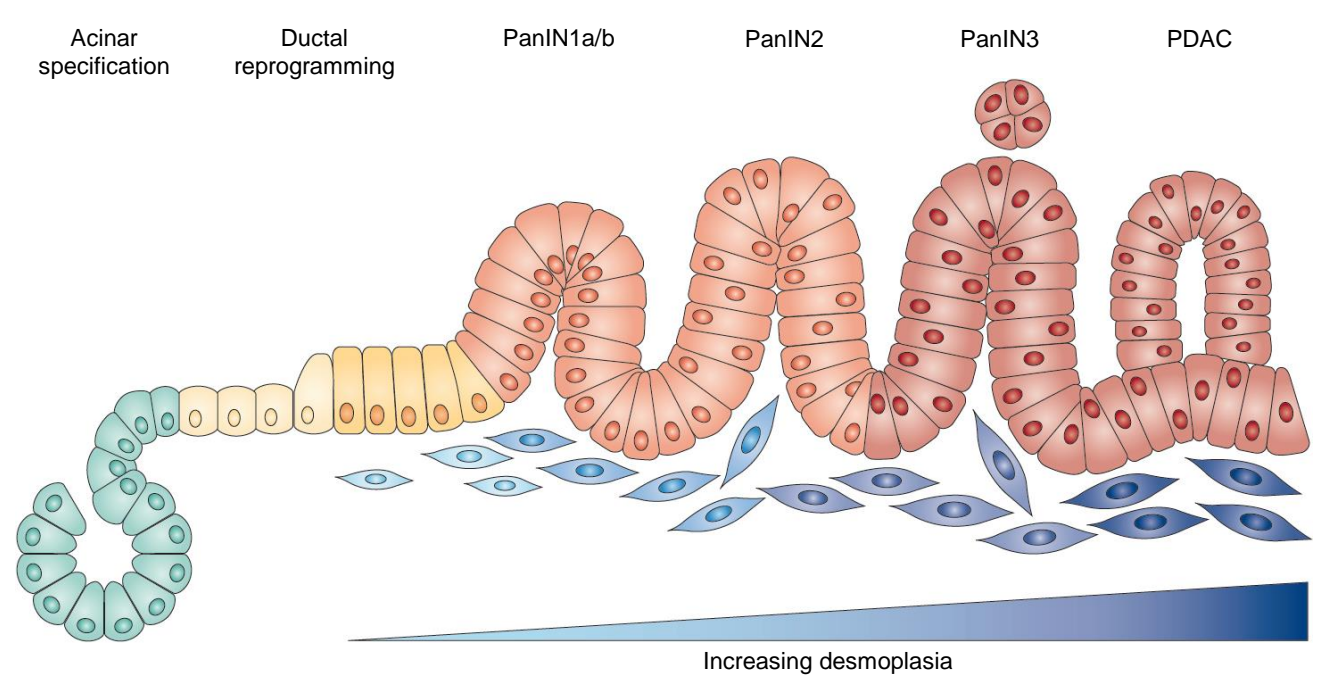

Figure 1 PDAC arises from preneoplastic lesions. The exocrine part of the pancreas is composed of acinar cells having the potential to undergo ductal reprogramming and thus giving rise to PanIN lesions. PanINs are graded with respect to nuclear atypia and loss of cell polarity into four stages. Robust desmoplastic response accompanying PanIN progression is a hallmark of PDAC. Modified from Morris et al. (2010).

PDAC originates from preneoplastic lesions [Figure 1] that are formed in regenerative response to inflammatory insults like acinar-to-ductal metaplasia (ADM) or due to tumor-promoting mutations like oncogenic activation of Kras in pancreatic intraepithelial neoplasias (PanINs) (De La O et al., 2008; Reichert \& Rustgi, 2011). The term $\mathrm{ADM}$ describes an early precancerous lesion originating from the acinar compartment of the pancreas undergoing a potentially reversible transdifferentiation into ductal cells (Reichert \& Rustgi, 2011). Importantly, ADM potentially evolves into PanIN lesions and thereby lose their regenerative capacity (Song et al., 1999; Means et al., 2005; Zhu et al., 2007). Upon their degree of dysplasia, PanIN lesions are categorized into four stages: PanIN1a, PanIN1b, PanIN2, and PanIN3. While PanIN1a starts with minimal morphological alterations, the grade of nuclear atypia and loss of cell polarity increases during PanIN progression towards carcinoma in situ at PanIN3 stage complemented by pleomorphism and nuclear enlargement (Hingorani et al., 2003; Ottenhof et al., 2011). However, although PanIN lesions have the potential to develop into carcinoma, their progression is limited and needs further trigger factors besides oncogenic Kras activa- 
tion to form full-blown, invasive PDAC. Morris et al. (2010) summarized pancreatic carcinogenesis being dependent on accumulative mutations, loss of tumor suppressor genes, a chronic inflammatory environment, and the activation of oncogenic pathways.

Multiple studies demonstrated that oncogenic Kras activation is necessary for PDAC formation, but not sufficient to drive its further development (Hingorani et al., 2005; Guerra et al., 2007; Ardito et al., 2012; di Magliano \& Logsdon, 2013). Besides oncogenic Kras activation, there are a few frequent mutations occurring in PDAC concerning CDKN2A inactivation (encoding for the tumor suppressors p16 $6^{\mathrm{INK} 4 \mathrm{a}}$ and $\mathrm{p} 19^{\mathrm{ARF}}$ ), TP53 mutation potentially leading to gain-of-function of the respective p53 protein, and loss of deleted in pancreatic carcinoma, locus 4 (DPC4), also known as SMAD4. However, Jones et al. (2008) identified an average of 63 genetic alterations in human PDAC, accentuating the vast extent of PDAC heterogeneity. Importantly, PDAC diversity is further enhanced by alterations in the tumor microenvironment like immune cell invasion/evasion and extracellular matrix (ECM) composition as well as tumor cell metabolism, respectively (Carr \& Fernandez-Zapico, 2016; Ying et al., 2016).

Molecular taxonomy of PDAC based on high-throughput genomic and transcriptomic analyses aims to define patient subgroups for precision medicine-based therapeutic implications (Singh et al., 2019). The first attempt of molecular subtyping in PDAC was realized by Collisson et al. (2011), identifying three epithelial subtypes. Moffitt et al. (2015) partly confirmed their data by dissecting two epithelial and two stromal subtypes, respectively. Based on the identification of four epithelial subtypes by Bailey et al. (2016), Maurer et al. (2019) lately reanalyzed patient cohorts and determined in agreement with Moffitt et al. (2015) the classical and basal-like epithelial tumor cell subtypes as well as immune-rich (normal) and ECM-rich (activated) stroma. Eventually, PDAC patient stratification according to their molecular subtypes not only offers prognostic value but also aims at enhancing therapy response.

To the present day, pancreatic surgery remains the only potentially curative treatment option for PDAC. However, its application is limited due to late diagnosis in advanced 
disease stages and early metastatic spread (Adamska et al., 2017). Since 1997, unresectable PDAC is treated with Gemcitabine (2',2'-difluoro 2'-deoxycytidine or dFdC), a nucleoside analog that is still in use for metastatic pancreatic cancer in patients with low performance status (Burris et al., 1997; Ellenrieder et al., 2016). As PDAC is highly refractory to systemic therapies, clinical studies aim at complementing Gemcitabinebased chemotherapy with additional drugs to convey higher specificity and improve patient outcome (Kleeff et al., 2016). In terms of palliative treatment, nano-formulated albumin-bound paclitaxel combined with Gemcitabine emerged as a more effective alternative to Gemcitabine monotherapy (Von Hoff et al., 2013). FOLFIRINOX (a combination of oxaliplatin, irinotecan, leucovorin, and 5-fluorouracil) is the most promising treatment option with significant improvement of life expectancy, but severe side effects restrict its applicability to patients with good performance status (Conroy et al., 2011). Interestingly, Aung et al. (2018) demonstrated that the classical epithelial tumor cell type is associated with good response to first-line chemotherapy, while the basal-like type poorly responded to treatment. Consequently, PDAC patient stratification can offer insights into potential chemotherapeutic responses, elucidating elevated chemoresistance or chemosensitivity towards specific drug combinations that only develop their full potential in one specific subtype. Thus, future precision medicine approaches aim at connecting molecular data from PDAC specimen with intrinsic chemotherapeutic vulnerabilities in order to offer the most promising treatment option to the corresponding patient subgroup.

\subsection{NFATc1 as an inflammatory driver of pancreatic carcinogenesis}

One hallmark of PDAC is the establishment of a chronic inflammatory environment, both favoring cancer formation and progression (Morris et al., 2010). Since Buchholz et al. (2006) introduced the nuclear factor of activated T cells (NFAT) family of inflam- 
matory transcription factors as crucial drivers of PDAC progression, NFAT research has been focusing on scrutinizing the role of respective family members in shaping the transcriptional output of pancreatic cells (Baumgart et al., 2012; Awla et al., 2012; Baumgart et al., 2014; Hessmann et al., 2016). Of the five NFAT family members, nuclear factor of activated T cells, cytoplasmic 1 (NFATc1) plays an outstanding role in providing growth advantage, apoptosis evasion and dedifferentiation to PDAC cells (Baumgart et al., 2014; Chen et al., 2015; Singh et al., 2015; Chen et al., 2017; Hasselluhn et al., in revision). Thus, promotion of NFATc1 signaling during pancreatic carcinogenesis complements oncogenic Kras activation in driving PDAC progression and metastasis.

The activation of NFAT family members is mediated by the $\mathrm{Ca}^{2+} /$ calcineurin signaling pathway (Clipstone \& Crabtree, 1992). Resting highly phosphorylated in the cytoplasm in their inactivated state, NFAT transcription factors can be activated by diverse signaling pathways causing influx of intracellular calcium ions. High calcium concentrations activate calcineurin, which in turn dephosphorylates multiple phosphoserines on NFAT proteins, thereby demasking the nuclear localization sequence (NLS) resulting in nuclear shuttling (Loh et al., 1996; Shibasaki et al., 1996; Garcia-Cozar et al., 1998). In the nucleus, NFAT transcription factors share the consensus site 5'-GGAAA-3' which is preferably located in promoter/enhancer regions of many immune response and proliferation genes (Buchholz et al., 2006). Continuous calcineurin activity is required for sustained NFAT nuclear localization as constitutively active kinases target NFAT for rephosphorylation and subsequent transfer out of the nucleus (Neal \& Clipstone, 2001; Sheridan et al., 2002). Importantly, it is known that NFAT proteins regulate gene transcription insufficiently alone, therefore they rather form complexes with additional transcription factors (Loh et al., 1996; Chen et al., 1998; Baumgart et al., 2014). The transcriptional output of NFAT proteins is highly dynamic and context-dependent and thus can be shaped via respective binding partners. 
For the investigation of NFATc1-dependent processes in PDAC development, Baumgart et al. (2014) developed the cnNFATc1; Kras ${ }^{G 12 D}$; p48-Cre (NKC p48) mouse model. Constitutive nuclear NFATc1 signal in the context of oncogenic Kras activation dramatically accelerates PanIN formation and progression towards PDAC in this genetically engineered mouse model (GEMM) (Baumgart et al., 2014; Hasselluhn et al., in revision). In contrast, pancreas-specific loss of NFATc1 (NFATc1 $\left.1^{\mathrm{A} / \mathrm{fl}} ; \mathrm{Kras}^{\mathrm{G12D}}\right)$ decelerates preneoplastic lesion formation and PDAC establishment compared to NFATc1 wildtype (WT) mice despite oncogenic Kras activation (Baumgart et al., 2014; Chen et al., 2015; Hasselluhn et al., in revision). Additionally, NFATc1 as a malignant promoter of PDAC was highlighted by Singh et al. (2015), demonstrating that NFATc1mediated transcription cooperates with mutation of the tumor suppressor Tp53 in driving PDAC dedifferentiation and metastasis. These findings are in agreement with Diaferia et al. (2016) linking strong NFATc1 expression to high-grade PDAC in human tissue.

\subsection{TGF $\beta$ signaling in PDAC: a double-edged sword}

Robust desmoplastic response in collaboration with immune cell infiltration is one crucial driver of tumor growth, disease progression and drug resistance (Kalluri \& Zeisberg, 2006; Aran et al., 2015). As the stromal compartment comprises up to $90 \%$ of the tumor burden in PDAC (Neesse et al., 2011), understanding of the interaction between epithelial tumor cells and the tumor stroma is crucial for the development of effective cancer treatment strategies. The interplay between diverse cell types in PDAC is based on a multitude of secreted signaling molecules of which transforming growth factor beta $(\mathrm{TGF} \beta)$ is one of the most abundant and complex mediators. TGF $\beta$ is produced and released by fibroblasts, endothelial cells, macrophages, and lymphocytes (Oft et al., 1996) and its influence propagates through all cell types that contribute to the tumor composition. 


\subsubsection{Canonical and non-canonical TGF $\beta$ pathway}

According to their ligands, the TGF $\beta$ family subdivides into TGF $\beta$, Activin, and bone morphogenetic protein (BMP) subfamilies (Heldin et al., 1997) [Figure 2 A]. The TGF $\beta$ group is unique in its receptors (TGF $\beta$ receptor-I/II (T $\beta$ R-I/II)) but shares downstream Smad proteins with the Activin group (Heldin et al., 1997). Smad2 and Smad3 proteins are activated upon receptor-mediated phosphorylation and form complexes with the common-Smad (Smad4) prior to translocation to the nucleus and subsequent activation of transcription (Eppert et al., 1996; Zhang et al., 1996; Nakao et al., 1997b). In contrast, BMP signaling is mediated by receptor-phosphorylation of Smad1, Smad5, and Smad8 proteins forming hetero-oligomers with Smad4 for subsequent gene

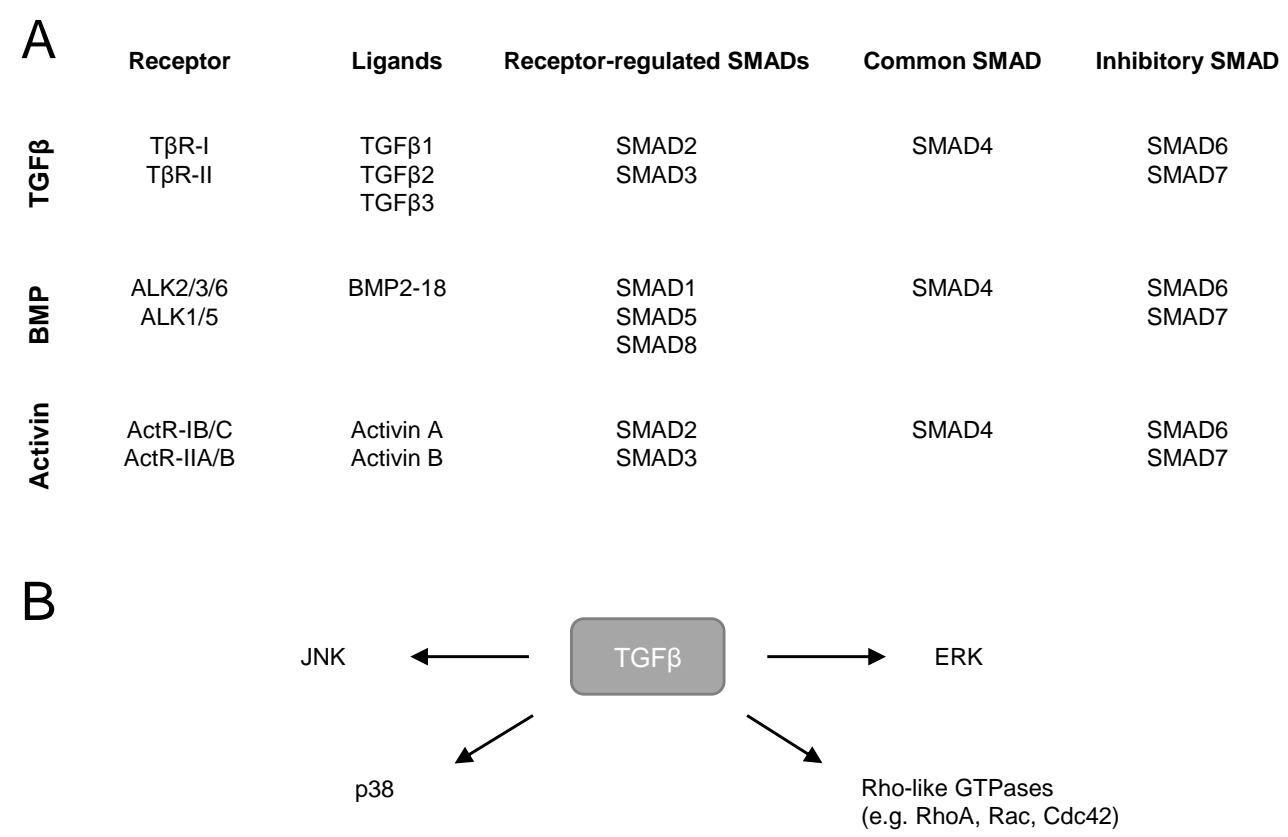

Figure 2 Canonical and non-canonical TGF $\beta$ signaling. A The TGF $\beta$ superfamily comprises Activin, BMP, and TGF $\beta$ canonical signaling cascades according to their respective ligand. The common SMAD binding partner SMAD4 and the inhibitory SMADs (SMAD6/7) act throughout the superfamily. B Non-canonical TGF $\beta$ signaling is mediated via receptor activation but the exact way of signal transduction to effector proteins remains elusive. Only the best-characterized targets are shown. Modified from Heldin et al. (1997); Derynck \& Zhang (2003); Rahman et al. (2015). 
regulation (Attisano \& Wrana, 2000). The inhibitory Smads (Smad6 and Smad7, respectively) exert their function throughout the TGF $\beta$ family and disrupt signaling by binding to type I receptors, interfering with phosphorylation of pathway-restricted Smads (Imamura et al., 1997; Nakao et al., 1997a). As the transcription of inhibitory Smads is induced by TGF $\beta$ itself, Heldin et al. (1997) suggest inhibitory Smads acting as autoregulatory negative feedback loops in the signal transduction of the TGF $\beta$ superfamily. In addition, the non-canonical TGF $\beta$ signaling comprises the activation of the Ras signaling cascade (Derynck \& Zhang, 2003).

Despite their significant homology in sequence and structure, Smad2 and Smad3 exert fundamentally different functional roles in TGF $\beta$ signaling (Yang et al., 2003). According to studies in mouse embryonic fibroblasts by Yang et al. (2003), Smad3 deficiency completely blocks TGF $\beta$ response. In contrast to Smad2, Smad3 and Smad4 directly bind DNA at the palindromic sequence 5'-GTCTAGAC-3' known as the Smad binding element (SBE) (Zawel et al., 1998). Smad3 primarily activates transcriptional regulators to initiate a cascade of secondary gene regulation (Yang et al., 2003). Importantly, TGF $\beta$-regulated intermediate-early genes are highly cell type-dependent (Yang et al., 2003) which is in agreement with the strong role of Smad binding partners in defining target gene and pathway specificity as well as the transcriptional effect (Massagué et al., 2005). For sufficient TGF $\beta$ activation, several SBE copies are required while binding variability is mediated by positions outside the SBE (Zawel et al., 1998). Due to its known synergism with the RAS cascade and activator protein 1 (AP-1) transcription factors, de Caestecker et al. (2000) propose SMAD3 as a key mediator of oncogenic TGF $\beta$ signaling.

The TGF $\beta$ family of cytokines is responsible for a plethora of developmental and homeostatic processes as it unites a multitude of different signaling cascades that can be dysregulated in disease. Cellular responses include initiation of cell cycle arrest, apoptosis, differentiation, cell motility, extracellular matrix production, angiogenesis, and cellular immune response (Zawel et al., 1998; Derynck \& Zhang, 2003). In early 
disease stages and in healthy tissue, TGF $\beta$ acts as a tumor suppressor by exerting antimitogenic effects (Heldin et al., 1997) mediated by Cyclin-dependent kinases (Matsuura et al., 2004) in addition to promotion of cytostasis or apoptosis (Massagué, 2008). However, during cancer development, cells acquire resistance towards the growth inhibiting functions of TGF $\beta$ and exploit its full potential in a tumor-promoting manner (Massagué, 2008; Ikushima \& Miyazono, 2010). Accordingly, detected levels of TGF $\beta$ in carcinomas exceed those observed in corresponding healthy tissue by far (Pardali \& Moustakas, 2007). Importantly, altered TGF $\beta$ signaling is one hallmark of PDAC, including the dysregulation of both canonical and non-canonical pathway (Bailey et al., 2016). Oncogenic TGF $\beta$ actions comprise fundamental changes in stroma composition, evasion of immune response, angiogenesis, and tumor cell invasiveness (Pardali \& Moustakas, 2007).

Until now, the contextual mechanism how the switch from tumor-suppressive to tumorpromoting TGF $\beta$ signaling is conveyed is still a matter of debate. For pancreatic cancer, the critical involvement of Krüppel-like factor 10 (KLF10), NFATc1, and SMAD4, have been discussed in the context of oncogenic KRAS activation (Mishra et al., 2017; Singh et al., 2010; Zhang et al., 1997). SMAD4 deletion concerns $55 \%$ of all patients, characterizing it as one of the most frequent genetic events in PDAC (Hahn et al., 1996a,b; Wilentz et al., 2000a). Homozygous inactivation of both alleles results in complete loss of function (Schutte et al., 1996), thus featuring aggressive PDAC and metastasis formation in patients (Shin et al., 2017). Importantly, SMAD4 deficiency occurs at the stage of established PDAC, while its expression levels remain intact during PanIN progression (Wilentz et al., 2000a; Hosoda et al., 2017). As there is no progressive reduction of SMAD4 expression, immunohistochemistry (IHC) serves as an excellent tool to detect SMAD4 deactivation, regardless of its inactivation mechanism (Wilentz et al., 2000a). Loss of Smad4 is associated with TGF $\beta$-mediated epithelialmesenchymal transition (EMT), a well-coordinated process during embryonic development and wound healing which turns to a pathological feature in neoplasia and fibrosis (Duda et al., 2003; Thiery, 2003; Subramanian et al., 2004; Massagué, 2008). 
Cells undergoing EMT gradually lose expression of epithelial cell junctions in favor of a more mesenchymal cytoskeleton leading to increased cell motility (Massagué, 2008) and resistance to growth inhibition by TGF $\beta$ (Oft et al., 1996). In addition, Jazag et al. (2005) associated Smad4 depletion in PDAC with decreased cell adhesion and increased cell motility. Thus, induction of EMT by loss of Smad4 contributes to tumor progression and metastasis formation by promoting invasion and dissemination of motile cancer cells (Mani et al., 2008).

TGF $\beta$ utilizes various intracellular signaling pathways in addition to SMADs to regulate a wide array of cellular functions via non-canonical signaling (Zhang, 2009) [Figure 2 B]. By direct ligand-occupied receptor activation, the TGF $\beta$ signaling cascade interacts with a multitude of mitogen-activated protein kinases (MAPKs) such as extracellular signal-regulated kinase (ERK), cJun N-terminal kinase (JNK) and p38 kinases (Hartsough \& Mulder, 1995; Engel et al., 1999; Hanafusa et al., 1999; Hocevar et al., 1999; Sano et al., 1999; Bhowmick et al., 2001; Yu et al., 2002; Zhang, 2009). Yang et al. (2003) demonstrated that ERK function is required for efficient activation of a certain gene set, while other genes depend on ERK inactivation to be susceptible for TGF $\beta$-induced regulation. Importantly, it was evidenced that ERK signaling enhances SMAD3-dependent gene activation by positive interaction with activated SMADs (de Caestecker et al., 1998; Zhang et al., 1998). JNK and p38 have been implicated in TGF $\beta$-induced apoptosis, EMT, cell motility, and cancer cell invasion (Bhowmick et al., 2001; Yu et al., 2002). Therefore, the broad spectrum of regulated genes in combination with its huge potential to interact with additional pathways and its role in mediating signals from tumor to stromal cells establishes TGF $\beta$ pathway as one of the most important signaling cascades in PDAC. 


\subsubsection{Cooperativity of NFAT and TGF $\beta$ signaling cascades}

NFATc1 does not only exert its oncogenic potential in the epithelial tumor cells, but establishes an inflammatory microenvironment promoting further tumor progression (Baumgart et al., 2014). Singh et al. (2010) demonstrated that NFATc1 signaling is closely linked to TGF $\beta$ activation as TGF $\beta$ induces NFATc1 expression and activation as a mechanism to overcome TGF $\beta$-mediated growth arrest. However, in the setting of constitutively active NFATc1 signaling, TGF $\beta$ counteracts NFATc1-mediated proliferation and apoptosis evasion (Hasselluhn et al., in revision). The strong PDAC plasticity and interconnection of a plethora of signaling pathways result in a high degree of context dependency emphasizing the need to comprehend underlying molecular patterns to successfully counteract oncogenic gene signatures.

As both SMAD3 and NFAT transcription factors are implicated in shaping the tumor microenvironment, functional cooperativity of both transcription factors was investigated in diverse cellular systems (Tone et al., 2008; Singh et al., 2010; Tardif et al., 2013; Sengupta et al., 2013; Mokrani et al., 2014). First, NFAT and Smad3 cooperativity was described in immune cells. Tone et al. (2008) identified SMAD3 cooperating with NFAT at the FOXP3 enhancer and mediate histone acetylation with subsequent FOXP3 protein induction in T regulatory cells. In addition, Mokrani et al. (2014) showed SMAD2/3 and NFAT1 coregulation of CD103 expression on T lymphocytes contributing to cytotoxic t cell response and cancer cell destruction. In contrast, Tardif et al. (2013) demonstrated micro RNA-140 (miR-140) regulation by NFAT3 as a transcriptional activator as opposed to SMAD3 repressor activity. This interplay is in agreement with Singh et al. (2010), elucidating NFAT competing with SMAD3 in regulation of MYC protooncogene in PDAC. On a functional level, Sengupta et al. (2013) identified NFAT silencing as efficient inhibitor of TGF $\beta$-mediated EMT in breast cancer and suggested NFAT/SMAD3 complex formation. However, direct transcription complex formation of NFAT and SMAD proteins has not been shown in any cellular system yet and thus remains a matter of investigation. 


\subsection{Mechanisms behind Kras-driven PDAC}

Oncogenic Kras activation occurs in over $90 \%$ of PDAC patients, making it the most prominent driving force in pancreatic carcinogenesis initiation (Hingorani et al., 2003). Kras belongs to the family of Ras proteins that share approximately $80 \%$ sequence homology and are distinguished by their carboxy-terminal hypervariable regions (Nussinov et al., 2015). Ras proteins are ubiquitously expressed and involved in the regulation of cell proliferation, differentiation, survival, migration, and apoptosis (Nussinov et al., 2015). They bind guanosine triphosphate (GTP) and anchor in the membrane for their activation and subsequent signaling (Nussinov et al., 2017). In turn, GTPase-activating protein mediates GTP to guanosine diphosphate (GDP) hydrolysis, a process that is reversible by exchanging GDP by GTP with the help of guanine nucleotide exchange factor (Nussinov et al., 2017). Importantly, for oncogenic Ras, steric hindrance impedes with hydrolysis of GTP by GTPase-activating protein, thus resulting in constitutive Ras signaling independent of extracellular activation signals (Schlichting et al., 1990; Scheffzek et al., 1997; Wittinghofer \& Vetter, 2011).

However, the complex and dynamic Kras signaling cascades are still not entirely understood. Nussinov et al. (2015) elucidate oncogenic Kras signaling, disclosing strong redundancy of pathways, temporality of cell decisions and variation of preferred signaling cascades in different tumor entities. In PDAC, there are four major pathways involved: RAF/mitogen-activated protein kinase kinase (MEK)/ERK, phosphatidylinositol 3 kinase (PI3K)/3-phosphoinositide-dependent protein kinase 1 (PDK1)/RAC-alpha serine/threonine-protein kinase (AKT), JNK, and guanine nucleotide exchange factor (Lim et al., 2005; Feldmann et al., 2010; Collisson et al., 2012; Eser et al., 2013, 2014; Davies et al., 2014). While Eser et al. (2013) and Eser et al. (2014) highlighted the pivotal role of PI3K/PDK1/AKT cascade for ADM and PanIN formation as well as PDAC establishment, Collisson et al. (2012) described $\mathrm{RAF} / \mathrm{MEK} / \mathrm{ERK}$ signaling as a driver of neoplastic alterations, indicating that both pathways are crucial in PDAC initiation and development. In contrast, impaired JNK 
signaling in the context of oncogenic KRAS activation was associated with acceleration of PDAC progression and reduced regenerative capacity of acini (Davies et al., 2014). Meanwhile, Lim et al. (2006) and Vigil et al. (2010) discovered strong activation of guanine nucleotide exchange factors in PDAC. However, it remains unclear how oncogenic Kras activation signals to preferred context-dependent downstream cascades during carcinogenesis and how the complex network interacts.

An important nuclear target of the RAF/MEK/ERK cascade is the transcription factor family AP-1 (Johnson et al., 1996). Lamph et al. (1988) and Johnson et al. (1996) described AP-1 activation relying on the same stimuli as ERKs, including oncogenic RAS and growth factors. The AP-1 family comprises heterodimers and homodimers of JUN, FOS, MAF and ATF transcription factors known for their crucial role in mediating cell proliferation, survival and cell death (Ryseck et al., 1988; Shaulian \& Karin, 2001). One crucial mediator of the JUN transcription factors is cJUN (Bohmann et al., 1987), whose homozygous depletion leads to severe impairment of AP-1 transcriptional response and prevents transformation by RAS (Johnson et al., 1996). Importantly, cJUN activity is controlled by JNK-mediated phosphorylation and dominant negative cJUN alleles lacking the JNK phosphorylation sites impede with RAS transformation (Lloyd et al., 1991; Smeal et al., 1991). Conversely, RAS activation triggers cJUN expression, promotes AP-1 binding activity and thus determines its full oncogenic potential (Johnson et al., 1996). In contrast, Johnson et al. (1996) demonstrated that mutated RAS does not signal through induction of JUNB and JUND, respectively, thus highlighting the particular importance of cJUN as a downstream effector of oncogenic $R A S$ activation.

Kras signaling is strongly intertwined with the TGF $\beta$ signaling pathway. First of all, Oft et al. (1996) demonstrated RAS mutation promoting TGF $\beta$ mRNA and protein expression in mammary epithelial cancer cells. In this autocrine manner, constant activation of the TGF $\beta$ cascade is ensured. Importantly, RAS-transformed cells lose capacity to respond to TGF $\beta$ with growth arrest (Kretzschmar et al., 1999; Sekimoto et al., 
2007), resulting in diminished tumor-suppressive TGF $\beta$ activity without abrogation of TGF $\beta$ responsiveness. In contrast, cells may respond with invasive and metastatic behavior (EMT) instead (Kretzschmar et al., 1999). This alteration in downstream regulated gene signature can be mediated via SMAD3 linker domain phosphorylation by KRAS-driven MAPKs such as ERK, JNK, and p38 (Kretzschmar et al., 1999; Furukawa et al., 2003; Mori et al., 2004; Sekimoto et al., 2007). This is in agreement with altered TGF $\beta$ responsiveness in RAS-transformed cells (Sekimoto et al., 2007; Hasselluhn et al., in revision), presenting tumor-promoting TGF $\beta$ signaling as a functional collaboration of oncogenic RAS activation and SMAD proteins. Interestingly, the AP-1 complex integrates signaling from both TGF $\beta$ and MAPK pathways (Liberati et al., 1999). Moreover, KRAS mutation interferes with SMAD4-dependent senescence induction by TGF $\beta$, thus making loss of SMAD4 superfluous for early PanIN development (Fullerton et al., 2015). However, Leung et al. (2013) claim that malignant transformation of normal ductal pancreas cells requires KRAS mutation and loss of SMAD4 to overcome TGF $\beta$-mediated growth arrest. In the context of SMAD4 deficiency in PDAC, oncogenic KRAS and activated TGF $\beta$ signaling synergize in driving oncogenic gene signatures to their full potential.

\subsection{Aims of the study}

PDAC is a highly lethal disease characterized by high metastatic spread, chemoresistance, and its huge genetic heterogeneity. The most prominent genetic alteration is the oncogenic activation of Kras in $90 \%$ of all PDAC patients, initiating early preneoplastic lesion formation in the pancreas which eventually develops into carcinoma stage. Loss of Smad4 is a frequent event in established PDAC (55\%) and is of predictive value concerning metastasis formation, thus making it an extensively studied subject over the last decades. 


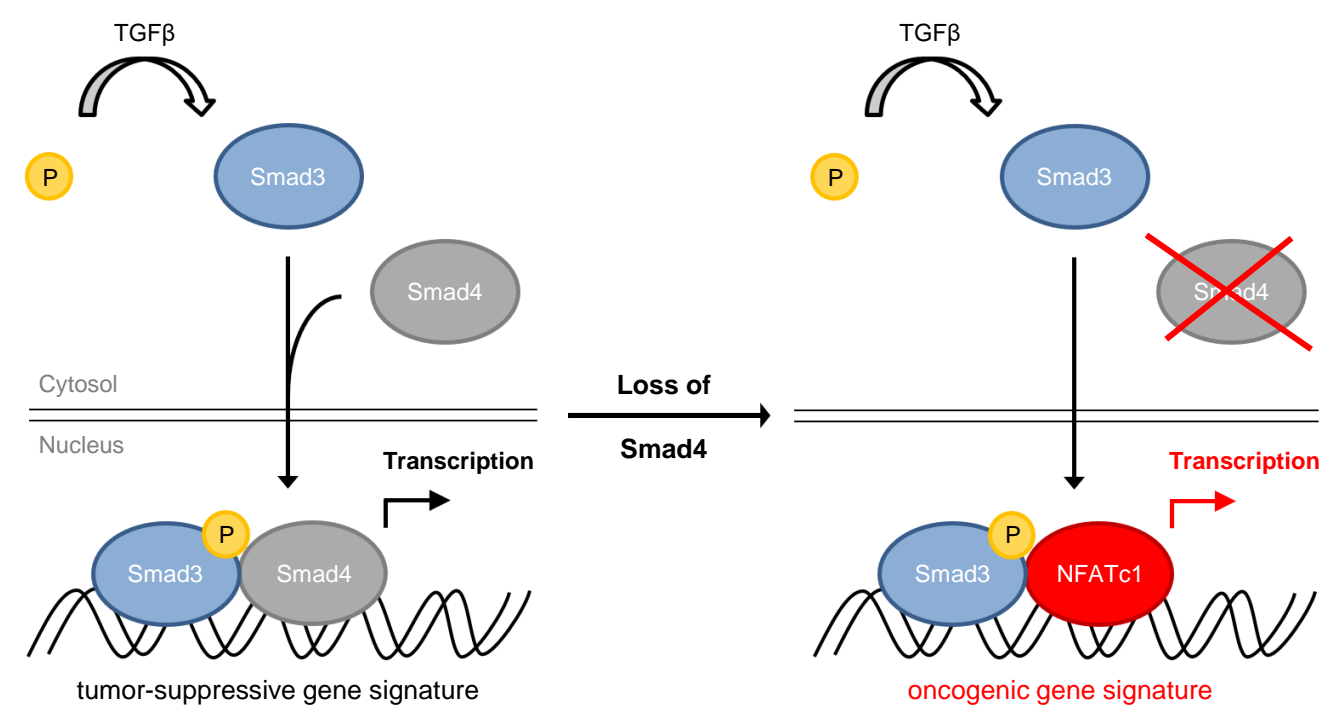

Figure 3 NFATc1 as an alternative Smad3 binding partner in Smad4deficient PDAC. Loss of Smad4 is a frequent event in PDAC progression and a known mediator of the switch from tumor-suppressive to oncogenic gene signatures. We hereby propose that alternative aggressive gene signatures are enforced by NFATc1/Smad3 transcription complexes, exploiting tumor-promoting signatures in TGF $\beta$-driven PDAC.

Smad4 is a crucial part of the TGF $\beta$ signaling cascade, mediating the translocation of activated receptor Smads into the nucleus. However, in the absence of $\operatorname{Smad} 4$, TGF $\beta$ signaling is not abrogated, but comprises alternative signaling pathways resulting in the activation of differential transcription programs. Loss of Smad4 is implicated in driving oncogenic TGF $\beta$ signatures towards more invasive and aggressive PDAC. However, the exact mechanism how Smad3-mediated transcription in the context of Smad4 deficiency is hijacked in the direction of tumor-promoting gene transcription remains elusive. Here, we aim to study the mechanistic and functional consequences of Smad4 deficiency in PDAC on oncogenic Smad3 transcription complex formation and gene transcription. We focus our study on NFATc1, an inflammatory transcription factor implicated in PDAC development and progression. Previous work from our laboratory already suggested functional cooperativity between Smad3 and NFATc1, which is further exploited in this project. 
We hereby propose that the formation of NFATc1/Smad3 transcription complexes is required for TGF $\beta$-promoted cancer cell plasticity in a Smad4-deficient PDAC subtype [Figure 3]. Based on this hypothesis, this study aims to (i) determine if NFATc1 is an alternative Smad3 binding partner in Smad4-depleted PDAC, (ii) elucidate permissive conditions for proposed transcription complex assembly, (iii) identify gene signatures regulated by NFATc1/Smad3 complexes, and (iv) develop a strategy on how to disrupt respective transcription complexes in favor of reestablishment of tumor-suppressive TGF $\beta$ signaling. By combining in vivo and in vitro approaches, we aim to scrutinize whether targeting of potential NFATc1/Smad3 complexes overcomes tumor-progressive functions of Smad4 deficiency and therefore represents a promising pharmacological strategy to tackle Smad4-depleted PDAC. 


\section{Material and Methods}

In this chapter, material and methods are described which were crucial in data acquisition and analysis for this thesis. However, some studies were conducted as part of cooperations, so detailed protocols are not described here. Dr. med. H. Bohnenberger, Institute of Pathology, University Medical Center Göttingen provided human tissue microarrays (TMAs), assisting in staining and analysis. Moreover, he enhanced our tumor studies by offering Masson's trichrome staining for tumor-bearing mice. In the meantime, tumor grading of respective PDAC-bearing mice was kindly conducted by Prof. P. Ströbel, Institute of Pathology, University Medical Center Göttingen. In an exchange with Prof. S. Hahn, Clinical Research Center, Ruhr-Universität Bochum and Prof. J. Siveke, West German Cancer Center, University Hospital Essen, we retrieved primary human PDAC cells gathered from patient-derived xenograft (PDX) studies. Sequencing of respective PDX-derived cells was conducted by Prof. B. Wollnik and PD Dr. rer. nat. S. Kaulfuß, Department of Human Genetics, University Medical Center Göttingen. Additionally, Dr. S. Nagarajan, Cambridge Research Institute, University of Cambridge, enhanced our studies by comparison of published chromatin immunoprecipitation sequencing (ChIPseq) data.

Graphs were visualized using GraphPad Prism (version 8.0.1). Data are presented as mean \pm standard deviation. Significance was tested by Student's t-test if not stated otherwise, presenting significance as ${ }^{*} \mathrm{p}<0.05$, ** $\mathrm{p}<0.01$, and $* * * \mathrm{p}<0.001$, respectively. 


\subsection{Cell culture}

\subsubsection{PDAC cells and cultivation}

A variation of PDAC cell lines was used to comprehend Smad4-dependent processes in vitro. NKC-II cells are primary murine PDAC cells derived from a tumor-bearing cnNFATc1; Kras ${ }^{G 12 D}$; p48-Cre (NKC p48) mice. Its establishment is described by Baumgart et al. (2014). Accordingly, murine KPC Bl6 cells are derived from the corresponding Kras ${ }^{G 12 D}$; $p 53^{R 172 C /+}$; pdx-Cre mouse model and were gifted by PD Dr. Dr. med. A. Neeße. Importantly, KPC mice were bred with a pure C57BL/6 background making this cell line applicable for orthotopic transplantation experiments in C57BL/6-J mice. Sequencing of TP53 locus conducted by L. Versemann, Department of Gastroenterology and Gastrointestinal Oncology, University Medical Center Göttingen, dissected the R172C transition instead of the expected $\mathrm{R} 172 \mathrm{H}$ alteration. Clones derived from NKC-II or KPC Bl6 cells were cultivated in Dulbecco's modified Eagle's medium (DMEM) (Gibco, 41 965-62) supplemented with $10 \%$ fetal bovine serum (FBS) (Biowest, S181B-500) and 1\% non-essential amino acids (NEAA). In contrast, human cell lines PaTu8988t and Bo62 were maintained in DMEM supplemented with $10 \%$ FBS. Human BxPC3 cells are grown in Roswell Park Memorial Institute (RPMI) (Thermo Fisher, 61870044 ) enriched with 10\% FBS and 1\% L-Glutamine.

In general, cells were cultivated at $37^{\circ} \mathrm{C}$ and $5 \% \mathrm{CO}_{2}$ until they reached $90 \%$ confluency. For subcultivation, the medium was removed, cells were washed with phosphate buffered saline (PBS) followed by trypsinization (Gibco, 15 400-54; 1:10 dilution in PBS). Trypsinization was stopped by adding cultivation medium and cells were seeded according to the respective experiments. 


\subsubsection{Treatment and transfection}

Cells were allowed to attach overnight $(\mathrm{o} / \mathrm{n})$ prior to further treatment. For TGF $\beta$ (PeproTech, 100-21B, 10 ng/ml) and a selection of Trametinib (Biomol, Cay16 292-50) studies, cells were serum-starved in their respective medium for $24 \mathrm{~h}$ prior to treatment for 24 h. 1,4-diamino-2,3-dicyano-1, 4-bis[2-aminophenylthio]butadiene (UO126) (Calbiochem, 662 005) was added for $24 \mathrm{~h}$ in normal cultivation medium. In contrast, studies with Gemcitabine-Hydrochloride (Sigma-Aldrich, G6423) were incubated for either $48 \mathrm{~h}$ or $72 \mathrm{~h}$ and cytosine arabinoside (AraC) for $72 \mathrm{~h}$ (Sigma-Aldrich, C1768). As Trametinib and UO126 are dissolved in dimethyl sulfoxide (DMSO), sole DMSO treatment served as treatment control (ctrl).

AraC, and UO126 were kindly provided by Prof. M. Dobbelstein, Institute of Molecular Oncology, University Medical Center Göttingen and Trametinib was a gift from Prof. S. A. Johnsen, Department of General, Visceral and Pediatric Surgery, University Medical Center Göttingen and Division of Gastroenterology and Hepatology, Department of Internal Medicine, Mayo Clinic, Rochester (Minnesota/USA).

Table 1 Sequences of siRNAs. Gene silencing was performed by transfecting siRNAs by Thermo Scientific using the equivalent volume of siLentFect lipid reagent (Bio-Rad) in OptiMEM (Gibco, 31 985-62).

\begin{tabular}{lcl}
\hline Target & No & Sequence \\
\hline cJun & 155942 & 5'-GCG CAU GAG GAA CCG CAU Utt-3' \\
NFATc1 & 288360 & 5'-GCG UUU CAC GUA CCU UCC Utt-3' \\
Nfatc1 & MSS275982 & 5'-AGG ACA GGA AGU AUC CCG AAG GCC C-3' \\
Smad3 & 156946 & 5'-CGC AGA ACG UGA ACA CCA Att-3' \\
Smad4 & 62739 & 5'-GGA UUU CCU CAU GUG AUC Utt-3' \\
\hline
\end{tabular}

For gene silencing experiments, small interfering RNA (siRNA) specific for respective targets was employed. Sequences used in this thesis are depicted in table 1. In short, the same volume of the designated siRNA and siLentFect ${ }^{\circledR}$ lipid reagent (BioRad) was added to OptiMEM (Gibco, 31985-62) and incubated for $20 \mathrm{~min}$ before adding the solution to the cells. Overexpression studies were conducted with either a 
SMAD3-FLAG (Feng et al., 1998; Zhang et al., 1998) or SMAD4-HA construct (Abdollah et al., 1997) by transfection with Lipofectamine ${ }^{\circledR} 2000$ (invitrogen by Thermo Fisher Scientific, 11668 019). As an exception, PaTu8988t cells were always transfected with TransFast $^{\mathrm{TM}}$ (Promega, E2431), due to extreme sensitivity towards Lipofectamine reagent.

\subsubsection{CRISPR/Cas9-mediated Smad4 knock out}

Using the double nicking CRISPR/Cas9-mediated genome editing approach, Smad4 WT and k.o. clones of murine NKC-II, KPC Bl6 and human Panc-1 PDAC cells were established.

To mediate a Smad4-specific gene knock out, sgRNAs (single guide RNAs) for human and murine Smad4 WT expressing cells were designed, using the tools provided by Ran et al. (2013b) and Xu et al. (2015). For each species, two sgRNAs were designed to provide the excision of the complete exon 3 and to introduce a base shift, guaranteeing Smad4 protein truncation, misfolding and loss of functionality (Ran et al., 2013a). Subsequently, phosphorylated oligomers were ordered (biomers.net) and cloned into the px-458 vector bearing the Cas9 endonuclease, a GFP-tag (green fluorescent protein) and resistance to ampicillin. CRISPR/Cas9 vector was used for E.coli (Escherichia coli) transformation and plated on LB (lysogeny broth) agarose plates supplemented with ampicillin. Correct insertion was confirmed by sequencing, and positive E.coli clones were used to inoculate $100 \mathrm{ml} \mathrm{LB}$ medium in order to generate more vector material for a midi plasmid preparation (Nippon Genetics, FG-90 302). Deoxyribunucleic acid (DNA) concentration was measured by NanoPhotometer P-Class 360 (intas).

PDAC cells were thawed and subcultivated for one passage. Cells were seeded in $10 \mathrm{~cm}$ dishes and maintained until $60 \%$ confluency before transfection with $5 \mu \mathrm{g}$ of each construct in addition to $25 \mathrm{\mu l}$ Lipofectamine ${ }^{\circledR} 2000$ (invitrogen by Thermo Fisher Scientific). Successful transfection was confirmed by GFP fluorescence microscopy. After 
Table 2 sgRNAs designed for CRISPR/Cas9-mediated knock out. sgRNAs were designed using tools provided by Ran et al. (2013b); Xu et al. (2015). Due to a lack of homology between murine and human Smad4, different sgRNAs targeting exon 3 were designed for each species. Knock out strategy involved targeting of introns guiding the respective exon to enable the complete excision of it.

\begin{tabular}{lccl}
\hline Number & Target & Species & Sequence \\
\hline 1 & Nfatc1 & murine & 5'-GCTGTAGCTCGGCACTGCAG-3' \\
2 & Nfatc1 & murine & 5'-TCGTCTCGCAGGCACTGCAG-3' \\
1 & SMAD4 & human & 5'-TCTAGGTCTGATGTATGACA-3' \\
2 & SMAD4 & human & 5'-CGACTTTAAATAAGGTTAAA-3' \\
1 & Smad4 & murine & 5'-TCAGTTAGCTTAGATCTCAG-3' \\
2 & Smad4 & murine & 5'-CCAGTTCCTGAGGCCTGGC-3' \\
\hline
\end{tabular}

$48 \mathrm{~h}$ cultivation, cells were trypsinized and washed twice with PBS. Subsequent to centrifugation (400 x g, $5 \mathrm{~min}$ ), cells were resuspended in $1 \mathrm{ml}$ PBS and filtered through a $50 \mu \mathrm{m}$ cell strainer (BD Biosciences, 340 631) into a FACS tube (special tube used for fluorescence-activated cell sorting; Sarstedt, 55.1579) to generate a single cell suspension. Single cell sorting of highly GFP-positive cells was provided by S. Becker, Department of Hematology and Oncology, University Medical Center Göttingen, using the FACSAriaII cell sorter (BD Biosciences).

After two weeks, emerging cell colonies were marked and strictly observed. If more than one colony formed in the same well, this heterogeneous clone was excluded from further analysis. Upon confluency, cell clones were trypsinized and transferred to a bigger formats. Cells were taken for genomic DNA (QIAGEN; DNeasy Blood \& Tissue Kit, $69504)$, RNA and WCL isolation. Cell stocks of all clones were generated and frozen at $-80^{\circ} \mathrm{C}$ and stored in liquid nitrogen for later use. Promising clones identified by polymerase chain reaction (PCR) with subsequent band separation on an $1 \%$ agarose gel [table 12] were sequenced to prove successful Smad4 depletion and no alterations in potential Smad4 WT clones [see primer sequences in table 3 and general PCR protocol in 2.5.1]. Upon confirmation of Smad4 status, cells were further characterized in treatment studies and functional analyses. 
Note that the design of sgRNAs for CRISPR/Cas9-mediated Nfatc1 k.o. in murine PDAC cells was conducted by myself, while cloning, sorting, expansion of cell clones and characterization was performed by K. Reutlinger, Department of Gastroenterology and Gastrointestinal Oncology, University Medical Center Göttingen.

Table 3 Primer sequences for confirmation of Smad4 knock out. Primers were diluted to $10 \mu \mathrm{M}$ prior to preparation of reaction mix.

\begin{tabular}{lcl}
\hline Target & Direction & Sequence \\
\hline P1 murine & forward & 5'-TGC CAC AGT CTT TGC TGT GA-3' \\
& reverse & 5'-TTC CCA TAC TGT TTG CAA CAA CC-3' \\
P2 murine & forward & 5'-TTC CCT TCA GCA GAA GCT GG-3' \\
& reverse & 5'-GCC ATT TCA ATG TAA AAG TGG GGT-3' \\
P2 human & forward & 5'-TTT ACA CAT AGT TTC TGC AAC ACA GTC-3' \\
P1 human & reverse & 5'-TCT CAA AAA CAA TGT TCT AAA GGG GC-3' \\
\hline
\end{tabular}

\subsection{Functional assays}

\subsubsection{Annexin $\mathrm{V}$ and propidium iodide staining}

Cells were seeded in cultivation medium and attached $\mathrm{o} / \mathrm{n}$. On the next day, cells were treated with indicated concentrations of Gemcitabine (Sigma-Aldrich, G6423) and incubated for $48 \mathrm{~h}$. Then, cells were carefully trypsinized, centrifugated and the supernatant was aspirated. Subsequently, cells were first washed with Annexin V binding buffer (Biolegend, 422 201), transferred to a FACS tube and the volume adjusted to $100 \mu \mathrm{l}$ by decantation. Cells were resuspended in Annexin V binding buffer prior to Annexin V (Biolegend, 640920) and propidium iodide (Sigma-Aldrich, P4864) staining for 15 min. Finally, the volume was adjusted to $300 \mu l$ before measurement at FACS Canto II (BD Biosciences) using FACS Diva Software (version 6.1.3). Data were analyzed by FlowJo software (version 10.1r1) and visualized by GraphPad Prism. 


\subsubsection{Migration assay}

Cells were seeded in 12 well plates and incubated until confluency. Serum starvation $\mathrm{o} / \mathrm{n}$ was followed by introduction of the wound with a $10 \mu \mathrm{l}$ tip. Subsequently, medium was removed and cells were washed with PBS prior to adding serum-free medium. TGF $\beta$ was added directly before starting life cell imaging at Leica microscope (DMi8, CTR advanced), taking one picture per well per hour for $12 \mathrm{~h}$. Migration speed was evaluated by measuring distance (in $\mu \mathrm{m}$ ) of cell borders at three regions per picture and calculate the mean migration speed of 8 sequential pictures. To evaluate the total migration distance, cell borders of the start picture were compared to one taken $5 \mathrm{~h}$ later in the same well in a similar approach.

\subsubsection{MTT assay}

Cell viability and metabolic activity were measured by methylthiazolyldiphenyl tetrazolium bromide (MTT) assay. 2000 cells per well were plated in quintuplicates in 96 well plates in cultivation medium. Treatment scheme including medium change and concentration of substances evaluated in this assay are described in the respective figure legends. On the last treatment day, MTT (Sigma, M5655, $5 \mathrm{mg} / \mathrm{ml}$ in aqua destillata (Aqua dest.)) was added 1:10 into each well, followed by $2 \mathrm{~h}$ incubation. Subsequently, the supernatant was aspirated and cells were disrupted using solubilization solution (100 mM HCl and 10\% Triton X-100 in isopropyl alcohol) by shaking for $20 \mathrm{~min}$ at RT. Measurements were taken at $595 \mathrm{~nm}$ wavelength using a photometer (Autobio, Phomo). Data were processed by subtracting the background and normalizing to treatment control prior to visualization.

For half maximal inhibitory concentration $\left(\mathrm{IC}_{50}\right)$ evaluation, GraphPad Prism software was used, utilizing the logarithmic transformation with subsequent nonlinear regression analysis with sigmoidal shape, defining the end and start values. 


\subsubsection{Cell counting assay}

Cell counting assay was started by plating 20000 cells per well in triplicates in a 6 well plate. Every second day, cells were trypsinized and counted prior to replating in a fresh 6 well plate. Note that the format changed in the last incubation step to a $10 \mathrm{~cm}$ dish to allow unrestricted growth.

\subsubsection{Soft agar assay}

Soft agar assay was performed in 6 well plates coated with $1 \mathrm{ml} 1: 1$ mix of $1 \%$ noble agar in Aqua dest. and cultivation medium. Subsequently, a defined number of PDAC cells in cultivation medium was mixed with 1\% noble agar in Aqua dest. 3:1 and incubated for 30 min under the bench at RT to ensure solidification prior to adding $2 \mathrm{ml}$ cultivation medium on top. TGF $\beta$ was added at this step, utilizing triplicates per condition. The soft agar assay was incubated for 7 days at $37^{\circ} \mathrm{C}$ and $5 \% \mathrm{CO}_{2}$ until final colony counting. Colonies located in 5 different parts per well were counted by focusing through the solid soft agar.

\subsubsection{Sphere assay}

For sphere assays, the cell suspension was centrifuged to remove the FBS-containing medium. Subsequently, cells were resuspended in PBS prior to cell counting. A defined number of cells was plated in low attachment plates (Thermo Fisher Scientific, Corning Costar cell culture plates, 174932) using sphere medium (DMEM/F12 by Gibco, 11320-74) supplemented with both epidermal growth factor (EGF) and fibroblast growth factor (FGF). TGF $\beta$ was directly administered to the medium. Both TGF $\beta$ and FGF treatment was repeated every second day until day 7. Final evaluation was performed by taking 20 random pictures at $200 \times$ magnification per well. Sphere counting and diameter measurement were performed afterward by using FIJI software version 1.52j (Schindelin et al., 2012). 


\subsection{Molecular methods}

\subsubsection{Protein isolation}

For the preparation of whole cell lysate (WCL), the medium was discarded and cells were washed once with ice-cold PBS. Subsequently, WCL buffer supplemented with $1 \mathrm{x}$ cOmplete $^{\text {TM }}$ protease inhibitor cocktail (Roche, 11697498001 ; Stock: 25 x) was added in each well [see WCL composition in table 6]. Cells were scraped, lysates collected in an Eppendorf tube and held on ice for $1 \mathrm{~h}$ with repeated vortexing steps. Cell lysates were centrifugated at maximum speed. The supernatant was transferred into a fresh Eppendorf tube and stored at $-20^{\circ} \mathrm{C}$ until used.

For nuclear and cytoplasmic lysates, cells were either cultivated in $10 \mathrm{~cm}$ or $15 \mathrm{~cm}$ dishes. Upon finalization of the experiment, the medium was discarded and cells were washed once with ice-cold PBS. $1 \mathrm{ml}$ of PBS was added, cells were scraped and lysates transferred to Eppendorf tubes. Subsequent to centrifugation at $0.7 \times \mathrm{g}, 5 \mathrm{~min}$, RT, the supernatant was aspirated and the cell pellet thoroughly resuspended in a corresponding volume of Buffer A supplemented with $1 \times$ cOmplete $^{\mathrm{TM}}$ (Roche, Stock: 25 x) by pipette [see composition of Buffer in table 6]. After 20 min incubation on ice, lysates were centrifugated at $2 \times \mathrm{g}, 15 \mathrm{~min}, 4^{\circ} \mathrm{C}$. The resulting supernatant contains the cytoplasmic fraction and can be transferred to a new Eppendorf tube for storage at $-20^{\circ} \mathrm{C}$. The remaining pellet was solved in Buffer $\mathrm{C}$ [table 6] supplemented with $1 \mathrm{x}$ cOmplete $^{\mathrm{TM}}$ protease inhibitor cocktail (Roche, 11697498001 ; Stock: 25x). Lysates were incubated for $1 \mathrm{~h}$ on ice with repetitive vortexing steps. Lysates were centrifugated at maximum speed, $20 \mathrm{~min}, 4^{\circ} \mathrm{C}$. The supernatant was transferred into a fresh tube and lysates were stored at $-20^{\circ} \mathrm{C}$ until used.

Protein concentrations are measured by comparison to bovine serum albumin (BSA) standard in 1:5 Bradford reagent in Aqua dest. (Bio-Rad, Protein Assay Dye Reagent concentrate, 5000006 ). Note that BSA standard is solved in the respective protein 
buffer used for the corresponding experiment, namely WCL, A, or C buffer (see buffer compositions in table 6).

\subsubsection{Co-immunoprecipitation}

Nuclear lysates were conducted to co-immunoprecipitation studies to investigate nuclear complex formation of transcription factors. PBS supplemented with $1 \times$ cOmplete $^{\mathrm{TM}}$ protease inhibitor cocktail (Roche, 11697498001 ; Stock: 25x) was used to add up $150 \mu \mathrm{g}$ to $200 \mu \mathrm{g}$ protein lysates to $1 \mathrm{ml}$. In addition, input samples were generated from nuclear lysates and stored at $-20^{\circ} \mathrm{C}$. At the same time, slurry sepharose beads (Millipore, 16-125, 16-266) were selected according to table 4, washed three times with PBS and centrifugated at $0.6 \times \mathrm{g}, 2 \mathrm{~min}, 4^{\circ} \mathrm{C}$. At the final washing step, the supernatant was aspirated and beads were resuspended in half of the original volume of beads to create a slurry solution again. For the preclear, $20 \mu \mathrm{l}$ of slurry beads were added to each tube and lysates were incubated at the overhead rotator $(10 \mathrm{rpm}), 4^{\circ} \mathrm{C}$ for $20 \mathrm{~min}$ to reduce unspecific protein binding. Afterward, tubes were centrifugated at $0.6 \times \mathrm{g}$, $5 \mathrm{~min}, 4^{\circ} \mathrm{C}$ and the supernatant carefully transferred to a fresh Eppendorf tube. Antibodies and immunoglobulin $\mathrm{G}$ (IgG) controls were added o/n on the overhead rotator at $10 \mathrm{rpm}, 4^{\circ} \mathrm{C}$ [see table 4$]$.

On the next day, tubes were supplemented with $50 \mu \mathrm{l}$ PBS-washed slurry sepharose beads and incubated at the overhead rotator for $2 \mathrm{~h}$. Subsequently, beads were spun down $\left(0.6 \times \mathrm{g}, 4^{\circ} \mathrm{C}, 5 \mathrm{~min}\right)$ and the supernatant was aspirated. Beads were washed five times with $500 \mu \mathrm{l}$ WCL buffer and three times with $500 \mu \mathrm{l}$ PBS. Finally, the supernatant was carefully completely aspirated and beads were incubated with $75 \mu \mathrm{l}$ $2 \times$ Laemmli buffer (stock: $5 \times$, diluted in aqua bidest) for 5 min at $95^{\circ} \mathrm{C}$ before allow cooling down on ice. This step was repeated once, then samples were spun down and the supernatant was transferred without any beads to a fresh tube. Samples were either stored at $-20^{\circ} \mathrm{C}$ or directly used for western blot in combination with the input samples prepared at the start. 
Table 4 Antibodies for co-immunoprecipitation experiments. Indicated antibodies were used for co-IP studies in nuclear lysates of human and murine PDAC cells. Volume of normal rabbit/mouse IgG was adjusted (adj.) to respective volume of antibody.

\begin{tabular}{llcccc}
\hline Target & Company & Order number & Species & Volume $[\mu l]$ & Beads \\
\hline Flag-tag & Sigma & F3165-.2MG & mouse & 3 & $\mathrm{G}$ \\
HA-tag & abcam & ab75640 & rabbit & 5 & $\mathrm{~A}$ \\
HA-tag & CST & 3724 & rabbit & 5 & $\mathrm{~A}$ \\
NFATc1 & Santa Cruz & sc-13033 & rabbit & 15 & $\mathrm{~A}$ \\
IgG & Santa Cruz & sc- 2025 & mouse & adj. & $\mathrm{G}$ \\
IgG & Millipore & $12-370$ & rabbit & adj. & $\mathrm{A}$ \\
Smad3 & abcam & ab28379 & rabbit & 10 & $\mathrm{~A}$ \\
\hline
\end{tabular}

\subsubsection{Western Blot}

Protein samples solved in WCL buffer were adjusted to $1 \mu \mathrm{g} / \mathrm{ml}$ and $5 \times$ Laemmli buffer supplemented with $5 \% \beta$-mercaptoethanol was added. Prepared samples were placed at $95^{\circ} \mathrm{C}$ for $5 \mathrm{~min}$ with subsequent cooling on ice.

Protein separation according to size was realized using sodium dodecyl sulfate polyacrylamide gel electrophoresis (SDS-PAGE) approaches. For gel preparation, SDS-PAGE, and blotting, Bio-Rad equipment was used. Gel preparation was conducted according to the expected protein sizes, choosing $15 \%$ gels for small sized proteins $(<30$ $\mathrm{kDa}$ ) and $10 \%$ gels for big proteins $(30 \mathrm{kDa}<\mathrm{x}<180 \mathrm{kDa})$, respectively [table 5]. $20 \mu \mathrm{g}$ of the respective sample and $4 \mu \mathrm{l}$ Page Ruler Prestained Protein Ladder (Thermo Fisher Scientific, 26617) were loaded before gel run in $1 \times$ Electrophoresis Buffer [see composition in table 6].

Gels were released from the chamber and conducted to semi-dry turbo blotting using the Trans-Blot ${ }^{\circledR}$ Turbo ${ }^{\text {TM }}$ Transfer System (Bio-Rad; 1704150 ) according to the manufacturer's instructions (Bio-Rad; Trans-Blot ${ }^{\circledR}$ ) Turbo ${ }^{\text {TM }}$ RTA Midi Nitrocellulose Transfer Kit, 1704271$)$. Blotting time was adjusted according to desired protein sizes (fixed: $1.0 \mathrm{~A}, 25 \mathrm{~V}$ ). $15 \%$ gels were blotted for $10 \mathrm{~min}$ and $10 \%$ gels were blotted for $22 \mathrm{~min}$. The nitrocellulose membrane was stained with Ponceau S solution (Sigma- 
Table 5 Composition of gel solutions. All gel buffers were stored at $4{ }^{\circ} \mathrm{C}$. Abbreviations: sodium dodecyl sulfate (SDS), tris(hydroxymethyl)aminomethan (Tris), and volume per volume (v/v).

\begin{tabular}{llr}
\hline Solution & Composition & Amount \\
\hline Stacking Gel Buffer (stock) & Tris-base & $0.5 \mathrm{M}$ \\
pH 6.8 & SDS & $0.4 \%$ (v/v) \\
Stacking Gel Buffer & Stacking Gel Buffer (Stock) & $25 \mathrm{ml}$ \\
& Acrylamide & $16 \mathrm{ml}$ \\
& Aqua dest. & $59 \mathrm{ml}$ \\
\hline Separating Gel Buffer (stock) & Tris-base & $1.5 \mathrm{M}$ \\
pH 8.8 & SDS & $0.4 \%(\mathrm{v} / \mathrm{v})$ \\
Separating Gel Buffer (10\%) & Separating Gel Buffer (Stock) & $20 \mathrm{ml}$ \\
& Acrylamide & $26.6 \mathrm{ml}$ \\
& Glycerol & $4 \mathrm{ml}$ \\
& Aqua dest. & $29.3 \mathrm{ml}$ \\
Separating Gel Buffer (15\%) & Separating Gel Buffer (Stock) & $20 \mathrm{ml}$ \\
& Acrylamide & $40 \mathrm{ml}$ \\
& Glycerol & $4 \mathrm{ml}$ \\
& Aqua dest. & $16 \mathrm{ml}$ \\
\hline
\end{tabular}

Aldrich Corporation; P7170) to fix proteins on the membrane and their visualization. Subsequent to $1 \mathrm{~h}$ blocking in Tris-buffered saline (TBS) supplemented with $1 \%$ Tween and $5 \%$ milk powder at RT, membranes were incubated in primary antibodies using dilutions described in table $7 \mathrm{o} / \mathrm{n}$ at $4{ }^{\circ} \mathrm{C}$.

On the following day, antibody dilutions were removed from the membranes and replaced by TBS supplemented with $1 \%$ Tween in three washing steps at RT. Afterward, horseradish peroxidase-linked (HRP) secondary antibodies (Cell Signaling Technology (CST); $\alpha$-rabbit IgG HRP-linked antibody \#7074, $\alpha$-mouse IgG HRPlinked antibody \#7076) were chosen according to the primary antibody's species and diluted 1:5000 in TBS supplemented with 1\% Tween and 5\% milk powder at RT for $1.5 \mathrm{~h}$. Membranes were washed three times with TBS supplemented with 1\% Tween before protein detection by enhanced chemiluminescence (ECL) method with ECL Plus (PerkinElmer; NEL105 001EA) or Western Lightning Ultra (PerkinElmer; 
Table 6 Buffers for co-IP and SDS-PAGE. Ingredients were solved in Aqua dest.. Buffer A, C, and WCL need to be supplemented with proteinase inhibitors prior to usage. Abbreviations: ethylenediaminetetraacetic acid (EDTA), ethylene glycolbis(ß-aminoethyl ether)-N,N,N',N'-tetraacetic acid (EGTA), 4-(2hydroxyethyl)-1-piperazineethanesulfonic acid (HEPES), and weight per volume $(\mathrm{w} / \mathrm{v})$.

\begin{tabular}{lll}
\hline Buffer & Composition & Concentration \\
\hline Buffer A & HEPES pH 7.9 & $10 \mathrm{mM}$ \\
& KCl & $10 \mathrm{mM}$ \\
EDTA & $100 \mu \mathrm{M}$ \\
EGTA & $100 \mu \mathrm{M}$ \\
Buffer C & HEPES pH 7.9 & $20 \mathrm{mM}$ \\
& NaCl & $400 \mathrm{mM}$ \\
Electrophoresis Buffer & EGTA & $1 \mathrm{mM}$ \\
& Tris-base & $1 \mathrm{mM}$ \\
TBS (pH 7.6) & Glycine & $1.92 \mathrm{M}$ \\
SDS & $1 \%(\mathrm{w} / \mathrm{v})$ \\
& Tris-base & $0.2 \mathrm{M}$ \\
& NaCl & $1.37 \mathrm{M}$ \\
& HEPES pH 7.5 & $50 \mathrm{mM}$ \\
& NaCl & $150 \mathrm{mM}$ \\
& EGTA & $1 \mathrm{mM}$ \\
& Glycerol & $10 \%(\mathrm{v} / \mathrm{v})$ \\
& Triton X-100 & $1 \%(\mathrm{v} / \mathrm{v})$ \\
& NaF & $100 \mathrm{mM}$ \\
& Na $\mathrm{P}_{2} \mathrm{O}_{7} \times 10 \mathrm{H}_{2} \mathrm{O}$ & $10 \mathrm{mM}$ \\
\hline
\end{tabular}

NEL112 001EA) at Intas ECL Chemocam Imager (Intas Science Imaging Instruments). Protein bands were processed using chemostar software (Intas Science Imaging Instruments) and GIMP software (GIMP (1997), version 2.8.22), while analysis was performed by using FIJI software (Schindelin et al., 2012).

For co-IP blots with targets at 35 to $55 \mathrm{kDa}$ size, membranes were incubated with $\alpha$-rabbit light chain specific mouse antibody (CST, \#3677) in 1:2000 dilution in TBS supplemented with $1 \%$ Tween and $5 \%$ milk powder at RT for $1 \mathrm{~h}$ prior to incubation 
Table 7 Antibodies for western blot. Antibodies were diluted in 5\% milk powder in TBS supplemented with $1 \%$ Tween. Membranes were incubated in respective antibody solutions o/n at $4{ }^{\circ} \mathrm{C}$.

\begin{tabular}{lcccl}
\hline Target & Company & Number & Species & Dilution \\
\hline ß-Actin-HRP & Sigma & A3854 & & $1: 40000$ \\
cJun & CST & 9165 & rabbit & $1: 1000$ \\
Erk1/2 & CST & 9102 & rabbit & $1: 1000$ \\
pErk1/2 & CST & 9101 & rabbit & $1: 1000$ \\
Flag-HRP & Sigma & A8592 & & $1: 1000$ \\
HA-Tag & CST & 2367 & mouse & $1: 1000$ \\
HA-Tag & CST & 3724 & rabbit & $1: 1000$ \\
Hdac1 & CST & 2062 & rabbit & $1: 1000$ \\
Hdac1 & abcam & ab33278 & rabbit & $1: 1000$ \\
NFATc1 & Santa Cruz & sc- 7294 & mouse & $1: 500$ \\
NFATc1 & Santa Cruz & sc- 13033 & rabbit & $1: 500$ \\
Parp & CST & 9542 & rabbit & $1: 1000$ \\
Smad2 & CST & 5339 & rabbit & $1: 1000$ \\
Smad2/3 & BDiosciences & 610842 & mouse & $1: 500$ \\
Smad2/3 & CST & 3102 & rabbit & $1: 1000$ \\
pSmad2/3 & CST & 8828 & rabbit & $1: 1000$ \\
Smad3 & CST & 9523 & rabbit & $1: 1000$ \\
pSmad3 & CST & 9520 & rabbit & $1: 1000$ \\
Smad4 & Santa Cruz & sc-7966 & mouse & $1: 500$ \\
Snai1 & CST & 3895 & mouse & $1: 1000$ \\
\hline
\end{tabular}

with $\alpha$-mouse HRP-coupled secondary antibody (CST, \#7076) in 1:5000 dilution in TBS supplemented with $1 \%$ Tween and $5 \%$ milk powder at RT for $1.5 \mathrm{~h}$ to avoid heavy chain detection. Note that in this approach it is crucial to perform co-IP using a rabbit-derived antibody and to choose a rabbit-derived primary antibody on the membrane.

\subsubsection{RNA isolation}

Cells were seeded in 6 wells and treated according to the experimental design. Medium was aspirated and $500 \mathrm{\mu l}$ TRIzol (ambion, 15596018 ) was added per well. After $5 \mathrm{~min}$ incubation at RT, cells were scraped and transferred into an Eppendorf tube for storage 
at $-80^{\circ} \mathrm{C}$. For RNA isolation, TRIzol lysates were thawed on ice and $200 \mu \mathrm{l}$ chloroform was added. Subsequent to rigorous vortexing, the tubes were incubated for $10 \mathrm{~min}$ at RT. Phase separation was achieved by centrifugation at maximum speed for 15 min at $4{ }^{\circ} \mathrm{C}$. The transparent upper phase was carefully transferred to a fresh tube, $500 \mu \mathrm{l}$ isopropyl alcohol was added and the tube was inverted until proper phase mixing. Tubes were placed in the centrifuge at maximum speed for $30 \mathrm{~min}$ at $4{ }^{\circ} \mathrm{C}$. The supernatant was carefully aspirated and replaced by $500 \mu \mathrm{l} 75 \%$ undenatured ethanol. Upon rigorous vortexing, tubes were centrifuged at maximum speed for $5 \mathrm{~min}$ at $4{ }^{\circ} \mathrm{C}$. The supernatant was carefully discarded and tubes were incubated with open lids at RT until remaining ethanol was evaporated. RNA pellet was resuspended in $30 \mu \mathrm{l}$ to $50 \mu \mathrm{l}$ RNase-free aqua bidest. RNA was stored at $-80^{\circ} \mathrm{C}$ until used.

\subsection{5 qRT-PCR}

To evaluate and compare gene expression levels, quantitative real time polymerase chain reaction (qRT-PCR) was performed. Therefore, RNA was transcribed into complementary deoxyribonucleic acid (cDNA) using iScript ${ }^{\mathrm{TM}} \mathrm{cDNA}$ Synthesis Kit (Bio-Rad; 1708890 ) according to the manufacturer's suggestions. For the qRT-PCR approach, cDNA was supplemented with iTaq ${ }^{\mathrm{TM}}$ Universal SYBR $^{\circledR}$ Green Supermix (Bio-Rad; 172-5121) and specific human or murine messenger ribonucleic acid (mRNA) primers [see reaction mix in table 8 and primer sequences in table 9]. The experiments were conducted in three technical replicates using a 96 well plate (Applied Biosystems by Thermo Fisher Scientific, MicroAmp Fast 96-Well Reaction Plate, 4346 907), sealed with a film (Applied Biosystems by Thermo Fisher Scientific, MicroAmp Optical Adhesive Film, 4311971) and placed in a StepOne Real-Time PCR system (Applied Biosystems) running the standard protocol. Data were analyzed using normalization to the house keeping gene Rplp0 via the $\Delta \Delta \mathrm{CT}$ approach. 
Table 8 Reaction mix for qRT-PCR experiments. Reaction mix was pipetted into a 96 well plate (Applied Biosystems), sealed and placed in a StepOne system (Applied Biosystems).

\begin{tabular}{lc}
\hline Ingredients & $\mathbf{1 \times}$ \\
\hline Sybr Green & $5 \mu \mathrm{l}$ \\
Primer forward & $0.05 \mu \mathrm{l}$ \\
Primer reverse & $0.05 \mu \mathrm{l}$ \\
Aqua bidest & $3.9 \mu \mathrm{l}$ \\
cDNA & $1 \mu \mathrm{l}$ \\
\hline
\end{tabular}

Table 9 Primer sequences for qRT-PCR experiments. Primers were diluted to $10 \mu \mathrm{M}$ in Aqua dest. prior to preparation of reaction mix [see table 8].

\begin{tabular}{lcl}
\hline Target & Direction & Sequence \\
\hline Cda & forward & 5'-TCA GCC TAC TGC CCC TAC AG-3' \\
reverse & 5'-AGC AGG CGT TTT CTA TGT TGC-3' \\
Cdc20 & forward & 5'-CTG TCT GAG TGC TGT GGA TG-3' \\
& reverse & 5'-AAA GCC GTG ACC TGA GAT C-3' \\
Cks1b & forward & 5'-CAC TGC CCA AGA AGC CAA AG-3' \\
& reverse & 5'-ACA TGG TCA CGC GAT CAG AA-3' \\
Dctd & forward & 5'-CAA ACC TCA CTG GGA ACC GTA-3' \\
& reverse & 5'-GGG AGC CAG AGC TTA TCC C-3' \\
Fgfbp1 & forward & 5'-ACT CAC AGA AAG GCA CTT GGA-3' \\
& reverse & 5'-CTG AGA ACG CCT GAG TAG CC-3' \\
Mcm4 & forward & 5'-GTG AGG AAA GCA GGT CGT CA-3' \\
Nfatc1 & reverse & 5'-ACA AGG CAT TCT GTG CAG GT-3' \\
& forward & 5'-GCC TTT TGC GAG CAG TAT CT-3' \\
Rrm1 & reverse & 5'-GCT GCC TTC CGT CTC ATA GT-3' \\
& forward & 5'-ATC TTA CTG AGC GGG GCT TG-3' \\
Rrm2 & reverse & 5'-ATG AAA GCA CCT CTC TCG GC-3' \\
& forward & 5'-TTT GCC GCC GTA GAA GGA AT-3' \\
Rplp0 & reverse & 5'-ATC AGC CCC CGT TTC TTG AG-3' \\
Smad4 & forward & 5'-TGG GCA AGA ACA CCA TGA TG-3' \\
Smad7 & reverse & 5'-AGT TTC TCC AGA GCT GGG TTG T-3' \\
& forward & 5'-GTG ATC TAT GCC CGT CTG TG-3' \\
& reverse & 5'-ATT ACT CTG CAG TGT TAA TC-3' \\
& forward & 5'-TGC TCC CAT CCT GTG TGT TAA G-3' \\
& reverse & 5'-TCA GCC TAG GAT GGT ACC TTG G-3' \\
\hline
\end{tabular}




\subsection{RNA sequencing and bioinformatic analyses}

Prior to library preparation for RNA sequencing (RNAseq), RNA integrity and purity was evaluated by separation and visualization on an $1 \%$ agarose gel supplemented with Midori Green Advance (Biozym, 617 004).

Library preparation was performed using Illumina kits (RS-122-2001; RS-122-2002) according to the manufacturer's instructions. Qubit measurement (Thermo Scientific, Q32 854) controlled cDNA concentrations and fragment sizes were measured by Agilent 2100 bioanalyzer high sensitivity DNA analysis kit (Agilent, 5067-4626) utilizing Agilent 2100 Expert software. Subsequent to preparation of $5 \mathrm{nM}$ mastermix, the sample was given for sequencing to Transcriptome and Genome Analysis Laboratory of the University Medical Center Göttingen, Germany.

RNAseq data were uploaded to the public server at usegalaxy.org (Afgan et al., 2018). TopHat tool (Kim et al., 2013) (version 2.1.1) with very sensitive bowtie2 settings and subsequent sorting by SortSam (version 2.18.2.1) was used to map Fastq files to the murine transcriptome (mm9). After read counting via HTSeq (Anders et al., 2015) (union mode; version 0.9.1), DESeq2 (Anders \& Huber, 2010; Love et al., 2014) was employed to measure principal component analysis (PCA) and sample-to-sample distances for assessing similarities between replicates. Cuffdiff (version 2.2.1.5) and Cuffnorm tools (geometric; version 2.2.1.2) conveyed differential regulation analysis and normalized fragment counts, respectively (Trapnell et al., 2012; Ghosh \& Chan, 2016). For further analysis, fragments per kilobase million (FPKM) threshold $>5$ was established to reduce background. Heatmaps are based on z scores of each replicate. These data were processed using hierarchical clustering by Cluster 3.0 (Euclidean distance; version 1.54) in combination with Java TreeView (version 1.1.6r4) (Saldanha, 2004).

Potential NFATc1/Smad3/cJun targets were conducted to gene ontology (GO) analysis (Ashburner et al., 2000; The Gene Ontology Consortium, 2017) to determine their 
functional relevance. The overrepresentation test of protein analysis through evolutionary relationships (PANTHER; version 14.0) was employed with Fisher's exact test using either Bonferroni correction for multiple testing ( $p$ value) or the false discovery rate (FDR) to assess the functional relevance by classification with the GO biological process complete algorithms (Thomas et al., 2003; Mi et al., 2013).

Datasets for genes associated in unfavorable or favorable prognosis in PDAC were extracted from the human protein atlas (Cancer Genome Atlas Research Network et al., 2013; Uhlen et al., 2017) and processed by gene set enrichment analysis (GSEA). GSEA (Mootha et al., 2003; Subramanian et al., 2005) was conducted with Signal2Noise metric and FDR $<0.25$ was considered significant. Normalized enrichment score (NES) served as the primary statistic.

To compare murine Smad4-dependent survival data to human PDAC patients, The Cancer Genome Atlas (TCGA) data (Cancer Genome Atlas Research Network, 2017) were analyzed with OncoLnc tool (Anaya, 2016) by setting percentiles at 25 (patient groups $\mathrm{n}=43$, respectively).

\subsection{Genetically engineered mouse models}

Functional assays and pathway analysis performed in cell culture were expanded by in vivo studies employing GEMMs of Smad4 deficiency.

\subsubsection{GEMM breeding}

Investigating implications of Smad4 deficiency in vivo was executed by both employing mice harboring flox/+ (heterozygous) (fl/+) or flox/flox (homozygous) (fl/fl) depletion of Smad4. Smad4 fl/fl mice as described by Yang et al. (2002) were ordered by Charles River Laboratories, Germany. Interbreeding with mice bearing p48-Cre recombinase (Hingorani et al., 2003) resulted in conditional deletion of $\operatorname{Smad} 4$ exon 8 in the pan- 
creas, leading to protein misfolding and premature degradation. In our animal breeding system, both homozygous and heterozygous Smad4 depletion was introduced to mice bearing conditional $\operatorname{Kras}^{G 12 D}$ activation controlled by p48-Cre (Yang et al., 2002; Hingorani et al., 2003). This resulted in $\operatorname{Smad}^{\mathrm{H} /+} ; \mathrm{Kras}^{\mathrm{G} 12 \mathrm{D}} ; \mathrm{p} 48-\mathrm{Cre}(\mathrm{SKC} \mathrm{Hl}+\mathrm{p} 48)$ and $S m a d 4^{f / f l} ; \operatorname{Kras}^{G 12 D}$; p48-Cre (SKC fl/fl p48) mice that were analyzed in survival studies or sacrificed at defined time points (4 weeks, 8 weeks, 12 weeks, and 6 months, respectively). Kras WT littermates were employed in the same studies as controls. The following end-point criteria were employed for survival studies: development of ascites, body weight loss (>20\%), lethargy or lack of social behavior. Importantly, Kras ${ }^{G 12 D}$; p48-Cre (KC p48) control animals were bred and sacrificed as part of the medical doctorate thesis of B. Steuber, Department of Gastroenterology and Gastrointestinal Oncology, University Medical Center Göttingen.

Mouse genotyping was performed by DNA isolation from tail or ear biopsies. Respective biopsies were incubated in Alkaline Lysis Buffer (25 mM NaOH, 0.2 mM EDTA) at $95^{\circ} \mathrm{C}$ for $30 \mathrm{~min}$ before adding the same volume of Neutralization Buffer (40 mM Tris$\mathrm{HCl}$ ). Samples were stored at $-20^{\circ} \mathrm{C}$.

Table 10 Primer sequences for genotyping. Primers were diluted to $10 \mu \mathrm{M}$ prior to addition to $\mathrm{PCR}$ reaction mix.

\begin{tabular}{lcl}
\hline Target & Direction & Sequence \\
\hline Smad4 & forward & 5'-TAA GAG CCA CAG GGT CAA GC-3' \\
& reverse & 5'-TTC CAG GAA AAA CAG GGC TA-3' \\
Kras & forward & 5'-AGC TAG CCA CCA TGG CTT GAG TAA GTC TGC G-3' \\
& reverse & 5'-CCT TTA CAA GCG CAC GCA GAC TGT AGA-3' \\
p48-Cre & forward & 5'-TGC TGT TTC ACT GGT TAT GCG G-3' \\
& reverse & 5'-TTG CCC CTG TTT CAC TAT CCA G-3' \\
\hline
\end{tabular}

Mouse strains were genotyped by PCR with subsequent band separation by PerfectBlue Horizontal Maxi Gel Systems (Peqlab, 700-540) and visualization by agarose gel at ultraviolet transilluminator (Bio-Rad, ChemiDoc XRS+). PCR mix (Nippon Genetics, KK5621, KAPA2G Fast Hot Start Genotyping Mix with Dye) was supplemented with DNA derived from mice tail biopsies and genotyping primers depicted in table 10 
Table 11 Reaction mix for genotyping PCR. PCR was performed in T100 Thermal Cycler (Bio-Rad) and the product was subsequently separated by agarose gel.

\begin{tabular}{lc}
\hline Ingredients & $\mathbf{1 \times}$ \\
\hline KAPA2G & $6.25 \mu \mathrm{l}$ \\
Primer forward & $0.5 \mu \mathrm{l}$ \\
Primer reverse & $0.5 \mu \mathrm{l}$ \\
Aqua bidest & $4.25 \mu \mathrm{l}$ \\
Genomic DNA & $1 \mu \mathrm{l}$ \\
\hline
\end{tabular}

Table 12 PCR protocols. The following PCR programs were realized on T100 Thermal Cycler (Bio-Rad) and the product was subsequently separated by agarose gel. (a) shows the protocol for the validation of potential CRISPR/Cas9 k.o. and WT clones, while $(b+c)$ refer to genotyping PCRs validating the respective GEMMs.

(a) CRISPR/Cas9 validation

\begin{tabular}{cc}
\hline Temperature & Time \\
\hline $95^{\circ} \mathrm{C}$ & $3 \mathrm{~min}$ \\
$95^{\circ} \mathrm{C}$ & $30 \mathrm{sec}$ \\
$35 \times \quad 63^{\circ} \mathrm{C}$ & $30 \mathrm{sec}$ \\
$72^{\circ} \mathrm{C}$ & $30 \mathrm{sec}$ \\
$72^{\circ} \mathrm{C}$ & $1 \mathrm{~min}$ \\
\hline
\end{tabular}

(b) Kras and p48-Cre

\begin{tabular}{cc}
\hline Temperature & Time \\
\hline $95{ }^{\circ} \mathrm{C}$ & $2 \mathrm{~min}$ \\
$95^{\circ} \mathrm{C}$ & $30 \mathrm{sec}$ \\
$35 \times \quad 60^{\circ} \mathrm{C}$ & $30 \mathrm{sec}$ \\
$72^{\circ} \mathrm{C}$ & $60 \mathrm{sec}$ \\
$72^{\circ} \mathrm{C}$ & $5 \mathrm{~min}$ \\
\hline
\end{tabular}

(c) Smad4

\begin{tabular}{|c|c|c|}
\hline \multicolumn{2}{|c|}{ Temperature } & Time \\
\hline \multicolumn{2}{|c|}{$94^{\circ} \mathrm{C}$} & $2 \mathrm{~min}$ \\
\hline $10 x$ & $94^{\circ} \mathrm{C}$ & $20 \mathrm{sec}$ \\
\hline$-1.5^{\circ} \mathrm{C} /$ cycle & $65^{\circ} \mathrm{C}$ & $15 \mathrm{sec}$ \\
\hline \multicolumn{2}{|c|}{$68^{\circ} \mathrm{C}$} & $10 \mathrm{sec}$ \\
\hline \multirow{3}{*}{$28 x$} & $94^{\circ} \mathrm{C}$ & $15 \mathrm{sec}$ \\
\hline & $50^{\circ} \mathrm{C}$ & $15 \mathrm{sec}$ \\
\hline & $72^{\circ} \mathrm{C}$ & $10 \mathrm{sec}$ \\
\hline \multicolumn{2}{|c|}{$72{ }^{\circ} \mathrm{C}$} & $2 \mathrm{~min}$ \\
\hline
\end{tabular}


[reaction mix in table 11]. Subsequent to PCR in T100 Thermal Cycler (Bio-Rad, 186-1096) [see PCR protocol in table 12], samples were loaded on respective agarose gels supplemented with Midori Green Advance. Note that Smad4 primers produce a double band on a $2 \%$ agarose gel in heterozygous k.o. as Smad4 WT PCR product's length is 436 base pair (bp) and mutated Smad4 500 bp.

All procedures were carried out using protocols approved by the Institutional Animal Care and Use Committee at the University Medical Center Göttingen and respective mice are listed under the animal approval number 33.9-42502-04-14/1634.

\subsubsection{Tissue processing}

Mice were sacrificed using $\mathrm{CO}_{2}$ prior to cervical dislocation and heart disruption. Subsequent to opening the abdomen, pancreatic tissue was isolated and divided into 4 parts from head to tail. While part 1 and 3 were immediately snap frozen in liquid nitrogen for later RNA or protein isolation, the remaining tissue was incubated in $4 \%$ paraformaldehyde in PBS for $24 \mathrm{~h}$ at RT for tissue fixation. Additionally, parts of liver and spleen were added for fixation. The next day, tissue in paraformaldehyde solution underwent a dehydration series performed by Leica Tissue Processor (TP1020). The last steps included incubation in paraffin and tissue block preparation using Leica embedding system (EG1150H). Tissue blocks were allowed to solidify at HistoCore Arcadia C cooling plate (Leica, 14600005015 ) prior to storage at RT in the dark.

Sectioning by microtome (Leica, RM2265) was performed by applying $4 \mu \mathrm{m}$ sections on slides with subsequent incubation at $37^{\circ} \mathrm{C} \mathrm{o} / \mathrm{n}$ prior to storage at $\mathrm{RT}$ in the dark.

\subsubsection{HE and IHC}

For hematoxylin and eosin (HE) and IHC staining, tissue sections passed through a rehydration series starting with Roticlear (Carl Roth, A538) transferring to Ethanol 
dilutions, ending in tap water. For HE stainings, slides were then dyed in hematoxylin solution according to Mayer (Sigma, 51275 ) with subsequent incubation under running tap water. Next, sections were counterstained with eosin y solution (Sigma-Aldrich, HT110 232) prior to dehydration series ending in Roticlear. Cover slips were mounted using mounting medium (Carl Roth, HP68.2).

Table 13 Composition of antigen retrieval buffers. Buffers were filled up to 11 with Aqua bidest.

\begin{tabular}{llr}
\hline Solution & Components & Amount \\
\hline Citrate Buffer & Citric acid monohydrate & $2.1 \mathrm{~g}$ \\
pH 6 & & \\
TE Buffer $(10 \mathrm{x})$ & Tris-Base & $12.1 \mathrm{~g}$ \\
pH 9 & EDTA & $3.7 \mathrm{~g}$ \\
\hline
\end{tabular}

Table 14 Composition of washing buffer. Buffer was filled up to 51 with Aqua bidest.

\begin{tabular}{llr}
\hline Solution & Components & Amount \\
\hline PB washing buffer & $\mathrm{NaH}_{2} \mathrm{PO}_{4} \times \mathrm{H}_{2} \mathrm{O}$ & $12.05 \mathrm{~g}$ \\
pH 7.4 & $\mathrm{Na}_{2} \mathrm{HPO}_{4} \times 2 \mathrm{H}_{2} \mathrm{O}$ & $56.7 \mathrm{~g}$ \\
PBS washing buffer & $\mathrm{KCl}$ & $1 \mathrm{~g}$ \\
pH 7.2 to 7.4 & $\mathrm{KH}_{2} \mathrm{PO}_{4}$ & $1 \mathrm{~g}$ \\
& $\mathrm{Na}_{2} \mathrm{HPO}_{4} \times 2 \mathrm{H}_{2} \mathrm{O}$ & $3.8 \mathrm{~g}$ \\
& $\mathrm{NaCl}$ & $40 \mathrm{~g}$ \\
\hline
\end{tabular}

IHC stainings required heat-induced epitope retrieval using either citrate or Tris EDTA (TE) buffer indicated in table 13. Subsequently, slides were allowed to cool down in their respective antigen retrieval buffers in an ice box. Signal of endogenous peroxidase was reduced by incubation in $3 \%$ hydrogen peroxide solution. Slides were aligned into Sequenza $^{\mathrm{TM}}$ Slide Rack and washed with PBS supplemented with $1 \%$ Tween-20 or Triton X-100 depending on the primary antibody (table 14). Unspecific binding was reduced by incubation with $10 \%$ BSA in the respective washing buffer prior to adding the primary antibody diluted in the same solution. For PDAC staining, we employed NFATc1 (abcam, ab2796; citrate buffer; 1:100 in PBS-Triton X-100), Smad3 
(abcam, ab28379; TE buffer; 1:500 in PBS-Tween-20) and Smad4 antibody (Santa Cruz, sc-7966; citrate buffer; 1:200 in PBS-Tween-20), respectively. Murine slides were stained for Smad4 using an alternative antibody (CST, 46 535; citrate buffer; 1:200 in PBS-Triton X-100). Primary antibody was incubated o/n at $4{ }^{\circ} \mathrm{C}$. The next day, slides were washed with their respective washing buffer prior to secondary antibody prior to Streptavidin-Biotin complex incubation according to the manufacturer's protocol (Biozol, Vectastain ABC Kit, PK-4001 and PK-4002). Subsequently, 3,3'diaminobenzidine (DAB) staining (Vector, DAB ImmPACT, SK-4105) was precisely timed and counterstained by hematoxylin before dehydration series and mounting.

\subsubsection{IF and PLA}

For immunofluorescence (IF) and proximity ligation assay (PLA) experiments, murine PDAC slides were rehydrated as described in 2.5.3. Heat-activated antigen retrieval was performed using citrate buffer. Tissue was encircled by Dako Pen (Agilent, S200 2302) and washed with phosphate buffer $(\mathrm{PB})+0.4 \%$ Triton $\mathrm{X}-100$ prior to $1 \mathrm{~h}$ blocking with $10 \%$ normal goat serum in PB $+0.4 \%$ Triton X-100 at RT. NFATc1 (abcam, ab2796; 1:100) and Smad3 (CST, 9523; 1:100) primary antibodies were incubated o/n at $4^{\circ} \mathrm{C}$.

For IF stainings, secondary antibodies (abcam, ab175 473 and ab; 1:1000) were incubated for $2 \mathrm{~h}$ at RT prior to 4',6-diamidino-2-phenylindole (DAPI) staining (SigmaAldrich, D9542; 1:2000) and cover slip mounting (Thermo Scientific, 9990402 ).

The second day of PLA experiments was conducted using the Duolink ${ }^{\circledR}$ in situ Kit according to the manufacturer's protocol (Sigma, DUO92 101). Mounting was performed using DAPI-supplemented mounting medium (Vector, Vectashield, H-1200). IF and PLA stainings were allowed to dry for $2 \mathrm{~h}$ at $4{ }^{\circ} \mathrm{C}$ in the dark. 



\section{Results}

In this work we investigated the interplay of the inflammatory transcription factor NFATc1 with active TGF $\beta$ signaling in PDAC. To scrutinize if and under which specific conditions NFATc1 and SMAD3 directly interact, we developed several model systems covering different genetic aspects distinctive for PDAC heterogeneity. In the first part, we scrutinized how loss of SMAD4 drives PDAC development by altering the functional characteristics of the cell. In this context, we elucidated conditions favorable for nuclear NFATc1/SMAD3 complex assembly by employing PLA and co-IP approaches. The second part provided insights into chemotherapeutic resistance and sensitivities as a consequence of SMAD4 deficiency and highlighted the crucial role of AP-1 proteins for nuclear NFATc1/SMAD3 complex formation. Pathways regulated by NFATc1/SMAD3 transcription complexes were examined via genome-wide expression studies.

\subsection{Nuclear NFATc1/Smad3 complexes in Smad4-deficient PDAC}

To understand the implications of Smad4 depletion in the context of oncogenic Kras activation in the pancreas, we first explored murine in vivo models to assess loss of Smad4 as a tumorigenic driver in the context of NFATc1 WT expression. Secondly, we employed CRISPR/Cas9-mediated genome editing to investigate complete loss of Smad4 in epithelial tumor cell lines in vitro. Both approaches aimed to scrutinize the conditions shaping the interplay of NFATc1 and Smad3 transcription factors in PDAC. 


\subsubsection{A murine model of Smad4 deficiency recapitulates features of human PDAC}

First of all, we employed human PDAC data gathered by TCGA (Cancer Genome Atlas Research Network, 2017; Anaya, 2016) to check for SMAD4-dependent patient survival. Comparing SMAD4 low and high expressing PDAC-derived patient samples, we confirmed Smad4 deficiency being associated with dismal prognosis [Figure 4 A], thus suggesting SMAD4 deficiency as a prognostic marker for accelerated PDAC progression and decreased outcome (394d versus $476 \mathrm{~d}$, respectively).

Investigating Smad4-dependent tumorigenesis in PDAC included the generation of a GEMM that either maintained Smad4 WT status (KC p48), harbored a heterozygous Smad4 k.o. ( $S K C \mathrm{fl} /+\mathrm{p} 48$ ) or homozygous Smad4 k.o. (SKC fl/fl p48). Importantly, we controlled how and to which extend homozygous and heterozygous Smad4 depletion could be visualized via IHC [Figure 5]. KC p48 mice demonstrated Smad4 positive nuclei of PanIN lesions, while in SKC fl/fl p48 mice Smad4 positive stromal cells served as staining control. However, partial Smad4 depletion in $\mathrm{SKC} \mathrm{fl} / \mathrm{p} 48$ mice did not result in abrogation of $\operatorname{Smad} 4$ protein expression in the nuclei of PanINs.

The KC p48 mouse first introduced by Hingorani et al. (2003) is an established model for slowly progressing PDAC with a median survival of $493 \mathrm{~d}$ [Figure $4 \mathrm{~B}$ ]. In contrast, upon introduction of conditional Smad4 k.o. animals by Yang et al. (2002), there have been only a few murine Smad4 deficiency studies in the pancreas (Izeradjene et al., 2007; Kojima et al., 2007; Whittle et al., 2015) covering different Cre recombinases and p53 conditions. Our mouse model faithfully recapitulated Smad4-dependent survival as already observed in human PDAC patients, with reduced Smad4 expression being associated with decreased GEMM lifespan ( $S K C$ fl/fl p48: $129 \mathrm{~d}$ ). In contrast to Izeradjene et al. (2007), we already noticed significant reduction of median survival upon heterozygous Smad4 depletion (307.5 d) compared to Smad4 WT-bearing KC p48 animals. 
A
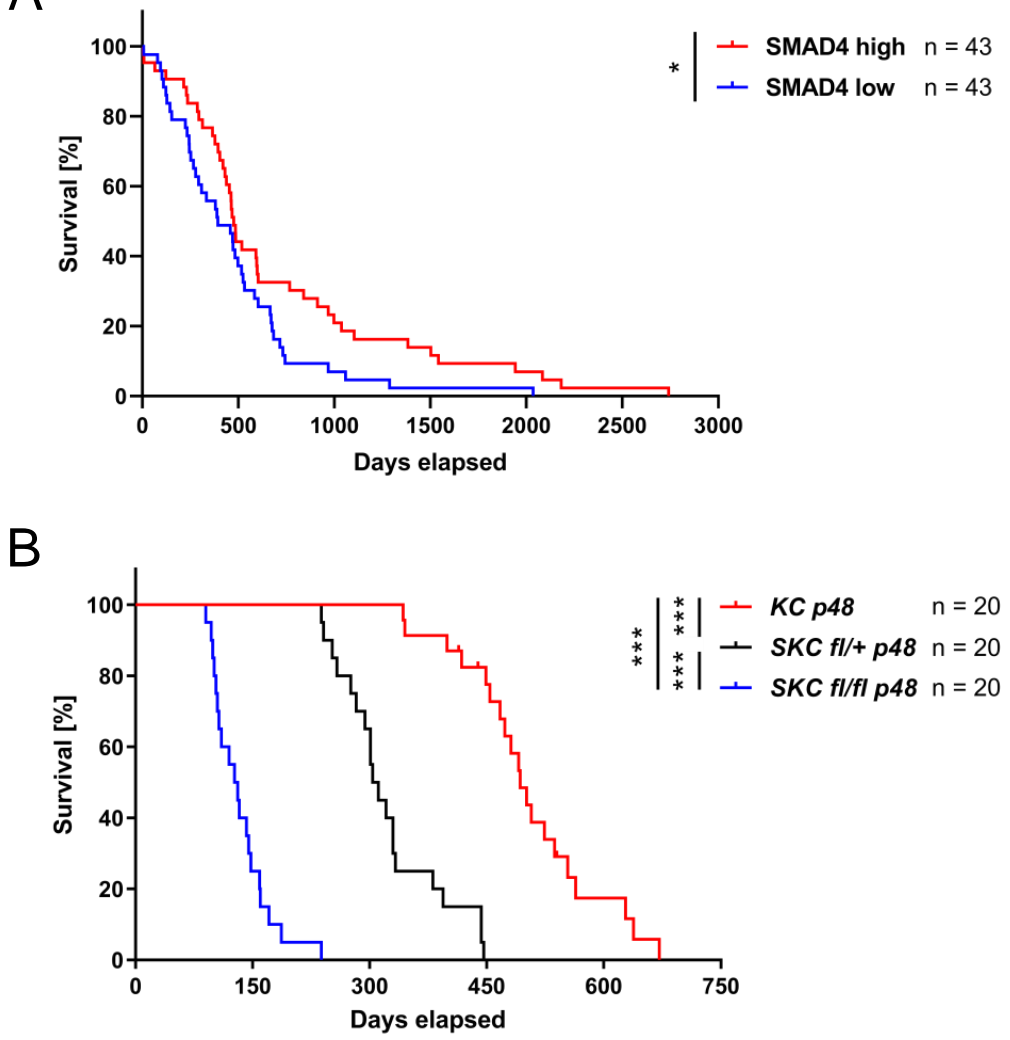

Figure 4 PDAC-associated survival is decreased upon Smad4 deficiency. A TCGA-derived patient data were analyzed according to SMAD4 status, considering the lower and upper percentiles (25:25) of SMAD4 signal. Patients associated with weak SMAD4 expression reveal a median survival of $394 \mathrm{~d}$ in contrast to $476 \mathrm{~d}$ in SMAD4 high expressing patients ( $\mathrm{n}=43$, respectively). B Animal survival studies were conducted with Smad4 WT, heterozygous Smad4 k.o. or homozygous Smad4 k.o. bearing GEMM of pancreatic cancer. The PDAC-associated median survival of 20 individuals per group is $493 \mathrm{~d}$ in $K C \mathrm{p} 48,307.5 \mathrm{~d}$ in $\mathrm{SKC} \mathrm{fl} /+\mathrm{p} 48$ and $129 \mathrm{~d}$ in $\mathrm{SKC} \mathrm{fl} / \mathrm{fl} \mathrm{p} 48$, respectively.

In addition to survival studies in aforementioned GEMMs, mice were sacrificed at 4 weeks, 8 weeks, 12 weeks and 6 months of age to investigate the formation of preneoplastic lesions and PDAC in detail. HE stainings illustrate gradual preneoplastic lesion development between 4 weeks and 12 weeks of age [Figure 6]. Already 4 weeks after birth, homozygous and heterozygous loss of Smad4 accelerates the formation of ADM and PanINs compared to KC p48 mice. Accordingly, the comparison of pancreas weight normalized to the corresponding body weight revealed significant differences inbetween 


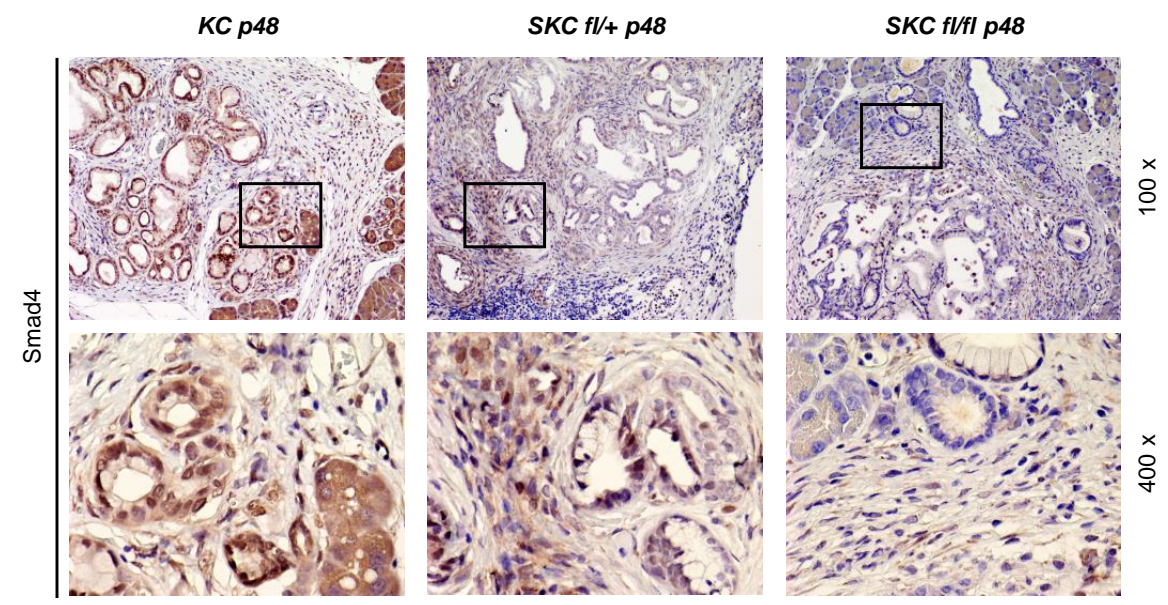

Figure 5 Homozygous Smad4 depletion abrogates epithelial Smad4 protein expression in the pancreas. Smad4 IHC demonstrated no reduction in staining of PanIN nuclei upon heterozygous Smad4 deficiency but complete loss in acini and preneoplastic lesions of $S K C \mathrm{fl} / \mathrm{fl} \mathrm{p} 48$ mice, while its expression was maintained in stromal cells.

genotypes from the age of 4 weeks onwards [Figure 7]. In each of the defined time points, the relative pancreas weight of $S K C$ fl/fl p48 mice was significantly increased compared to the Smad4 WT or heterozygous k.o. models, indicating tremendous acceleration of preneoplastic lesion formation upon homozygous Smad4 depletion in the context of oncogenic Kras activation. Importantly, PDAC formation occurred in the context of homozygous Smad4 depletion already at 8 weeks of age, with extensive desmoplasia leading to drastic gain of relative pancreas weight [Figure 7]. Pancreata of $S K C \mathrm{fl} /+\mathrm{p} 48$ mice significantly differed from KC p48 animals, demonstrating that partial loss of Smad4 was sufficient in driving PanIN and ADM formation beyond Smad4 WT controls. For SKC fl/fl p48 mice, first metastases in the liver were detectable at 12 weeks of age which is in line with $100 \%$ tumor incidence in 12 weeks old SKC fl/fl p48 animals [Figure 8, see number of included animals in Figure 7]. In contrast, age-matched $K C p 48$ and $S K C H /+p 48$ mice did not progress to PDAC at 12 weeks of age and show healthy liver tissue. For the majority of $S K C H /+p 48$ mice, tumor formation occurred between 12 weeks and 6 months of age, which is significantly earlier as in $K C$ p48 mice. The acceleration of PDAC formation upon complete loss 

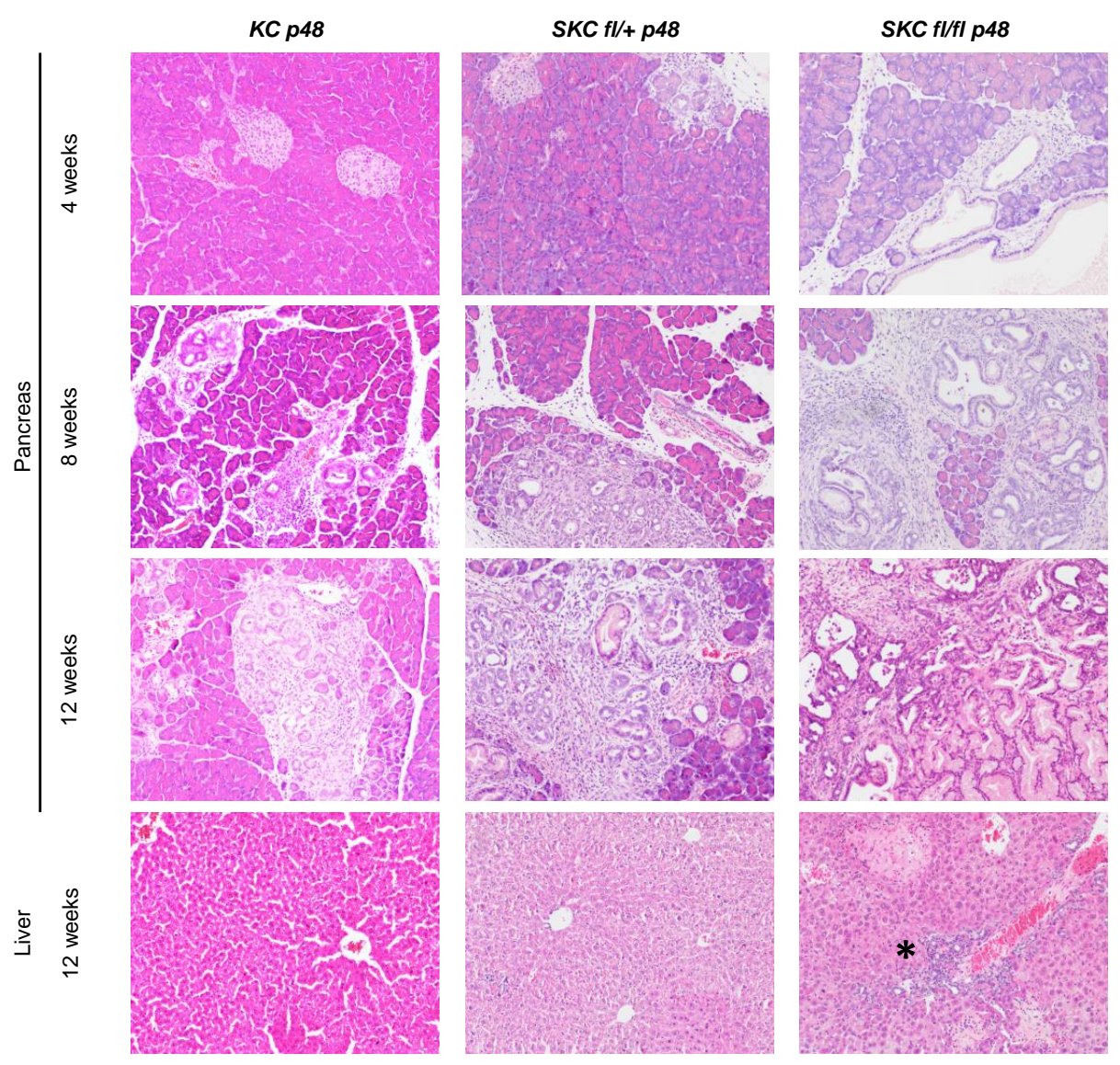

Figure 6 Smad4 deficiency accelerates formation of preneoplastic lesions and PDAC. HE stainings of 4 weeks, 8 weeks, and 12 weeks old mouse pancreata of the indicated genotypes reveal accelerated PanIN formation and progression upon loss of Smad4. Liver sections of 12 week old GEMM demonstrate early metastasis formation in $\mathrm{SKC} \mathrm{fl} / \mathrm{fl} \mathrm{p} 48$ mice [asterisk].

of Smad4 is in agreement with the observed significant differences in tumor-associated survival [Figure 4]. Importantly, only one SKC fl/fl p48 mice survived until 6 months of age, while others died prematurely due to severe tumor burden. SKC $\mathrm{Al} /+\mathrm{p} 48$ mice developed PDAC earlier than corresponding KC p48 animals, proposing the idea that already partial loss of Smad4 accelerated PDAC aggressiveness. This is in agreement with premature tumor-associated death of $S K C$ fl $/+p 48$ animals compared to Smad4 WT bearing $K C$ p48 mice. Kras WT expressing littermates of $K C \mathrm{p} 48, \mathrm{SKC} \mathrm{ll}+\mathrm{p} 48$, and $S K C$ fl/fl p48 did not show any significant differences in relative pancreas weight in the aforementioned defined time points [data not shown]. The combination of survival 

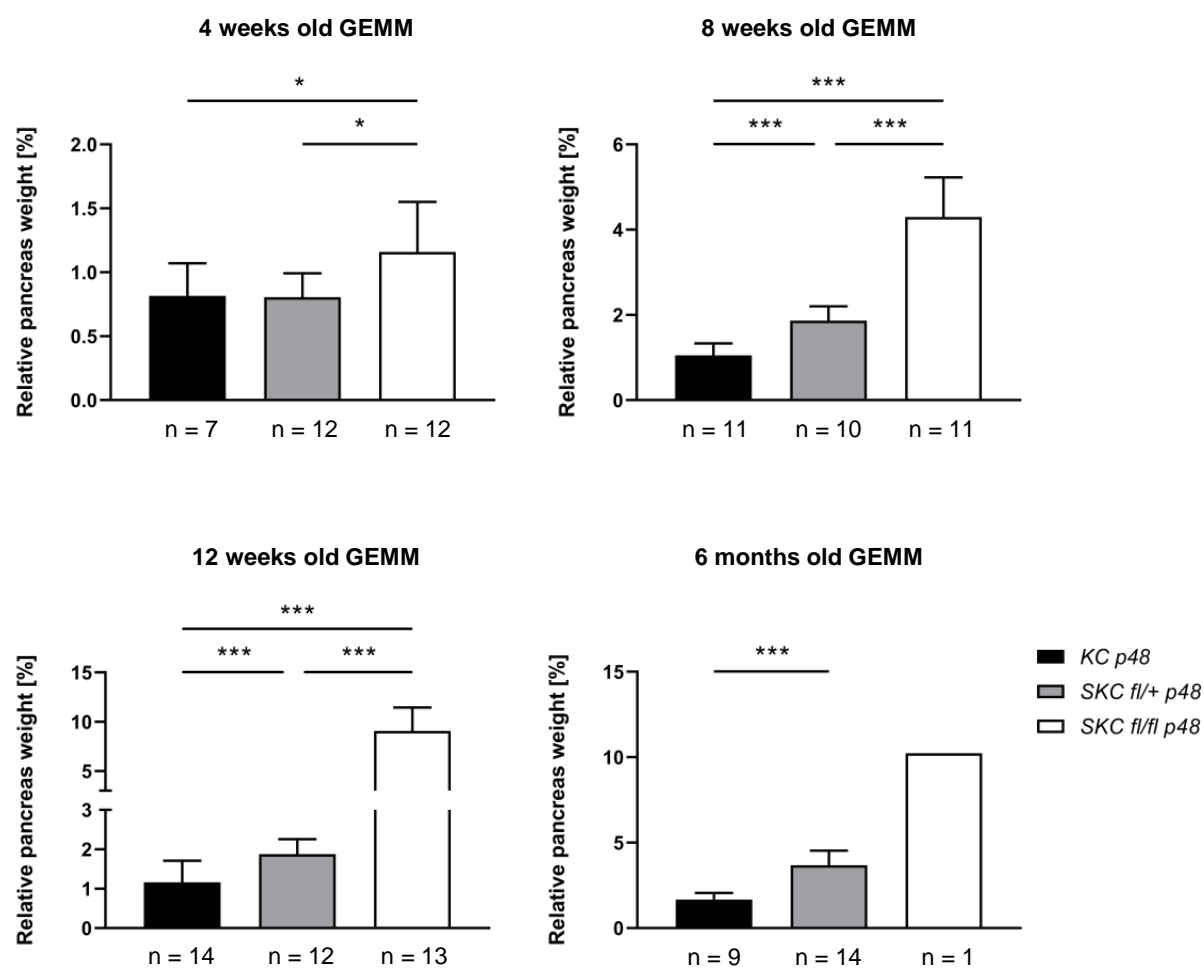

Figure 7 Smad4 deficiency accelerates gain of relative pancreas weight. Relative pancreas weight of indicated GEMMs is assessed by normalizing the pancreas weight to corresponding mouse body weight. Number of analyzed individuals is indicated for each defined time point.

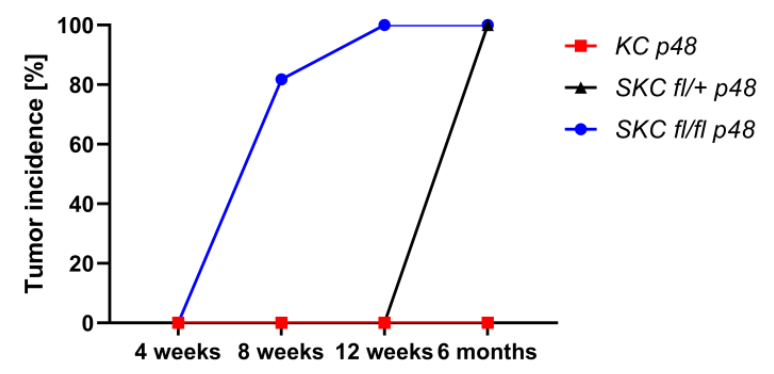

Figure 8 Smad4 deficiency hastens PDAC formation. PDAC development of indicated GEMMs was evaluated by analysing HE stainings of respective pancreata at the age of 4,8 , and 12 weeks and 6 months, respectively.

studies, analysis of relative pancreas weight, morphological assessment and evaluation of tumor incidence demonstrates that homozygous loss of Smad4 severely accelerates PDAC formation and PDAC-associated mortality in Kras-driven GEMMs. Moreover, 
partial Smad4-depletion also hastens tumorigenesis and accelerates PDAC-associated mortality beyond the $K C$ p 48 model, but not to the extent observed for $S K C$ fl/fl p48 mice.

A

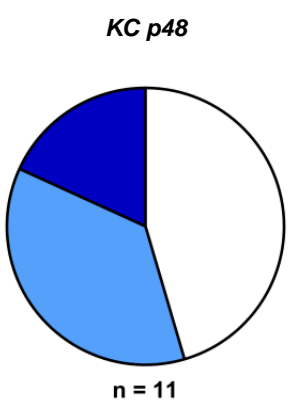

B

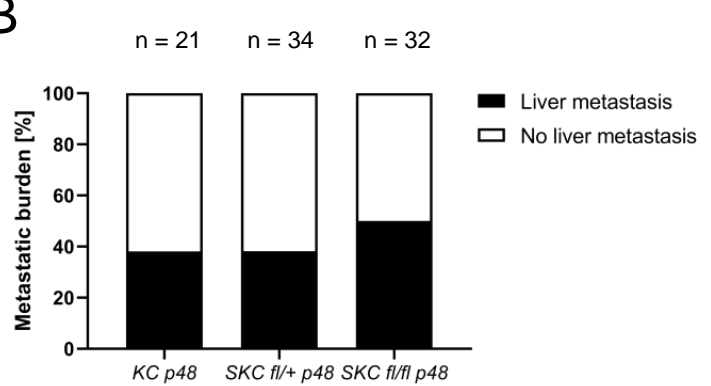

$S K C f l+p 48$

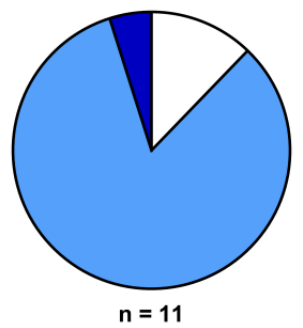

$\mathrm{C}$

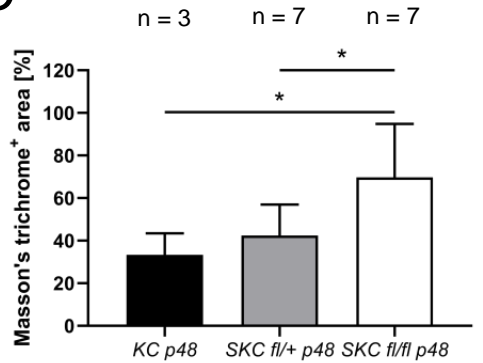

D

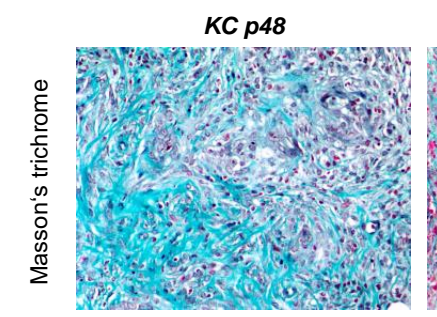
$S K C \mathrm{fl} / \mathrm{p}$ 48
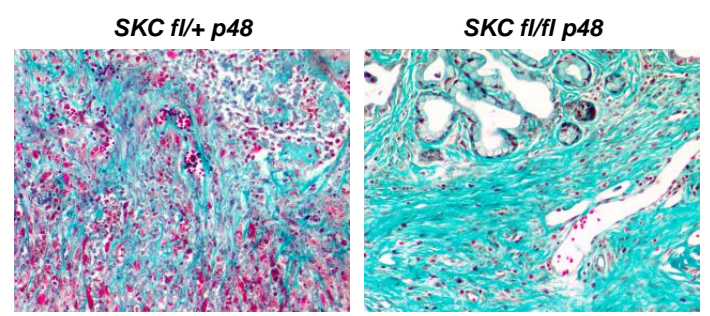

Figure 9 Homozygous loss of Smad4 favors metastasis formation in low grade PDAC. A Tumor stage was assed via evaluation of HE staining by Prof. P. Ströbel, Institute of Pathology, University Medical Center Göttingen. B Abundance of hepatic metastasis formation was evaluated using HE stainings in tumor-bearing animals of indicated genotypes. C Masson's trichrome staining for the evaluation of collagen deposition in tumor-bearing KC p48 $(\mathrm{n}=3), S K C \mathrm{fl} /+\mathrm{p} 48(\mathrm{n}=7)$ and SKC $\mathrm{fl} / \mathrm{fl}$ p 48 mice $(\mathrm{n}=7)$ was performed by Dr. med. H. Bohnenberger. Per animal, 10 pictures were taken at $200 \times$ and the positive fraction was normalized to total PDAC area in the corresponding picture. D Representative pictures of Masson's trichrome staining in the respective GEMM illustrate differences in PDAC architecture. 
In the next step, we aimed to figure out whether the extreme aggressiveness of Smad4depleted PDAC was reflected in the corresponding tumor stages. PDAC grading of tumor-bearing $K C$ p $48, S K C \mathrm{fl} /+\mathrm{p} 48$ and $\mathrm{SKC}$ fl/fl p48 mice was performed by Prof. P. Ströbel, Institute of Pathology, University Medical Center Göttingen [Figure 9 A]. Surprisingly, all SKC fl/fl p48-derived tumors met the criteria of low-grade G1 PDAC. In contrast, heterozygous Smad4 depletion favored the development of G2 tumors with reduced incidence of both G1 and G3 staged tumors. The established KC p48 model formed comparably more G1 and G3 tumors, thus representing the model system with the highest abundance of poorly differentiated PDAC. Accordingly, we checked for metastasis formation in HE-stained liver sections of all tumor-bearing GEMMs [Figure $9 \mathrm{~B}]$. In agreement with the observed gain of PDAC aggressiveness upon homozygous Smad4 depletion, the metastatic burden was increased in the $S K C$ fl/fl p48 model compared to the others. This is of particular interest as the life span of SKC $\mathrm{fl} / \mathrm{fl}$ p48 mice was up to 2 to 3 times shorter than $K C p 48$ and $S K C H /+p 48$ mice employed in this analysis. These contradictory data imply that not the tumor differentiation as evaluated by tumor grading but rather the PDAC architecture is the crucial factor in metastatic spread. To investigate differences in tumor composition, we analyzed collagen abundance as visualized by Masson's trichrome staining performed by Dr. med. H. Bohnenberger, Institute of Pathology, University Medical Center Göttingen [Figure $9 \mathrm{C}, \mathrm{D}]$. Here we identified significantly increased collagen deposition upon homozygous Smad4 depletion as compared to heterozygous loss of Smad4 and Smad4 WT in the context of oncogenic Kras activation. Although $S K C \mathrm{fl} /+\mathrm{p} 48$ mice developed comparably higher PDAC stages corresponding to rather dedifferentiated tumors, the metastasis formation remained at the same level as in $K C$ p48 mice, demonstrating that partial Smad4 depletion was insufficient in driving processes leading to different tumor structure and promoting metastasis. 


\subsubsection{NFATc1 and Smad3 cooperate in Smad4 deficiency}

Apart from phenotypic analyses of GEMMs bearing Smad4 WT, heterozygous Smad4 k.o. or homozygous Smad4 depletion in the context of oncogenic Kras activation, we aimed to focus on Nfatc1/Smad3 cooperativity in the aforementioned models. IF staining in SKC fl/fl p48-derived PDAC tissue revealed colocalization of respective Nfatc1 and Smad3 signals [Figure 10]. Smad3 was detected preferably in acini, PanIN lesions, tumor cells and immune cells. Strong Nfatc1 signal, however, was spotted in immune cells and potential invading cancer cells, respectively. Embedded in tumor stroma, NFATc1 and Smad3 double positive cells were identified [see arrowheads in Figure 10]. However, simultaneous Nfatc1 and Smad3 expression is a prerequisite for the existence of Nfatc1/Smad3 complexes which needed to be validated in further experiments.

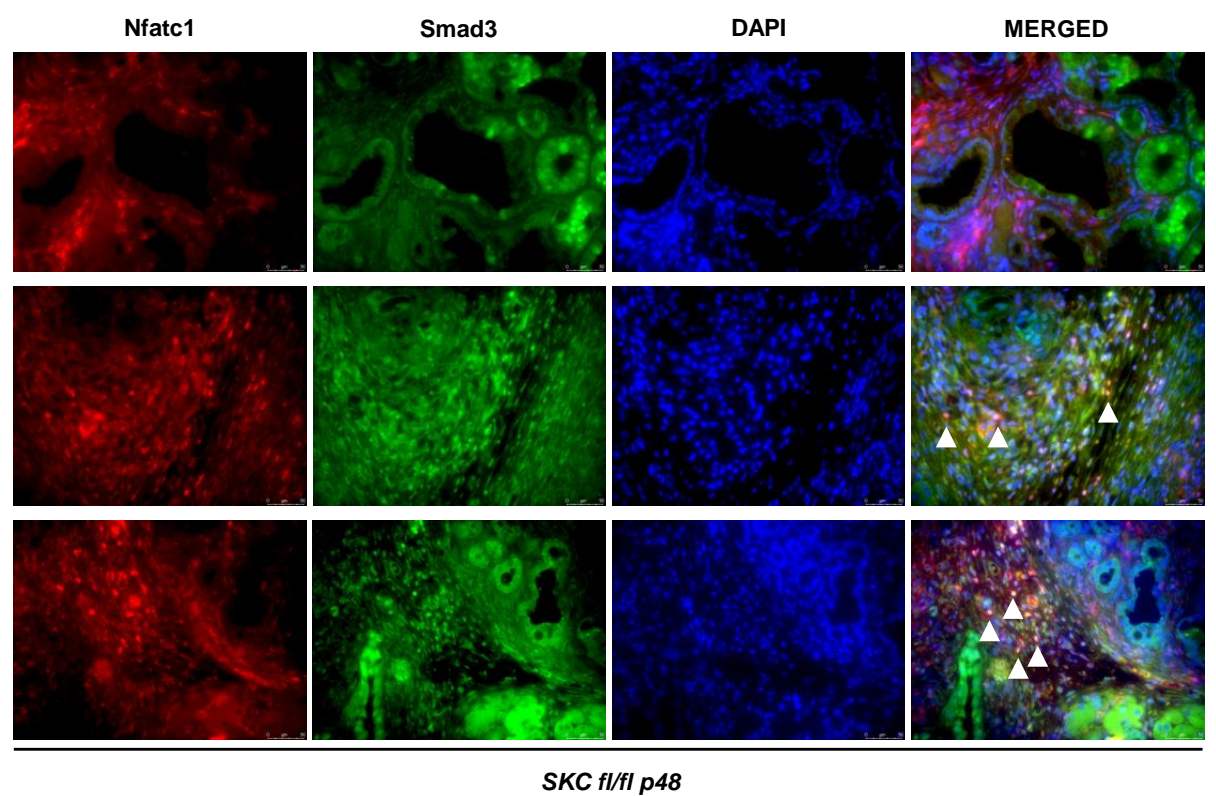

Figure 10 Nfatc1/Smad3 double positive tumor cells in Smad4 deficiency. Pancreatic tissue containing healthy acinar cells, PanIN lesions and differentiated PDAC was stained for Nfatc1 (red) and Smad3 (green) via IF. Arrowheads mark double positive cells surrounded by tumor stroma. Blue DAPI staining highlights nuclei. Magnification is set at $400 \mathrm{x}$. 


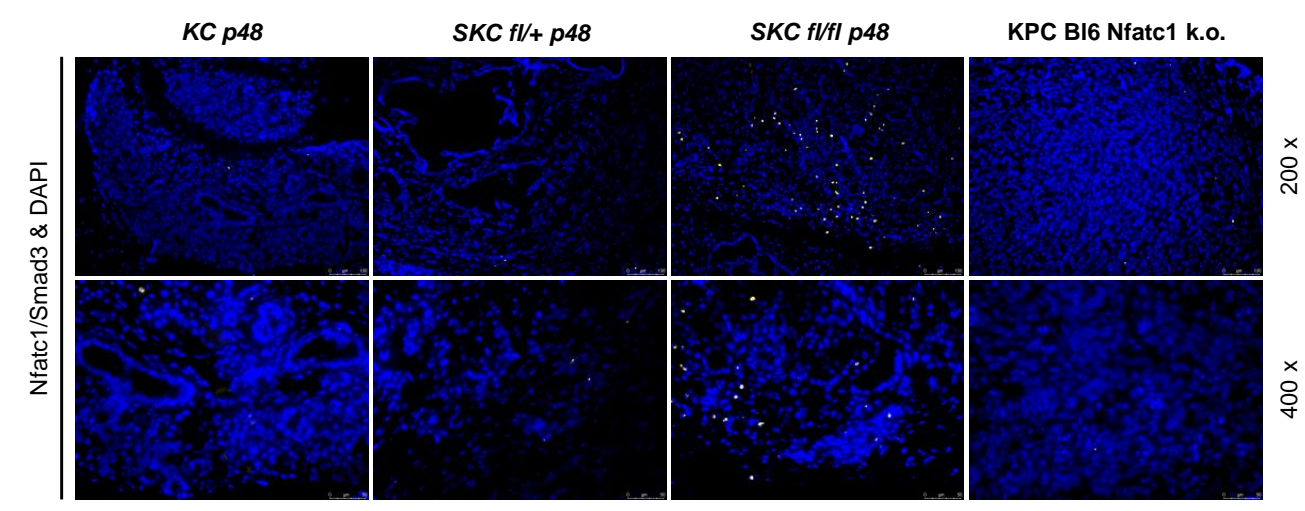

Figure 11 Nfatc1/Smad3 complexes in PDAC and immune cells. PDAC tissue of $K C \mathrm{p} 48, \mathrm{SKC} \mathrm{fl} / \mathrm{p} 48, \mathrm{SKC} \mathrm{fl} / \mathrm{fl} \mathrm{p} 48$, and KPC Bl6 orthotopically transplanted tumor cells were utilized for an in situ PLA assay. Yellow signal indicates Nfatc1/Smad3 complexes while nuclei are counterstained with DAPI.

To investigate Smad4-dependent Nfatc1/Smad3 complex formation using the endogenous expression levels, we performed in situ fluorescence-based PLA studies on PDAC tissue derived from $K C \mathrm{p} 48, \mathrm{SKC} \mathrm{fl}+\mathrm{p} 48$ and $\mathrm{SKC}$ fl/fl p48 mice, respectively [Figure 11]. As a negative control, we processed PDAC tissue from KPC Bl6 Nfatc1 k.o. orthotopically transplanted tumor cells which was provided by D. Spyropoulou, Department of Gastroenterology and Gastrointestinal Oncology, University Medical Center Göttingen as part of her medical doctoral thesis. Based on close proximity of Nfatc1 and Smad3, rolling circle DNA synthesis of oligonucleotide-bearing secondary antibodies is exploited to visualize Nfatc1/Smad3 complexes via fluorescence-labeled DNA probes. Nuclear staining by DAPI was used to evaluate the cellular location of observed Nfatc1/Smad3 complexes. In the $K C \mathrm{p} 48$ and $\mathrm{SKC} \mathrm{H} /+\mathrm{p} 48$ model, there was occasional detection of Nfatc1/Smad3 complexes in cells with small circular nuclei detached from epithelial structures, hinting towards nuclear Nfatc1/Smad3 complexes in immune cells. The most frequent occurrence of Nfatc1/Smad3 signal was identified in $S K C$ fl/fl p48 PDAC. As observed for the IF staining in the same tumor, we propose nuclear Nfatc1/Smad3 complex formation in invading tumor cells and immune cells, respectively, while there was no detection in preneoplastic lesions. In agreement with $K C$ p48 and $S K C H /+p 48$-derived tissue, Nfatc1/Smad3 complexes were 
detected in the periphery of epithelial structures and within comparably more dedifferentiated PDAC. In our KPC Bl6 Nfatc1 k.o. control, we recognized weak signal of Nfatc1/Smad3-bearing host-derived immune cells in the anaplastic tumor, thereby validating the observations made in the other tissues.

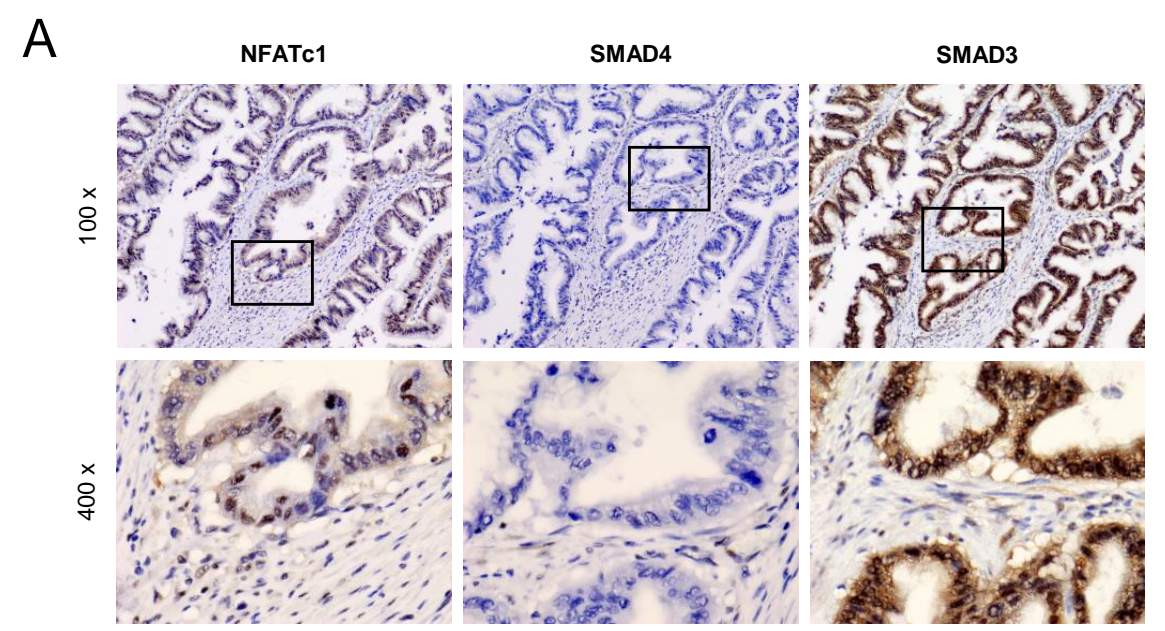

B

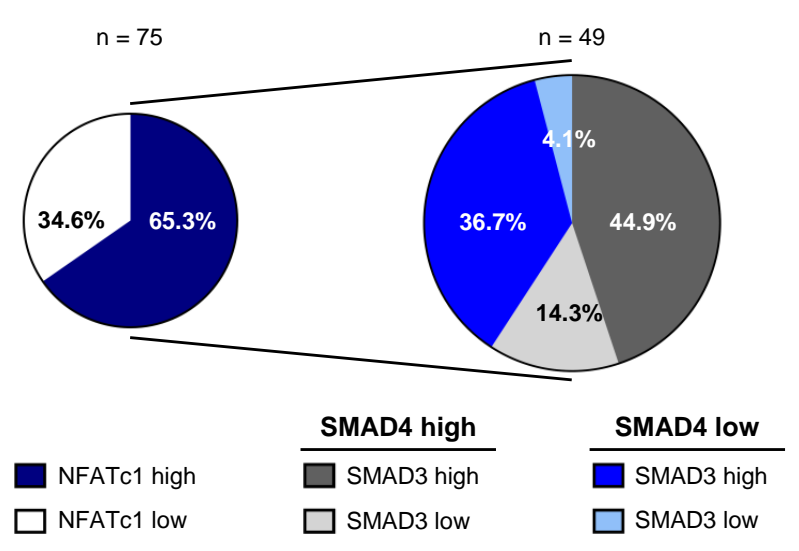

Figure 12 NFATc1 cooperates with SMAD3 in SMAD4-deficient human PDAC. A Exemplary IHC stainings of NFATc1, SMAD4, and SMAD3 in human PDAC tissue. B Stratification according to NFATc1, SMAD4, and SMAD3 expression and localization in the tumor cell as assessed by IHC in human TMA.

Finally, we aimed to confirm the prevalence of nuclear NFATc1 signal combined with robust nuclear SMAD3 expression in patient-derived PDAC tissue in the context of SMAD4 deficiency. Wilentz et al. (2000b) and Liszka (2014) described IHC as a reliable 
tool to assess the SMAD4 status of cells. Both loss of heterozygosity (LOH) subsequent to truncation or depletion of one allele leads to a complete loss of staining. Meanwhile, unaltered immune cells and fibroblasts serve as SMAD4 staining controls. Nuclear localization of NFATc1 and SMAD3 was interpreted as a measure for transcriptional activity [Figure $12 \mathrm{~A}$ ]. Of all analyzed patient-derived PDAC samples $(\mathrm{n}=75)$, two thirds stained positive for nuclear NFATc1 [Figure $12 \mathrm{~B}$ ]. This patient cohort was further analyzed according to SMAD4 status, revealing $40.8 \%$ of SMAD4-depleted patient tissues. Regardless of SMAD4 status, in this patient collective, SMAD3 was preferably situated in the nucleus, with a tendency of increased SMAD3 activation in the context of SMAD4 deficiency. The tissue microarray (TMA) analysis of human PDAC confirmed the existence of a patient subgroup with strong nuclear accumulation of NFATc1 and SMAD3 in the context of SMAD4 depletion (36.7\%).

Via IF and in situ PLA approaches, we confirmed the existence of nuclear Nfatc1/Smad3 cooperativity and complex formation predominantly in the context of homozygous Smad4 depletion. However, if and to what extend Nfatc1/Smad3 complex-driven transcription is contributing to the extreme acceleration of PDAC progression in the SKC fl/fl p48 model remains a matter of investigation. Phenotypic analyses suggest that heterozygous loss of Smad4 is already a sufficient promoter of preneoplastic lesion formation and PDAC establishment leading to reduced survival of $\mathrm{SKC} \mathrm{fl} / \mathrm{p} 48$ mice compared to $K C$ p48. Detailed molecular analyses in all three GEMMs are limited by weak endogenous Nfatc1 protein expression, suggesting the introduction of additional experimental systems to elucidate if and under which circumstances the inflammatory transcription factor Nfatc1 interacts with Smad3 for joint regulation of gene expression. 


\subsubsection{Smad4 deficiency favors nuclear NFATc1/Smad3 complex assembly}

For an in-depth analysis of conditions driving or disrupting nuclear NFATc1/Smad3 transcription complex assembly, we switched to another model bearing NFATc1 overexpression. Therefore, we relied on primary murine PDAC cells derived from NKC p48 mouse model introduced by Baumgart et al. (2014). Those NKC-II cells are characterized by constitutive nuclear HA-tagged NFATc1 along with oncogenic Kras activation causing a dramatic acceleration of tumorigenesis and thus mimicking the aggressive nature of human PDAC (Baumgart et al., 2014). Importantly, those NKC p48-derived primary PDAC cells maintained Smad4 WT status and remained responsive to TGF $\beta$ treatment (Hasselluhn et al., in revision).

Given that IF and PLA experiments in GEMMs with different Smad4 states suggested Nfatc1/Smad3 complex formation preferably in the context of Smad4-depleted PDAC, we further aimed at investigating NFATc1/Smad3 complex formation in a Smad4dependent manner. Hence, we applied CRISPR/Cas9-mediated genome editing by transfecting two corresponding sgRNA sequences targeting exon 3 of the Smad4 gene. Subsequent GFP-guided single clones sorting by FACS and clonal growth led to the establishment of NKC-II Smad4 k.o. cell line. As a control, we picked another clone which underwent the same procedure and selection pressure but maintained its Smad4 WT status as confirmed by sequencing [data not shown]. Based on genomic DNA extracted from parental cells and CRISPR/Cas9 clones, targeted PCR for the Smad4 locus was performed. The PCR product was separated on an agarose gel to visualize the loss of $550 \mathrm{bp}$ in the Smad4 gene [Figure $13 \mathrm{~A}$ ]. Loss of exon 3 introduces a frameshift into the nucleotide sequence, leading to truncation and misfolding of the Smad4 protein. Accordingly, we checked for Smad4 protein expression via western blot and further verified the integrity of TGF $\beta$ signaling in Smad4 WT and -deficient clones [Figure 13 B]. Importantly, we aimed to preserve unaltered TGF $\beta$ response in the CRISPR/Cas9 clones for examining its role in potential NFATc1/Smad3 complex formation. While 


\section{Results}

A

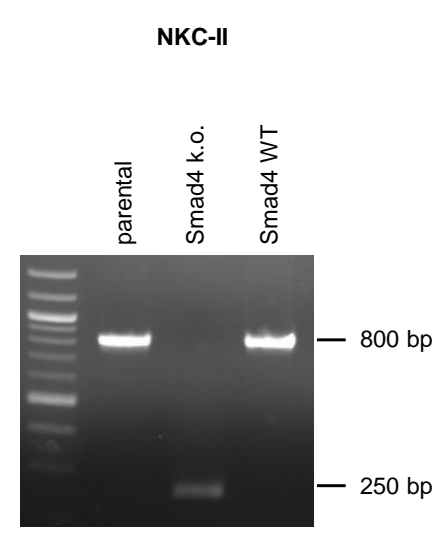

C

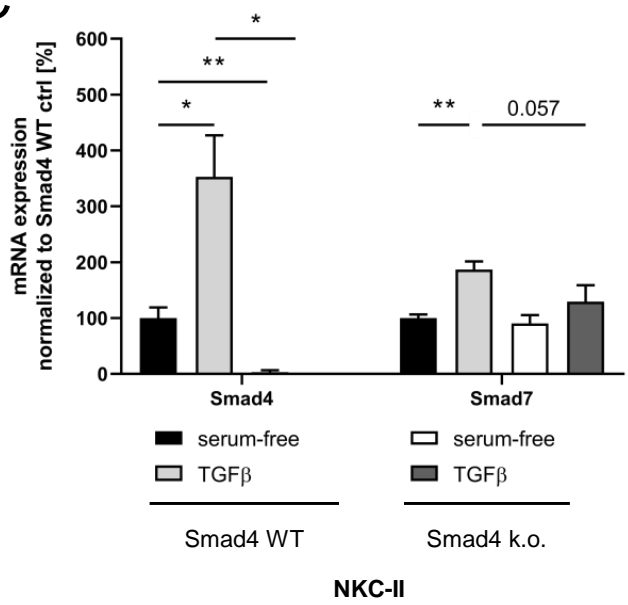

B

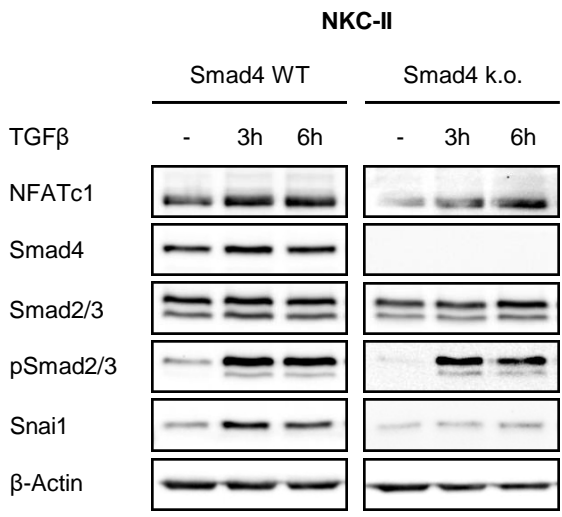

NKC-II: murine; Kras ${ }^{\mathrm{G} 12 D}$; NFATc1-HA c.n.

Figure 13 Establishment of CRISPR/Cas9-mediated Smad4 knock out in NKC-II cells. A Genomic DNA of parental NKC-II cells and two CRISPR/Cas9 clones PCR of Smad4 locus using primer pair P1 separated by an agarosel gel. B Western blot analysis of indicated proteins demonstrating response to TGF $\beta[10 \mathrm{ng} / \mathrm{ml}]$ and successful Smad4 depletion. C QRT-PCR of selected genes indicating alterations in $\mathrm{TGF} \beta$ response $[10 \mathrm{ng} / \mathrm{ml}, 24 \mathrm{~h}]$.

Smad4 expression was completely depleted in NKC-II Smad4 k.o. cells, it remained intact in Smad4 WT cells. Regardless of Smad4 status, cells responded to TGF $\beta$ treatment with subsequent phosphorylation of recepter Smads. Snai1, a transcriptional target of Smad4, was induced by TGF $\beta$ in the Smad4 WT clone, but failed to be induced in the absence of Smad4. On mRNA level [Figure $13 \mathrm{C}$ ], we confirmed the integrity of TGF $\beta$ response by consequent induction of the inhibitory Smad (Smad7) 
and the loss of Smad4 expression. We hereby confirmed successfull CRISPR/Cas9mediated Smad4 k.o. in murine primary NKC-II cells on DNA, mRNA and protein level and demonstrated unbiased TGF $\beta$ response in both NKC-II Smad4 WT and k.o. clones.

Characterizing the NKC-II clones comprised detailed analysis of functional consequences of TGF $\beta$ response depending on the Smad4 status. As TGF $\beta$ is a known accelerator of migration (Ciacci et al., 1993; Mythreye \& Blobe, 2009; Takai et al., 2012; Gao et al., 2014; Melzer et al., 2017), we assessed TGF $\beta$-mediated migration in a wound healing assay using live cell imaging [Figure $14 \mathrm{~A}$ ]. Regardless of Smad4 expression, TGF $\beta$ treatment hastened the intrinsic migration speed in both clones. Interestingly, the maximal migration distance covered by NKC-II Smad4 k.o. cells significantly exceeded the Smad4 WT clone, ultimately leading to faster wound closure. To figure out if that phenomenon was indeed an increased migrative capacity fostered by TGF $\beta$ or a side effect of propagated cell growth, we determined the proliferative capacity of CRISPR/Cas9 clones via cell counting [Figure 14 B]. In the observed timeframe, there were no significant differences in proliferation detectable according to Smad4 status. Importantly, this was confirmed by comparing CRISPR/Cas9 clones of two additional PDAC cell lines [see Appendix Figure 32].

In addition, we aimed to identify the Smad4 clone's ability to anchorage-independent growth depending on TGF $\beta$ [Figure $14 \mathrm{C}$ ]. Essentially, Smad4 deficiency alone significantly triggered colony formation in the soft agar assay. Additionally, TGF $\beta$ administration further promoted clonal growth of NKC-II Smad4 k.o. cells while there was no effect on Smad4 WT cells. Anchorage-independent growth is a hallmark of carcinogenesis and is considered a characteristic feature of stem cells (Taddei et al., 2012). Therefore, we assessed the stem cell capacity of both NKC-II clones via sphere formation assay using the final sphere number and size distribution as readouts [Figure $14 \mathrm{D}]$. TGF $\beta$ treatment led to decreased sphere numbers in both clones, with NKC-II Smad4 k.o. cells forming slightly more spheres compared to Smad4 WT. Importantly, 
the size of spheres differed extremely between clones. While Smad4 WT cells formed small spheres that were turning bigger upon TGF $\beta$ application, NKC-II Smad4 k.o. cells rather developed huge spheres independent of TGF $\beta$. With the help of diverse
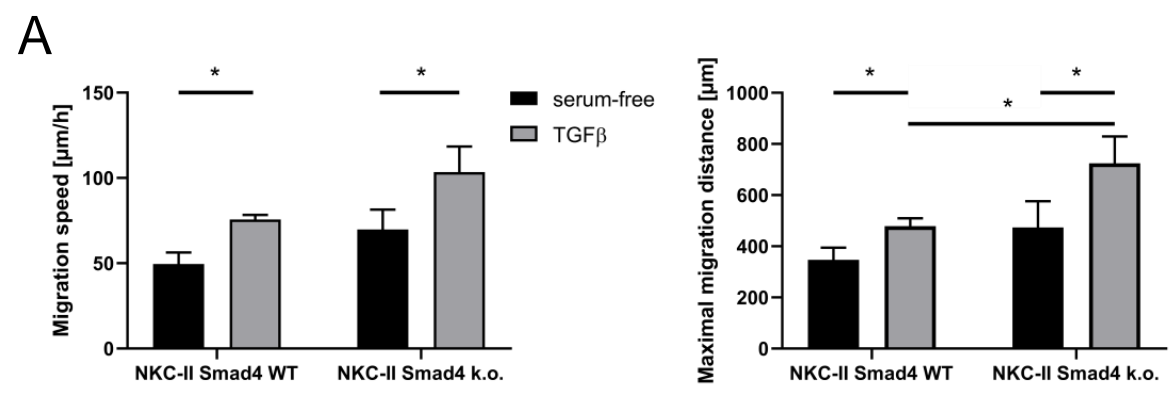

B

C
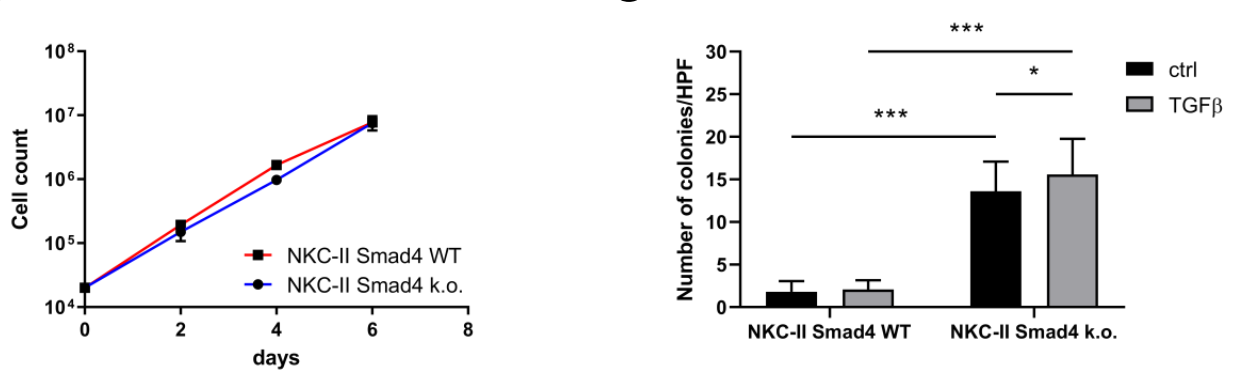

D
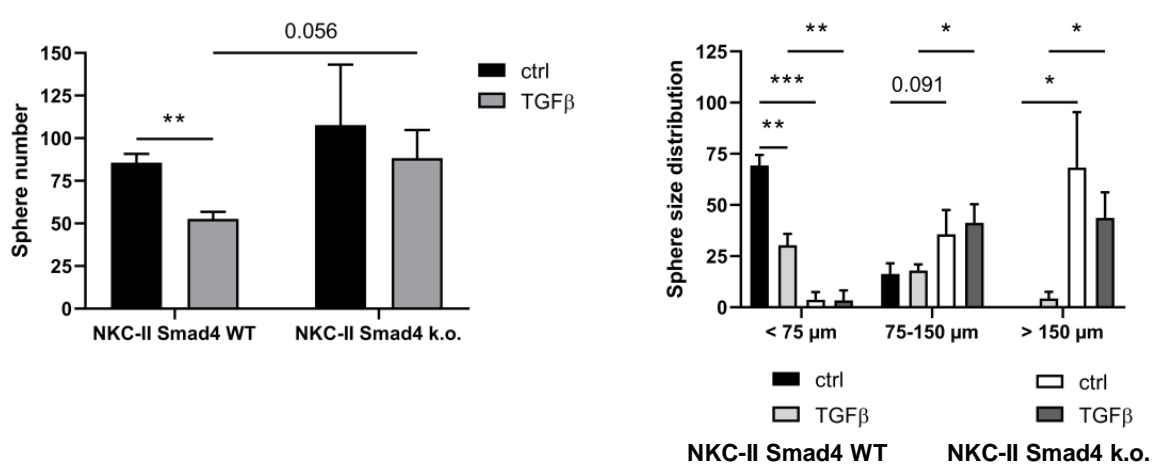

Figure 14 Smad4-dependent functional consequences of TGF $\beta$ treatment. TGF $\beta$ was administered as $10 \mathrm{ng} / \mathrm{ml}$. A Wound healing assay in confluent, serum-starved NKC-II clones $(\mathrm{n}=3)$. Migration was retraced via live cell imaging by taking one picture per hour. B Proliferative capacity was measured by cell counting every second day $(\mathrm{n}=$ 3). C Anchorage-independent growth was assessed via colony counting in soft agar assay $7 \mathrm{~d}$ after seeding $(\mathrm{n}=3)$. D Sphere formation in low attachment plates was evaluated after $7 \mathrm{~d}$ cultivation $(\mathrm{n}=3)$ by counting and diameter measurement. 
functional assays we identified increased migrative and stem cell abilities in Smad4depleted NKC-II cells which were only partly determined by TGF $\beta$ treatment. In contrast, Smad4 deficiency has no effect on tumor cell proliferation.

A

NKC-II Smad4 WT

NKC-II Smad4 k.o.
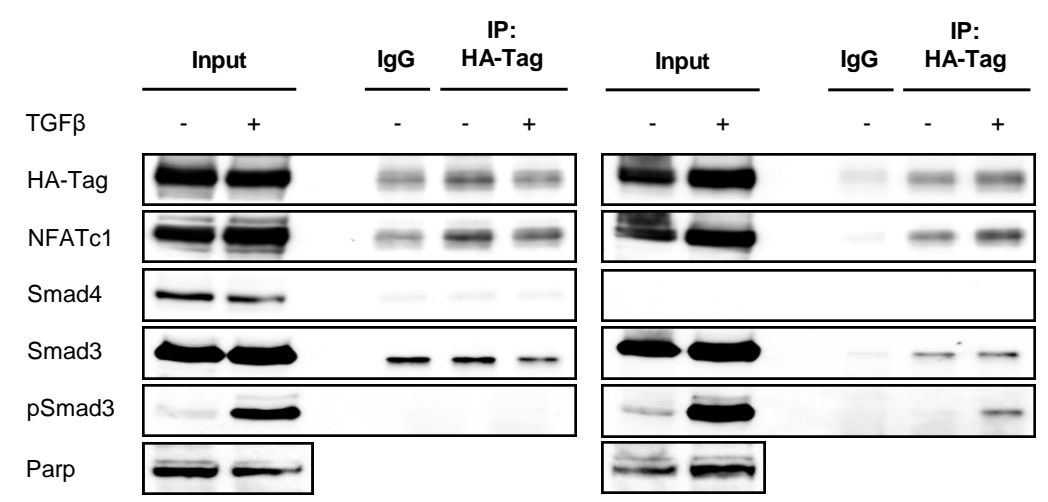

NKC-II: murine; KrasG12D; NFATc1-HA c.n.

$\mathrm{B}$
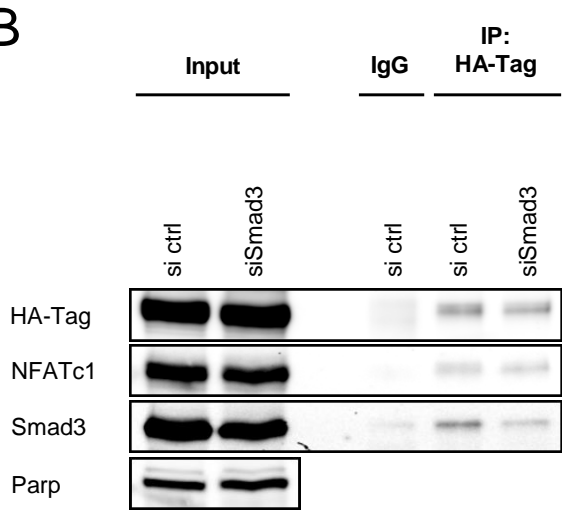

NKC-II Smad4 k.o.: murine; Kras ${ }^{\mathrm{G} 12 \mathrm{D}} ;$ NFATc1-HA c.n.
C

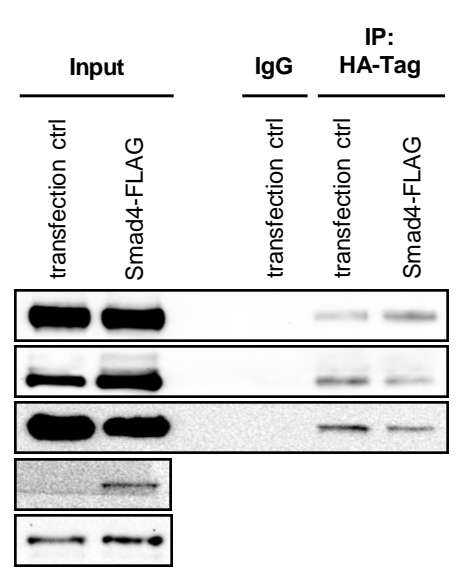

NKC-II Smad4 k.o.: murine; Kras ${ }^{\mathrm{G} 12 \mathrm{D}}$; NFATc1-HA c.n.

Figure 15 Smad4 dependency of nuclear NFATc1/Smad3 complex formation. Co-IP experiments were conducted with nuclear lysates of indicated cell lines utilizing HA-tagged NFATc1 as bait and confirming attached proteins via western blot. A Co-IP experiment including both NKC-II clones subsequent to $24 \mathrm{~h}$ TGF $\beta$ exposition or serum starvation only. B Knockdown of Smad3 confirms signal specificity of Smad3 bands in co-IP experiments. C Reexpression of Smad4 in NKC-II Smad4 k.o. cells intervenes with NFATc1/Smad3 complex formation. 
To this point, we observed functional differences in NKC-II cells upon Smad4 deficiency. Further, we utilized these cells to investigate NFATc1/Smad3 complex formation and aimed to elucidate whether this transcription complex relies on the Smad4 status or active TGF $\beta$ signaling, respectively. Therefore, we isolated nuclear extracts of NKC-II Smad4 WT and k.o. cells to check for nuclear complex formation via co-IP utilizing the HA-tagged NFATc1 as bait [Figure 15 A]. In Smad4 WT expressing cells, detected Smad3 and Smad4 signal in co-IP samples was similar to measured background determined by IgG. Successful activation of the TGF $\beta$ pathway was controlled via pSmad3 detection in the input samples of both clones, respectively. However, only NKC-II Smad4 k.o. lysates presented a strong signal of co-immunoprecipitated pSmad3 upon TGF $\beta$ administration. Additionally, we performed a siRNA-mediated Smad3 knock down with subsequent nuclear lysate isolation and co-IP to verify signal specificity of the Smad3 protein band detection [Figure 15 B]. Indeed, we noticed strong reduction of co-immunoprecipitated Smad3 levels upon introduction of Smad3 silencing. To confirm the observed Smad4 dependency of NFATc1/Smad3 complex formation, a fulllength FLAG-tagged Smad4 construct was reintroduced into NKC-II Smad4 k.o. cells [Figure $15 \mathrm{C}$ ]. Nuclear lysates validated successful Smad4 protein expression in line with reduced signal of co-immunoprecipitated Smad3 in FBS-supplemented cultivation medium. By using nuclear lysates of NKC-II clones in different co-IP approaches and in line with data from our transgenic mouse model, we identified NFATc1/Smad3 complex formation preferentially in Smad4-deficient PDAC cells.

In our first co-IP experiments [Figure $15 \mathrm{~A}$ ], we observed that TGF $\beta$ administration fostered Smad3 phosphorylation and consequently promoted its complex formation with NFATc1. However, NFATc1/Smad3 complexes were not strictly depending on TGF $\beta$ as they also occurred in serum-deprived cells. In order to further explore if TGF $\beta$ influences NFATc1/Smad3 complex formation, we isolated nuclear lysates of NKC-II Smad4 k.o. cells that were treated with increasing concentrations of TGF $\beta$ for $24 \mathrm{~h}$ subsequent to serum starvation [Figure 16]. Co-IP experiments revealed an increase of co-immunoprecipitated Smad3 in the presence of active TGF $\beta$ signaling 


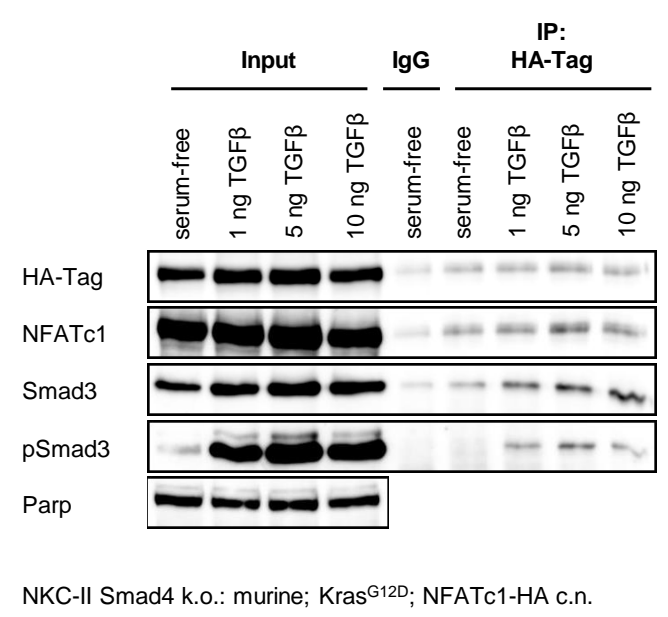

Figure 16 TGF $\beta$ dependency of nuclear NFATc1/Smad3 complex formation. NKC-II Smad4 k.o. cells were serum-starved for $24 \mathrm{~h}$ prior to administration of indicated TGF $\beta$ concentrations for another $24 \mathrm{~h}$. Nuclear lysates were conducted to co-IP utilizing the HA-tagged NFATc1. Complex partners were confirmed by western blot for indicated targets.

accompanied by a gradual increase of Smad3 phosphorylation. We demonstrated that the complex assembly itself was not relying on TGF $\beta$ activity, but indicated that it supported an increase in NFATc1/Smad3 complex formation.

With the establishment of Smad4 k.o. cells via CRISPR/Cas9-mediated genome editing in NKC-II cells, we accomplished to create a PDAC model system relying on the same genetic background, thereby excluding artifacts of PDAC heterogeneity. This enabled us to study Smad4 effects in cells with unaltered TGF $\beta$ response, revealing advances in migration, anchorage-independent growth, and sphere formation upon Smad4 deficiency. Importantly, we demonstrated NFATc1/Smad3 nuclear complex formation in Smad4-depleted NKC-II cells that is abrogated upon reintroduction of Smad4. NFATc1/Smad3 complex assembly occurred irrespective of TGF $\beta$ administration.

Next, we aimed to investigate whether Nfatc1/Smad3 complex assembly was limited to murine models of PDAC. To this end, we investigated Nfatc1/Smad3 complex formation in established human PDAC cell lines [Figure 17]. Co-IP studies in BxPC3 
A

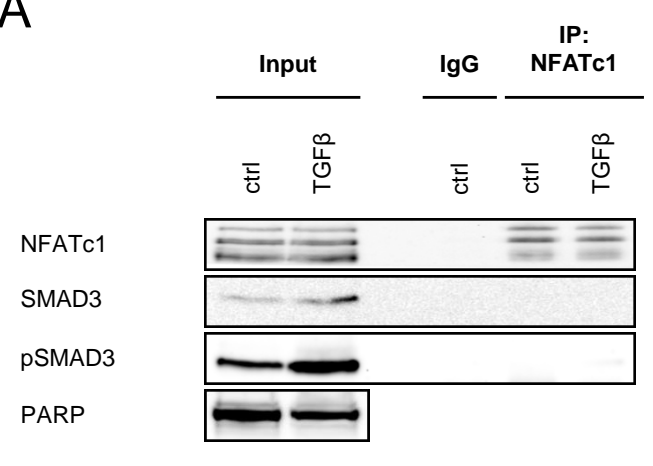

BxPC3: human; KRAS WT; SMAD4 HD

C

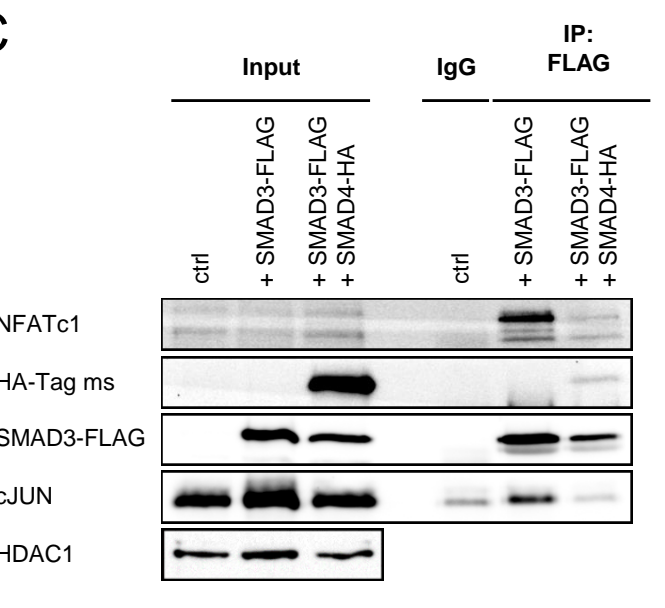

B

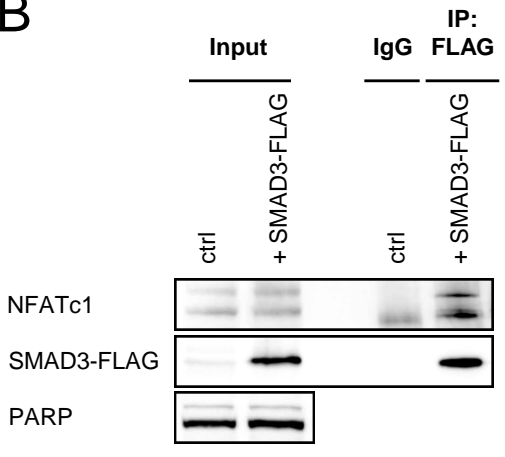

PaTu8988t: human; KRASG12D; SMAD4 HD

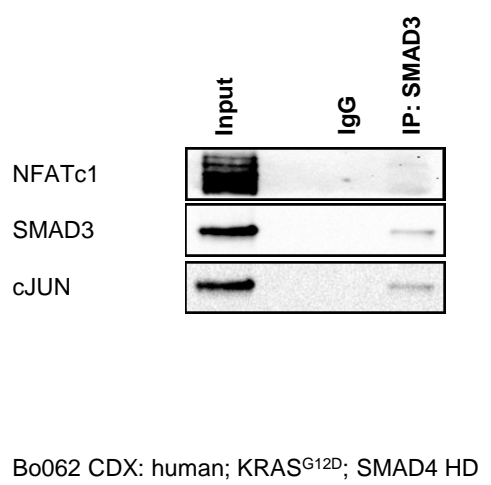

Figure 17 NFATc1/SMAD3 complex assembly depends on SMAD4 deficiency and oncogenic KRAS activation. A SMAD4-depleted human BxPC3 cells were serum-starved prior to $24 \mathrm{~h}$ TGF $\beta$ treatment. Nuclear lysates were conducted to co-IP studies utilizing endogenous NFATc1 protein expression and visualized via western blot. B SMAD4-depleted human PaTu8988t cells were transfected with FLAG-tagged SMAD3 construct $24 \mathrm{~h}$ before isolation of nuclear fraction. FLAG-based co-IP experiments displayed enrichment of coimmunoprecipitated endogenous NFATc1 protein in western blot. C PaTu8988t cells were transfected with FLAG-tagged SMAD3 \pm HAtagged SMAD4 $24 \mathrm{~h}$ prior to nuclear lysate extraction. Co-IP studies utilizing the FLAG-tag checked for NFATc1/SMAD3 complex formation via western blot. D Primary human PDAC cells isolated from a patient-derived xenograft were harvested for nuclear lysates. Subsequent co-IP experiments using endogenous SMAD3 as bait were displayed via western blot analysis. 
cells did not reveal co-immunoprecipitated Smad3 or pSmad3 bound to Nfatc1 despite homozygous SMAD4 deletion [Figure $17 \mathrm{~A}$ ]. As BxPC3 cells retain KRAS WT status, we concluded that oncogenic KRAS activation is a prerequisite for Nfatc1/Smad3 complex assembly. We next employed SMAD4-deficient PaTu8988t cells to study Smad3 complex formation by introducing a FLAG-tagged SMAD3 construct into the human PDAC cells. Via co-IP studies with subsequent western blotting, we visualized accumulation of endogenous NFATc1 protein bound to SMAD3-FLAG [Figure $17 \mathrm{~B}$ ]. Importantly, by co-transfection of FLAG-tagged SMAD3 and HA-tagged SMAD4, we could demonstrate abrogation of co-immunoprecipitated NFATc1 bound to SMAD3, confirming SMAD4 deficiency as a prerequisite for NFATc1/SMAD3 complex assembly [Figure $17 \mathrm{C}$ ]. Ultimately, the question remained whether NFATc1/SMAD3 complexes assemble in human patient tissue. Therefore, we chose a primary human PDAC cell line which was expanded from patient-derived xenograft studies as part of a collaboration with Prof. S. Hahn, Clinical Research Center, Ruhr-Universität Bochum and Prof. J. Siveke, West German Cancer Center, University Hospital Essen [Figure 17 D]. Corresponding sequencing data gathered in cooperation with the Institute of Human Genetics, University Medical Center Göttingen, confirmed oncogenic KRAS activation and homozygous loss of SMAD4 [data not shown]. Co-IP experiments using endogenous SMAD3 expression validated binding of NFATc1 in primary, patient-derived PDAC cells.

In this study, we employed patient data, patient-derived xenografts, established human PDAC cell lines as well as murine primary cells and GEMMs of PDAC to investigate conditions of NFATc1/SMAD3 complex formation. By utilizing both co-IP with endogenous NFATc1 levels and overexpression as well as in situ PLA approaches, we identified both oncogenic KRAS activation and homozygous SMAD4 depletion as prerequisites allowing for NFATc1/SMAD3 complex assembly. Surprisingly, activation of TGF $\beta$ signaling was not a crucial trigger of nuclear transcription complex assembly, but increased the proportion of phosphorylated SMAD3 bound to NFATc1 and further promoted the protein interaction. Hence, we hypothesize that active TGF $\beta$ signal- 
ing plays a role in enhancing the transcriptional activity of nuclear NFATc1/SMAD3 complexes by encouraging DNA binding and preventing premature disassembly.

\subsection{Targeting NFATc1/Smad3/cJun complexes as a novel therapeutic approach in PDAC}

In the second part, we focused on the identification of potential target genes as well as regulated pathways and exploited therapeutic vulnerabilities that were associated with Smad4 deficiency.

\subsubsection{AP-1 proteins contribute to NFATc1/Smad3 complex formation}

To get a first hint regarding gene signatures jointly regulated by NFATc1 and Smad3, we cooperated with Dr. S. Nagarajan, Cambridge Research Institute, University of Cambridge for intersecting NFATc1 ChIPseq data derived from PDAC cells (Baumgart et al., 2014) with SMAD3 ChIPseq data from mammospheres in presence or absence of $0.1 \mathrm{nM}$ TGF $\beta$ for $3 \mathrm{~h}$ (Tufegdzic Vidakovic et al., 2015) [Figure 18]. Interestingly, in addition to jointly NFATc1/SMAD3 regulated genes, a fraction of genes was associated with an additional AP-1 binding motif (Angel et al., 1987), suggesting the existence of joint transcription regulation by NFATc1/SMAD3/AP-1 complexes. Chen et al. (2015) introduced NFATc1/AP-1 cooperativity in $61.8 \%$ of all NFATc1 targets, emphasizing the link between active epidermal growth factor receptor (EGFR) signaling and NFATc1/cJun complexes in PDAC. Among the AP-1 family, cJun is unique in its ability to positively regulate cell proliferation through the repression of tumor suppressor genes (Shaulian \& Karin, 2001), thus highlighting cJun as an interesting contributor to NFATc1/Smad3 transcription complexes in Smad4 deficiency. 


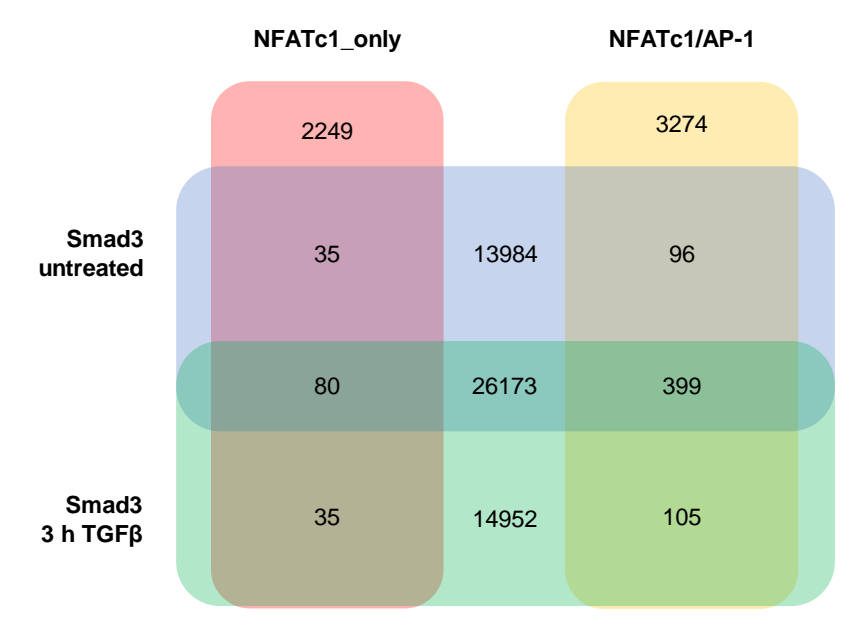

Figure 18 NFATc1 forms transcription complexes with Smad3 and AP-1 proteins. NFATc1 ChIPseq data were intersected with SMAD3 ChIPseq studies \pm TGF $\beta$. Peaks in NFATc1 ChIPseq were frequently associated with AP-1 binding motifs, resulting in subgroups of NFATc1/SMAD3 or NFATc1/SMAD3/AP-1 jointly regulated genes.

As cJun is a known Smad3 interacting protein (Zhang et al., 1998; Wong et al., 1999; Dennler et al., 2000), we focused on investigating the interplay of NFATc1, Smad3 und cJun in PDAC cells [Figure 19 A]. Via co-IP studies, we confirmed the formation of nuclear NFATc1/Smad3/cJun complexes in NKC-II Smad4 k.o. cells. Via siRNAmediated knock down of cJun and Smad3, we aimed to dissect whether interference with one of the complex partners is sufficient to disassemble the whole transcription complex. Accordingly, input samples served as knock down efficiency controls. As expected, single knock down lead to a decrease of the respective co-immunoprecipitated protein band. Interestingly, both single cJun or Smad3 knock down resulted in severe decline of the other protein, implicating that cJun silencing also interferes with Smad3 binding to HA-tagged NFATc1. The differences in cJun binding to NFATc1 subsequent to cJun knock down and Smad3 knock down, respectively, were clearly decreased with a p-value of 0.084, suggesting that cJun is able to bind NFATc1 in absence of Smad3 albeit with decreased efficiency.

Next, we aimed to check if Nfatc1 proteins are indispensable for Smad3/cJun complex formation. Therefore, we employed KPC Bl6 cells with CRISPR/Cas9-mediated 

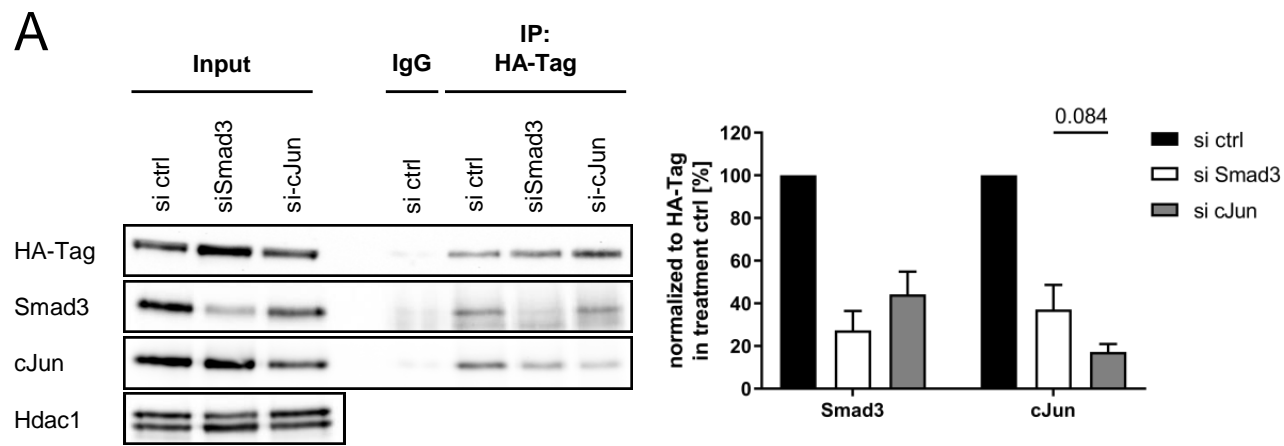

NKC-II Smad4 k.o.: murine; Kras ${ }^{\mathrm{G} 12 \mathrm{D}}$; NFATc1-HA c.n.

B

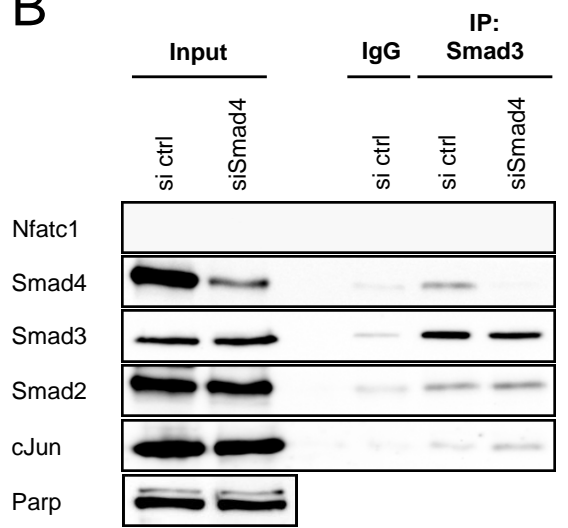

KPC BI6 NFATC1 k.o.: murine; Kras ${ }^{\mathrm{G} 12 \mathrm{D}}$; p53 $3^{\mathrm{R} 172 \mathrm{C}}$

Figure 19 cJun contributes to NFATc1/Smad3 complex formation. A Smad3 and cJun knock down studies were combined with co-IP experiments in NKC-II Smad4 k.o. cells. Western blot analysis revealed alterations in nuclear NFATc1 complex assembly caused by cJun and Smad3 knock down, respectively. Protein band intensity of co-immunoprecipitated Smad3 and cJun was measured with FIJI and normalized to respective HA-Tag signal $(\mathrm{n}=3)$. B Nuclear lysates of KPC Bl6 Nfatc1 k.o. cells \pm Smad4 knock down were conducted to co-IP experiments with subsequent western blot.

Nfatc1 k.o. for Smad4 knock down studies [Figure 19 B]. Nuclear lysates were conducted to co-IP studies utilizing endogenous Smad3 protein levels. We hereby observed enforced Smad3/cJun complex assembly subsequent to Smad4 knock down, while coimmunoprecipitated Smad2 protein was indifferent to Smad4 expression levels. In agreement with Zhang et al. (1998), our data confirm Smad3/cJun complex formation 
irrespective of Nfatc1 status, but in contrast, it provides evidence that Smad4 deficiency severely enhances cJun binding to Smad3. However, this experimental approach does not allow conclusions if Nfatc1 protein expression further supports Smad3/cJun complex formation. Together, we identified the existence of Nfatc1/Smad3/cJun complexes in PDAC and concluded that those nuclear transcription complexes are preferably assembled in Smad4-deficient PDAC cells.

Investigating circumstances promoting nuclear NFATc1/Smad3/cJun complex formation was the first step to elucidate jointly regulated gene signatures and ultimately, develop a targeted therapy approach to exploit PDAC vulnerabilities caused by Smad4 deficiency. Targeting NFATc1 is problematic as most inhibitors target upstream calcineurin signaling which plays an important role in immune response and therefore lacks a selective effect on tumor growth. The use of TGF $\beta$ inhibitors, or more specific Smad3 inhibitors, deprive us from exploiting tumor-suppressive TGF $\beta$ signaling, which is an effective tool in restraining tumor growth (Hasselluhn et al., in revision). In contrast, AP-1 proteins are downstream of the Mek/Erk signaling pathway, which is fostered by oncogenic Kras activation (Johnson et al., 1996), thus representing an interesting target in PDAC therapy.

Accordingly, we checked the efficacy of the established Mek1/2 inhibitor UO126 (Favata et al., 1998) in disrupting nuclear NFATc1/Smad3/cJun complexes [Figure 20]. Cells were maintained in normal growth medium prior to adding either $1 \mu \mathrm{M}$ UO126 or the equivalent amount of DMSO for $1 \mathrm{~h}$. The nuclear fraction was isolated and tested in co-IP approaches for NFATc1/Smad3/cJun complexes. In NKC-II Smad4 k.o. cells, UO126 treatment led to severe disassembly of Smad3 and cJun proteins from HA-tagged NFATc1 used as bait [Figure $20 \mathrm{~A}$ ]. In accordance with knock down experiments conducted in the same cells [Figure 19 A], we observed concomitant significant loss of co-immunoprecipitated Smad3 and cJun proteins, respectively. To test whether the transcription complex disrupting quality of UO126 could be reproduced in another system, we employed human PaTu8988t cells overexpressing FLAG-tagged SMAD3 
A
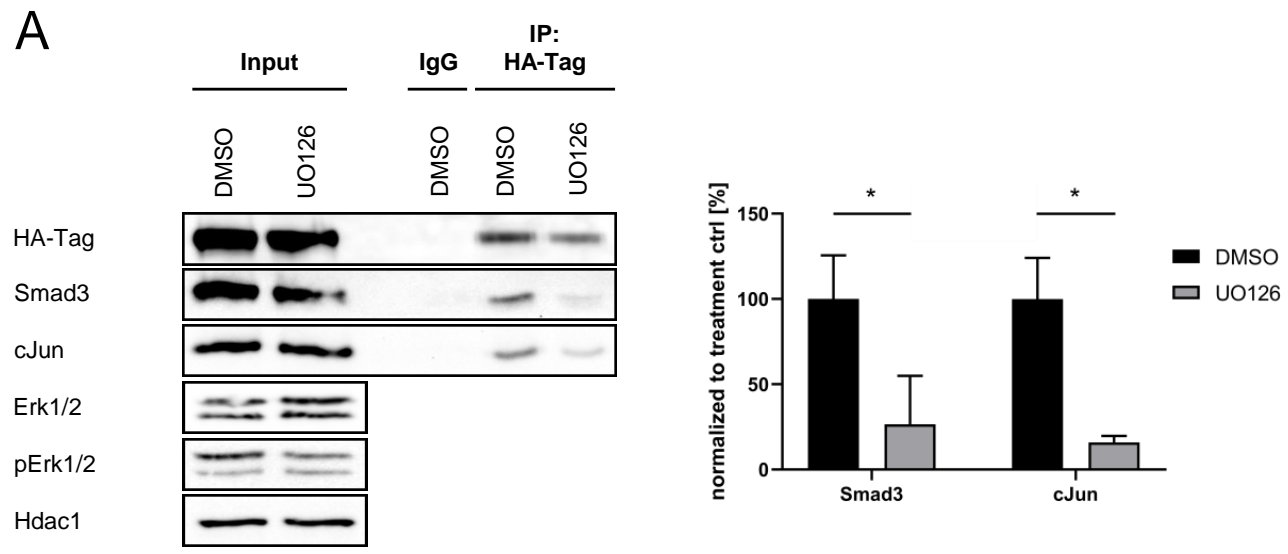

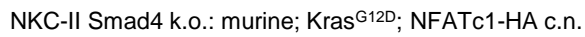

B

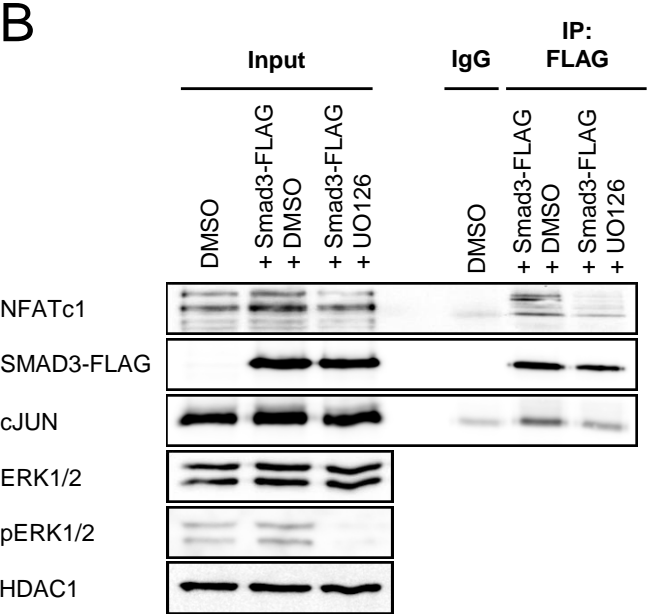

PaTu8988t: human; Kras ${ }^{\text {G12D }}$ Smad4 HD

Figure 20 Mek inhibition by UO126 disrupts nuclear NFATc1/Smad3/cJun complexes. Co-IP studies were conducted in Smad4-deficient PDAC cells treated with $1 \mu \mathrm{M}$ UO126 or DMSO for $1 \mathrm{~h}$ prior to preparation of nuclear extracts. Decrease of respective pErk1/2 signal in input samples serves as treatment control. A HA-tagged NFATc1 was utilized for detecting coimmunoprecipitated Smad3 and cJun via western blot. Protein band intensity of co-immunoprecipitated Smad3 and cJun was measured with FIJI and normalized to respective HA-Tag signal $(\mathrm{n}=3)$. B FLAG-based co-IP studies with subsequent western blotting of nuclear lysates of human PaTu8988t transfected with FLAG-tagged SMAD3. 


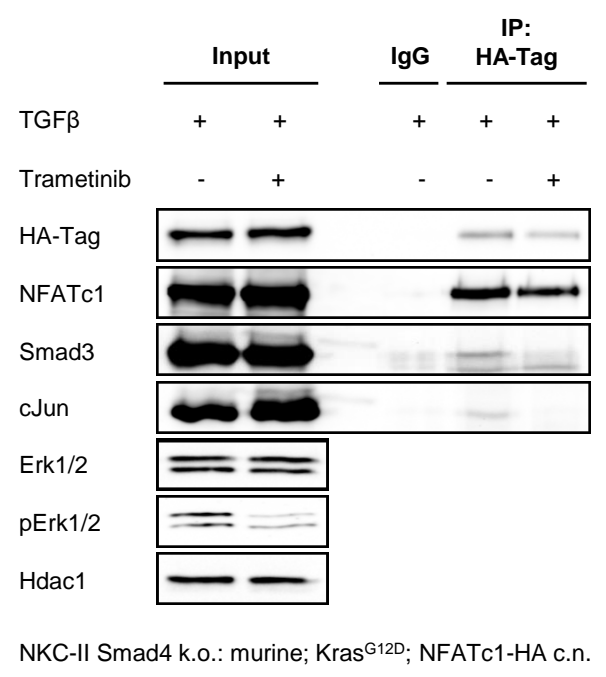

Figure 21 Mek inhibition by Trametinib disrupts nuclear NFATc1/Smad3/cJun complexes. TGF $\beta$-activated Smad4deficient NKC-II cells were treated with $5 \mathrm{nM}$ Trametinib or DMSO prior to nuclear lysate generation. Co-IP studies were conducted utilizing HA-tagged NFATc1 as bait and visualized by western blot.

followed by inhibitor treatment [Figure 20 B]. $1 \mu \mathrm{M}$ UO126 for $1 \mathrm{~h}$ effectively reduced binding of endogenous NFATc1 and cJUN proteins to SMAD3-FLAG. Together, UO126 treatment studies revealed effective reduction of NFATc1/Smad3/cJun complexes in both human and murine Smad4-deficient PDAC cells. This is in agreement with previous studies claiming that UO126 functionally antagonizes AP-1 transcriptional activity (Favata et al., 1998; Duncia et al., 1998). However, the applied dose of UO126 exceeds levels that can be employed for human PDAC patient therapy and thus was never tested in clinical trials.

Therefore, we searched for a corresponding Mek inhibitor with a lower working concentration that was already used in clinical trials and could be exploited for the disruption of NFATc1/Smad3/cJun complexes in our system. Right now, the Mek1/2 inhibitor Trametinib (Abe et al., 2011) is enrolled in 173 clinical trials, of which 8 deal with pancreatic cancer, comprising 5 designated phase 2 trials. In a first step, we aimed to reproduce NFATc1/Smad3/cJun complex disruption as a consequence of Mek1/2 inhibition by Trametinib. Therefore, we employed serum-starved and TGF $\beta$-treated 


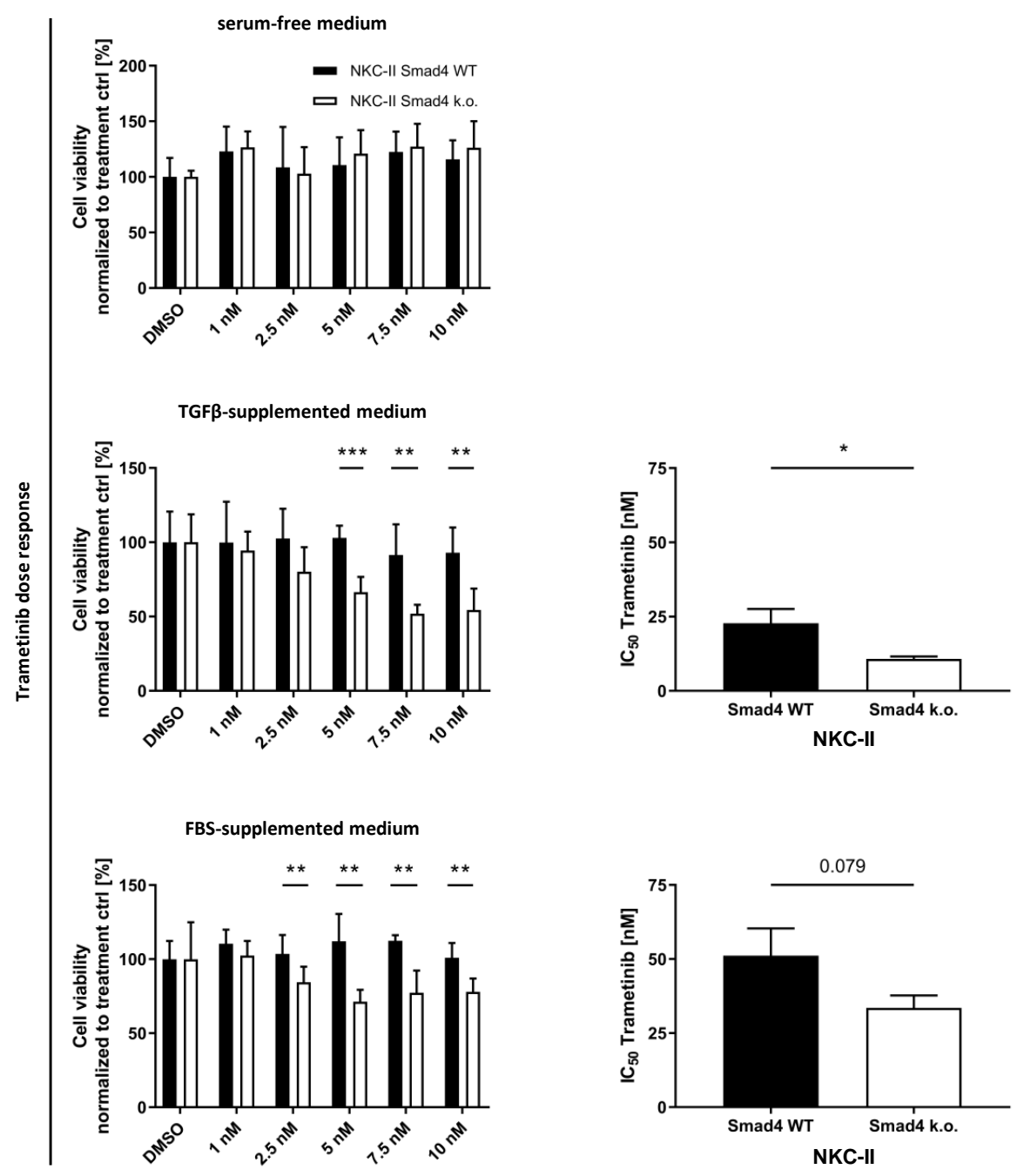

Figure 22 Smad4 deficiency promotes Trametinib sensitivity in murine PDAC cells. MTT experiments were conducted in quintuplicates in a 96 well plate with $24 \mathrm{~h}$ Trametinib treatment using indicated concentrations and DMSO as a control. Prior to treatment and incubation with indicated media, cells were serum-starved for $24 \mathrm{~h}$. $\mathrm{IC}_{50}$ was calculated from three independent experiments, respectively.

NKC-II Smad4 k.o. cells for Trametinib treatment studies with subsequent nuclear extract isolation and co-IP experiments [Figure 21]. Subsequent to Trametinib administration, decline of co-immunoprecipitated cJun and Smad3 signal was validated despite of successful HA-Tag co-IP. These data emphasized that $5 \mathrm{nM}$ Trametinib sufficiently enforced the disassembly of nuclear NFATc1/Smad3/cJun complexes in the context of active TGF $\beta$ signaling. 
In a second step, the efficacy of Trametinib was tested in increasing concentrations in MTT assays subsequent to $24 \mathrm{~h}$ serum starvation in NKC-II clones [Figure 22]. This approach comprised subsequent cultivation in different media, thereby enabling us to check for differences in Trametinib response depending on nutrients and growth factors. Trametinib treatment with continuous serum starvation did not lead to any differences in cell vitality with regards to Trametinib concentration or Smad4 status of cells, respectively. TGF $\beta$-supplemented medium promoted significant differences between NKC-II clones in Trametinib response from $5 \mathrm{nM}$ onwards. $\mathrm{IC}_{50}$ calculations revealed significant higher sensitivity of Smad4-deficient NKC-II cells towards the Mek1/2 inhibitor. Doseresponse studies in FBS-supplemented medium also promoted Trametinib efficacy in Smad4-depleted cells. Interestingly, $\mathrm{IC}_{50}$ calculations revealed the same trend as measured in TGF $\beta$-supplemented medium, but in general cells tolerated higher doses of Trametinib.

\subsubsection{Elucidation of NFATc1/Smad3/cJun regulated gene signatures in PDAC}

As the Mek1/2 inhibitor Trametinib showed to be an efficient tool to disrupt NFATc1/ Smad3/cJun complexes in Smad4 deficiency, we aimed to further scrutinize jointly regulated gene signatures of the transcription complex via RNAseq. In our setup, we included serum starvation prior to $24 \mathrm{~h}$ TGF $\beta$ and Trametinib treatment to exploit alterations in genome-wide gene expression patterns [Figure $23 \mathrm{~A}$ ]. Importantly, we also included NFATc1-depleted samples to elucidate NFATc1 as a crucial member of the transcription complex.

In parallel to RNA isolation, we harvested cotreated protein samples as treatment controls [Figure $23 \mathrm{~B}$ ]. Western blot analysis demonstrated reduced pErk1/2 levels as Trametinib treatment control and confirmed activation of TGF $\beta$ signaling by robust pSmad2/3 signal and downstream Smad4-dependent Snai1 induction in agreement with 


\section{Results}

A

B

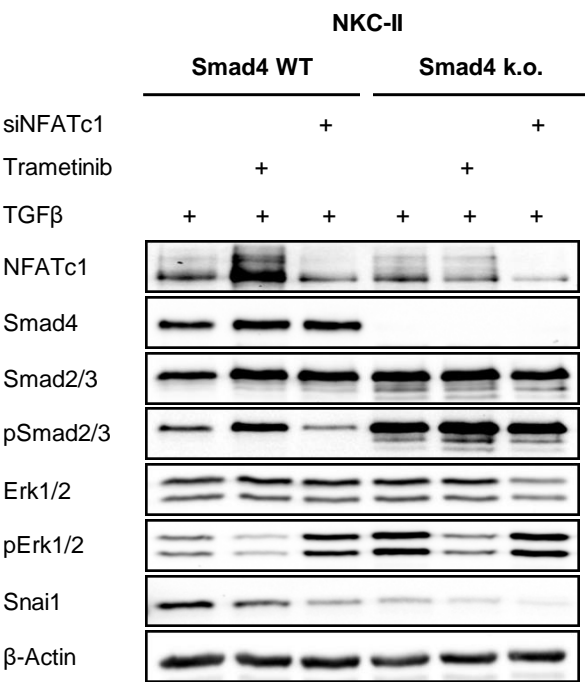

NKC-II: murine; Kras ${ }^{\mathrm{G} 12 \mathrm{D}}$; NFATc1-HA c.n.
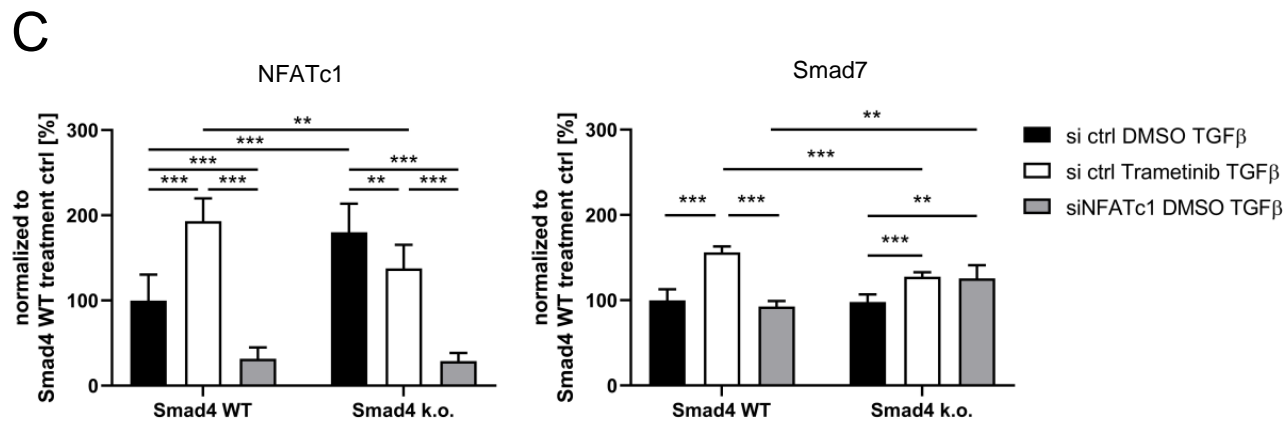

Figure 23 Smad4 status determines Trametinib response in PDAC cells. A Treatment scheme for RNAseq studies was composed of NFATc1 silencing or Trametinib treatment $(5 \mathrm{nM})$ in the context of serumstarved TGF $\beta$-treated Smad4 WT and k.o. PDAC cells. B Protein samples were harvested as treatment controls in parallel to RNA isolation. Whole cell lysates were conducted to western blot analysis to confirm Smad4 status, successful TGF $\beta$ stimulation, effective NFATc1 knock down and Trametinib treatment, respectively. C Treatment controls for NFATc1 silencing and TGF $\beta$ treatment on mRNA level were provided by qRT-PCR prior to sequencing ( $\mathrm{n}=3$, respectively).

Peinado et al. (2003). Accordingly, Smad4 status of respective cells was confirmed and NFATc1 depletion visualized. Analysis of protein pattern confirmed successful treatments and were further complemented by analyzing the corresponding RNA samples isolated in triplicates. We validated successful NFATc1 knockdown via qRT-PCR [Fig- 
ure $23 \mathrm{C}$ ]. Interestingly, Trametinib treatment led to decrease of NFATc1 expression in Smad4 deficiency, while it increased in Smad4 WT cells. As additional treatment control, we checked for Smad7 expression levels. Importantly, Smad7 expression was elevated in Trametinib-treated NKC-II Smad4 k.o. cells, suggesting abrogation of active TGF $\beta$ signaling by the inhibitory Smad.

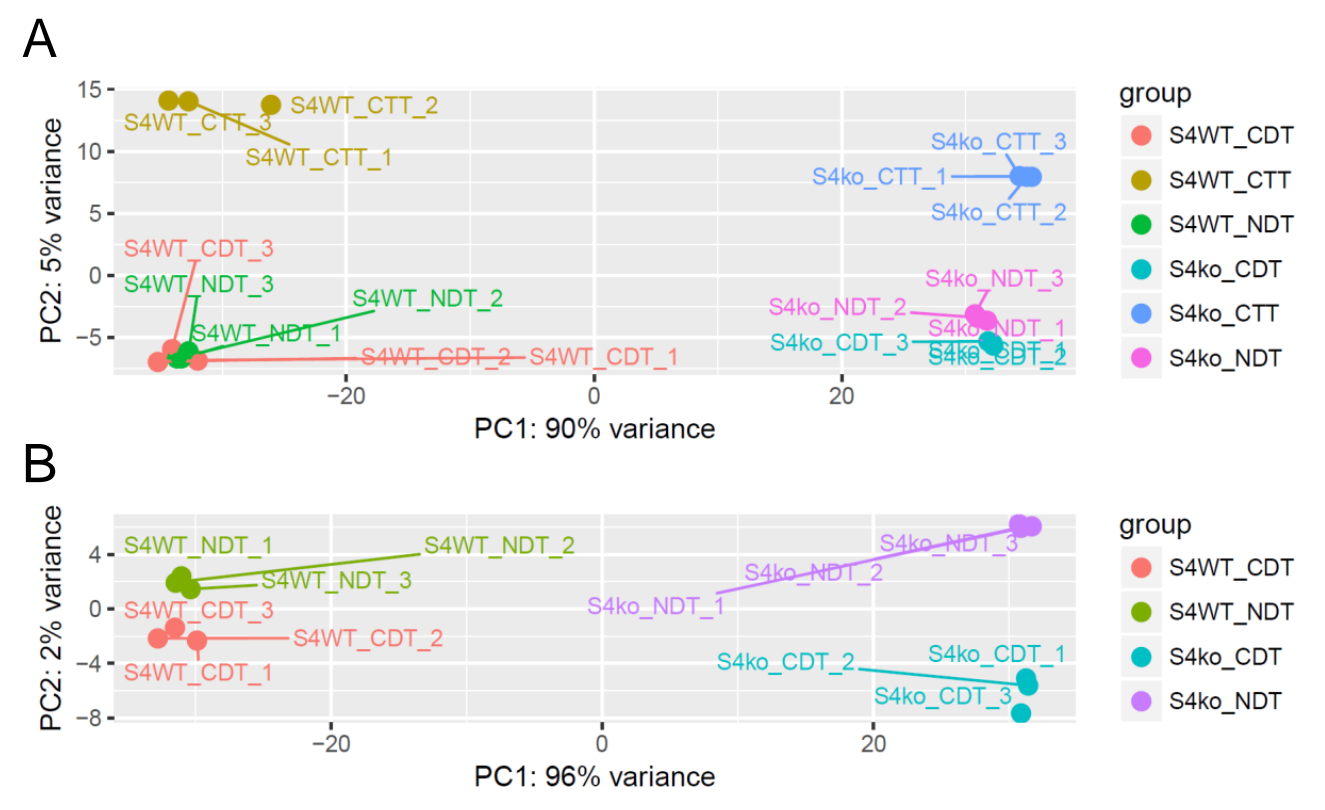

Figure 24 PCA plot of distinct RNAseq conditions. PCA plots were calculated as part of the quality control to assess similarities between replicates and differences between conditions, and Smad4 status, respectively. A PCA plot fo six distinct conditions, with Trametinib-treated samples clustering separately according to $\operatorname{Smad} 4$ status. B Detailed view on clustering of si ctrl and siNFATc1-treated samples in the context of DMSO and TGF $\beta$ reveals increased impact of NFATc1 silencing in Smad4-depleted NKC-II cells. Abbreviations: NKC-II Smad4 wildtype (S4WT), NKC-II Smad4 knock out (S4ko), si ctrl DMSO TGFß (CDT), si ctrl Trametinib TGFß (CTT), and siNFATc1 DMSO TGFß (NDT).

PCA plots were displayed to evaluate similarity among replicates and differences inbetween conditions. Clustering of all six conditions revealed major differences between Trametinib-treated samples opposed to their DMSO-treated counterparts (NKC-II si ctrl Trametinib TGF $\beta$ versus si ctrl DMSO TGF $\beta$ or siNFATc1 DMSO TGF $\beta$ ) and strong separation by Smad4 status [Figure 24 A]. Importantly, a detailed view on 


\section{Results}

\begin{tabular}{|c|c|c|}
\hline \multicolumn{3}{|c|}{ Smad4 WT si ctrl DMSO TGF $\beta$} \\
\hline $\begin{array}{l}\text { PANTHER GO } \\
\text { Slim Molecular Function }\end{array}$ & $\begin{array}{l}\text { Fold } \\
\text { Enrichment }\end{array}$ & FDR \\
\hline Integrin binding & 13.61 & $4.36 \mathrm{E}-02$ \\
\hline $\begin{array}{l}\text { Transferase activity, } \\
\text { transferring glycosyl groups }\end{array}$ & 4.83 & $1.74 \mathrm{E}-02$ \\
\hline Calcium ion binding & 4.10 & $4.15 \mathrm{E}-02$ \\
\hline Protein binding & 1.69 & $1.89 \mathrm{E}-02$ \\
\hline
\end{tabular}

\begin{tabular}{|l|l|l|}
\hline \multicolumn{3}{|c|}{ Smad4 k.o. si ctrl DMSO TGF $\beta$} \\
\hline $\begin{array}{l}\text { GO Molecular Function } \\
\text { Complete }\end{array}$ & $\begin{array}{l}\text { Fold } \\
\text { Enrichment }\end{array}$ & FDR \\
\hline $\begin{array}{l}\text { Extracellular matrix structural } \\
\text { constituent }\end{array}$ & 4.87 & $4.44 \mathrm{E}-02$ \\
\hline $\begin{array}{l}\text { Cell adhesion molecule } \\
\text { binding }\end{array}$ & 4.47 & $2.25 \mathrm{E}-03$ \\
\hline Structural molecule activity & 2.79 & $1.10 \mathrm{E}-03$ \\
\hline Cytoskeletal protein binding & 2.61 & $3.23 \mathrm{E}-04$ \\
\hline Identical protein binding & 1.80 & $4.51 \mathrm{E}-02$ \\
\hline Protein binding & 1.55 & $8.48 \mathrm{E}-12$ \\
\hline Binding & 1.35 & $2.92 \mathrm{E}-11$ \\
\hline
\end{tabular}

B

Smad4 WT si ctrl DMSO TGF $\beta$

\begin{tabular}{|l|l|l|}
\hline $\begin{array}{l}\text { PANTHER } \\
\text { Protein Class }\end{array}$ & $\begin{array}{l}\text { Fold } \\
\text { Enrichment }\end{array}$ & \\
\hline $\begin{array}{l}\text { Extracellular matrix } \\
\text { glycoprotein }\end{array}$ & 7.78 & $5.74 \mathrm{E}-04$ \\
\hline Extracellular matrix protein & 6.88 & $3.13 \mathrm{E}-06$ \\
\hline Growth factor & 6.09 & $4.53 \mathrm{E}-04$ \\
\hline Glycoyltransferase & 5.73 & $3.26 \mathrm{E}-03$ \\
\hline Protease inhibitor & 3.94 & $4.20 \mathrm{E}-04$ \\
\hline Calcium-binding protein & 3.60 & $1.56 \mathrm{E}-02$ \\
\hline Cell adhesion molecule & 3.57 & $2.49 \mathrm{E}-02$ \\
\hline Signaling molecule & 2.86 & $5.20 \mathrm{E}-04$ \\
\hline Receptor & 2.51 & $1.47 \mathrm{E}-03$ \\
\hline Enzyme modulator & 1.96 & $2.59 \mathrm{E}-02$ \\
\hline
\end{tabular}

Smad4 k.o. si ctrl DMSO TGF $\beta$

\begin{tabular}{|l|l|l|}
\hline $\begin{array}{l}\text { PANTHER } \\
\text { Protein Class }\end{array}$ & $\begin{array}{l}\text { Fold } \\
\text { Enrichment }\end{array}$ & \\
\hline Tight junction & 8.02 & $1.22 \mathrm{E}-02$ \\
\hline Cell junction protein & 4.83 & $2.06 \mathrm{E}-02$ \\
\hline Cytoskeletal protein & 2.74 & $1.16 \mathrm{E}-02$ \\
\hline
\end{tabular}

Figure 25 Smad4 deficiency alters tumor cell morphology and their interactive potential. Significantly regulated genes in NKC-II Smad4 WT si ctrl DMSO TGF $\beta$ as opposed to Smad4 k.o. (log2foldchange $\geq 1.5, \leq-1.5 ; \mathrm{q}<0.05)$ underwent GO analysis. A Comparison of positively enriched molecular functions in Smad4 WT and Smad4 k.o., respectively. B Significantly regulated genes in both treatment groups belong to different protein classes implicated in ECM modulation, cell shape, cell adhesion, and cell-cell contacts.

NFATc1 silencing (NKC-II si ctrl DMSO TGF $\beta$ versus siNFATc1 DMSO TGF $\beta$ ) demonstrated an enhanced NFATc1 signature in Smad4-deficient NKC-II cells compared to Smad4 WT [Figure 24 B]. Together, PCA plots showed strong similarity among replicates and reproduced different treatments by clustering into six distinct conditions accordingly. Prior to further analysis, a FPKM threshold $>5$ was estab- 
lished, restraining the expressed genes in our RNAseq analysis to $39.7 \%$ (9216/23 234 genes).

To correlate our RNAseq data to differences in the phenotypes of our mouse models, we identified differentially regulated genes in Smad4 WT and Smad4-deficient NKCII cells in control condition ( si ctrl DMSO TGF $\beta$; log2foldchange $\geq 1.5, \leq-1.5$; q $<0.05)$. Significantly regulated genes were further analyzed using GO tools [Figure 25]. In agreement with the phenotype observed in $S K C \mathrm{fl} / \mathrm{fl} \mathrm{p} 48$ mice as opposed to $K C$ p 48 , we demonstrated positive enrichment of molecular functions involved in cell presentation, cell contacts, and ECM adjustment. This is further supported by differences observed in positively enriched protein classes [Figure $25 \mathrm{~B}$ ], suggesting that Smad4 WT cells focus on adhesion and binding to neighboring cells while Smad4 deficiency results in alterations of the cytoskeleton and loss of cell-cell contacts. Thus, we propose that the cell identity is decisively depending on the Smad4 status of the respective PDAC cell.

Secondly, we aimed to decipher NFATc1/Smad3/cJun-driven gene signatures in Smad4depleted PDAC cells. To stratify respective gene sets, we benefitted from the thorough analysis of complex formation dependencies. As (i) Smad3/cJun complexes were formed regardless of NFATc1 expression [Figure 19 B], (ii) Smad3/cJun assembly was also observed in Smad4 WT status [Figure 19 B] and (iii) Mek inhibition was sufficient to disrupt Smad3/cJun protein binding [Figure 20 B], we needed to differentiate between NFATc1/Smad3/cJun complexes and Smad3/cJun complexes. Therefore, we focused on targets influenced by both NFATc1 silencing and Trametinib treatment in Smad4 deficiency. Consequently, we filtered for genes whose expression levels were modulated by Trametinib (NKC-II Smad4 k.o. si ctrl DMSO TGF $\beta$ versus si ctrl Trametinib TGF $\beta$; log2foldchange $\geq 0.75, \leq-0.75 ; \mathrm{q}<0.05)$ as well as NFATc1 knockdown (NKC-II Smad4 k.o. si ctrl DMSO TGF $\beta$ versus siNFATc1 DMSO TGF $\beta$; log2foldchange $\geq 0.75, \leq-0.75 ; \mathrm{q}<0.05)$. The identified genes were overlayed in a Venn diagram with Trametinib-influenced gene signatures in Smad4 WT cells (NKC-II 
A

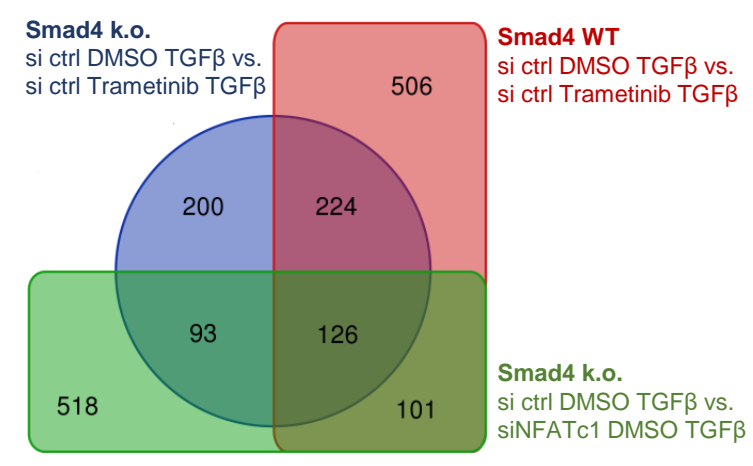

C

\begin{tabular}{|l|l|l|}
\hline Go biological process & Fold enrichment & P value \\
\hline $\begin{array}{l}\text { Pyrimidine deoxyribonucleoside } \\
\text { triphosphate metabolic process }\end{array}$ & $>100$ & $2.35 \mathrm{E}-02$ \\
\hline $\begin{array}{l}\text { Deoxyribonucleotide } \\
\text { biosynthetic process }\end{array}$ & 71.63 & $5.64 \mathrm{E}-03$ \\
\hline $\begin{array}{l}\text { Deoxyribonucleotide metabolic } \\
\text { process }\end{array}$ & 40.70 & $2.44 \mathrm{E}-03$ \\
\hline DNA replication & 14.48 & $1.61 \mathrm{E}-04$ \\
\hline Mitotic cell cycle & 5.97 & $2.27 \mathrm{E}-02$ \\
\hline $\begin{array}{l}\text { Cellular component } \\
\text { organization or biogenesis }\end{array}$ & 2.05 & $1.25 \mathrm{E}-02$ \\
\hline $\begin{array}{l}\text { Cellular component } \\
\text { organization }\end{array}$ & 2.02 & $3.78 \mathrm{E}-02$ \\
\hline
\end{tabular}

B

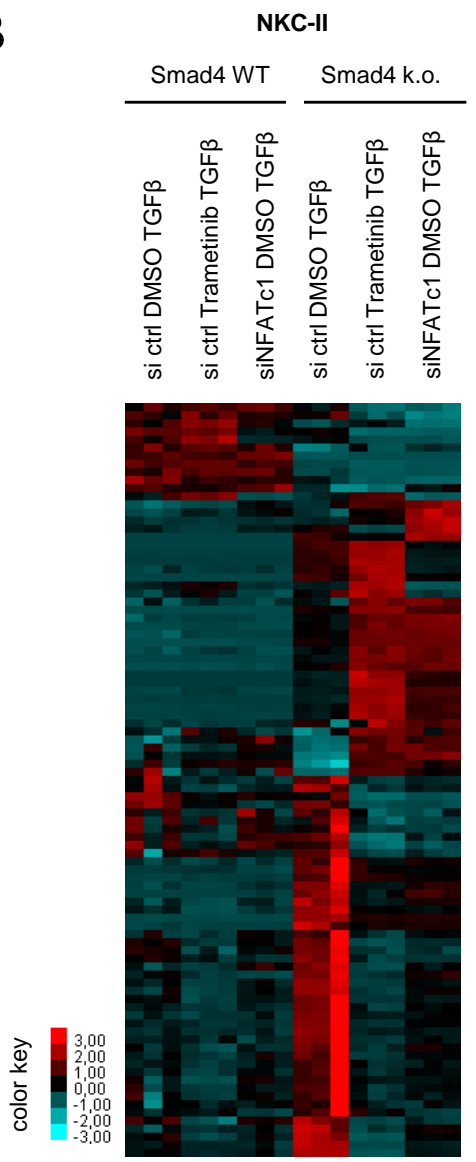

Figure 26 NFATc1/Smad3/cJun complexes interfere with proliferation, cell motility and ECM composition. A Venn diagram illustrating the identification of 93 genes accounting for the subset of potential NFATc1/Smad3/cJun targets. B Z score heatmap covering the expression levels of 93 potential complex targets and their regulation in Smad4 WT and deficiency upon Trametinib administration and NFATc1 silencing, respectively. C GO analysis of pathways enriched for potential NFATc1/Smad3/cJun targets.

Smad4 WT si ctrl DMSO TGF $\beta$ versus si ctrl Trametinib TGF $\beta$; log2foldchange $\geq$ $0.75, \leq-0.75 ; \mathrm{q}<0.05)$ to exclude false positive hits [Figure $26 \mathrm{~A}$ ]. Hence, we concluded that the 93 genes regulated by Trametinib, and NFATc1 in Smad4 deficiency, but not influenced by Trametinib in Smad4 WT situation represent the fraction of potential NFATc1/Smad3/cJun targets. The 93 potential NFATc1/Smad3/cJun targets were visualized by z score in a heatmap [Figure $26 \mathrm{~B}$ ], demonstrating on the one hand huge differences in si ctrl DMSO TGF $\beta$-treated samples depending on the Smad4 status 
and on the other hand increased Trametinib response of respective genes in Smad4 deficiency. To obtain insights into their functional relevance in Smad4-deficient PDAC, we performed GO analysis with respective target genes [Figure $26 \mathrm{C}$ ]. While most of the biological processes deal with cellular growth, there are also signatures implicated in structural changes of the cell like cell adhesion, migration, and invasion.

Next, we aimed to determine whether NFATc1/Smad3/cJun complexes could be the driving force behind the dismal prognosis of Smad4-deficient PDAC. We compared our RNAseq data to publically available datasets of unfavorable and favorable genes in PDAC (Cancer Genome Atlas Research Network et al., 2013; Uhlen et al., 2017) and demonstrated for NKC-II Smad4 k.o. cells a switch from significantly enriched dismal prognostic genes in si ctrl DMSO TGF $\beta$ to induction of favorable prognostic gene patterns upon Trametinib treatment (si ctrl Trametinib TGF $\beta$ ) [Figure 27 A]. NKC-II Smad4 WT cells in si ctrl DMSO TGF $\beta$ condition significantly correlated with unfavorable prognosis $(\mathrm{NES}=1.35, \mathrm{FDR}=0.051)$, but the Trametinib-induced switch to favorable gene expression was only confirmed in Smad4 deficiency (NKCII Smad4 WT si ctrl Trametinib TGF $\beta$ : NES $=-1.08$, FDR $=0.460$ ). In total, 415 out of 669 genes implicated in unfavorable prognosis were expressed in NKC-II cells $($ FPKM $>5)$. We performed an overlay with the 93 previously determined potential NFATc1/Smad3/cJun targets and identified 9 common genes downregulated upon Trametinib and NFATc1 knockdown in the context of TGF $\beta$, respectively. Finally, we utilized the UniProt database (UniProt Consortium, 2018) to connect potential NFATc1/Smad3/cJun targets to their respective function [Figure $27 \mathrm{~B}$ ]. Via qRT-PCR, we validated a selection of genes associated with DNA replication [Figure $27 \mathrm{C}$ ], confirming decrease of expression levels upon NFATc1 depletion, and Trametinib treatment in Smad4 deficiency. Transcription of the targets cyclin-dependent kinases regulatory subunit 1 (Cks1b), fibroblast growth factor-binding protein 1 (Fgfbp1), and minichromosome maintenance complex component 4 (Mcm4) did not decrease upon Trametinib administration in Smad4 WT cells. Importantly, none of the 93 potential NFATc1/Smad3/cJun target genes was associated with favorable prognosis in PDAC. 


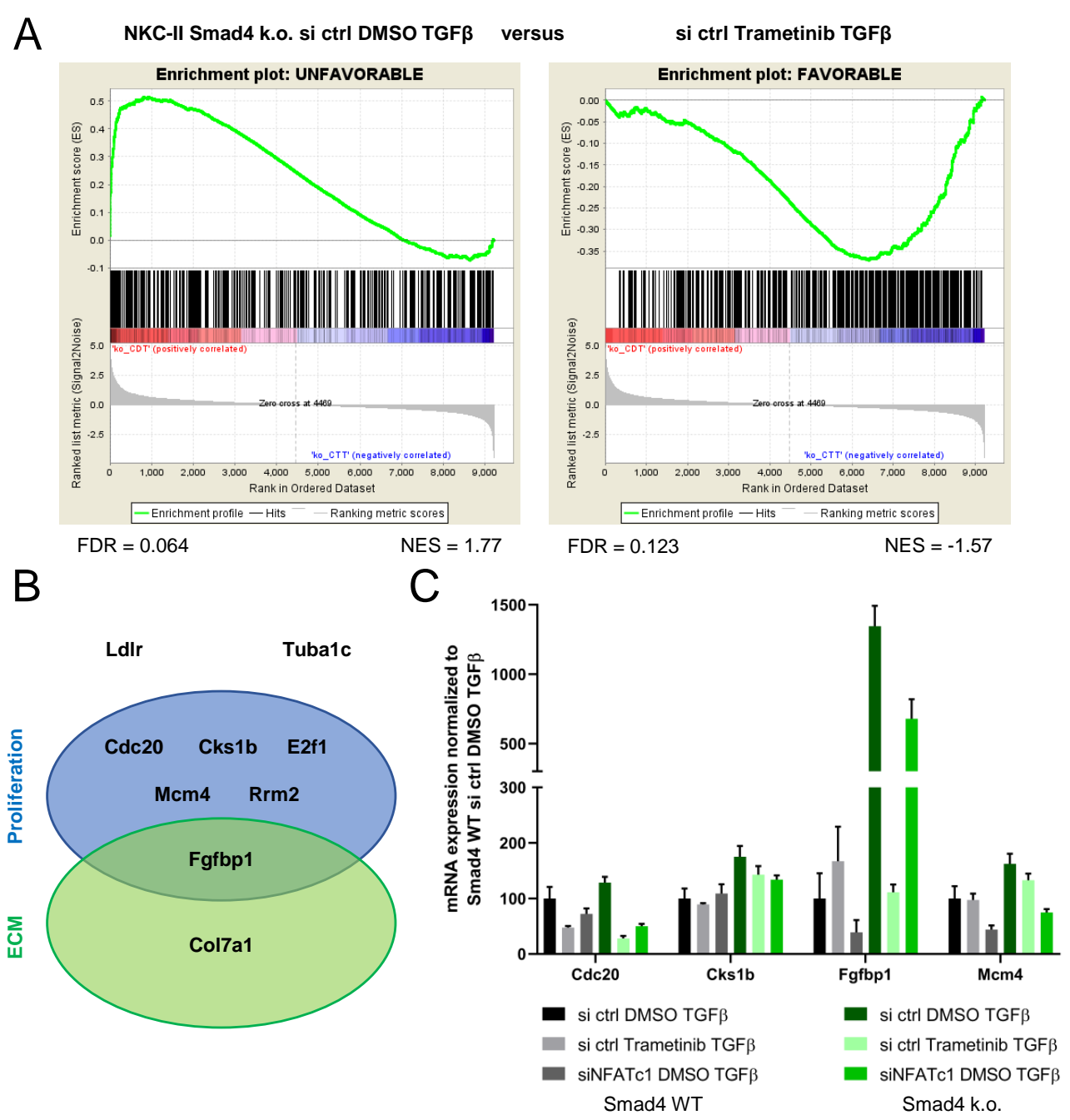

Figure 27 NFATc1/Smad3/cJun complex disruption interferes with dismal prognostic factors. A Alignment of unfavorable and favorable prognostic genes in PDAC with Smad4-deficient RNAseq data by GSEA revealed induction of favorable gene pattern upon Trametinib treatment in TGF $\beta$-induced cells. B Overlay of unfavorable prognostic genes with previously identified NFATc1/Smad3/cJun targets produced 9 jointly regulated genes. Most of them were either associated with proliferation or ECM as defined by UniProt database. C Validation of NFATc1/Smad3/cJun target selection implicated in promoting dismal outcome via qRT-PCR $(\mathrm{n}=3)$. Abbreviations: cell division cycle protein 20 homolog (Cdc20), cyclindependent kinases regulatory subunit 1 (Cks1b), collagen alpha-1 (VII) chain (Col7a1), E2f transcription factor 1 (E2f1), fibroblast growth factor-binding protein 1 (Fgfbp1), low-density lipoprotein receptor (Ldlr), minichromosome maintenance complex component 4 (Mcm4), ribonucleoside-diphosphate reductase subunit M2 (Rrm2), and tubulin alpha-1C chain (Tuba1c). 
A

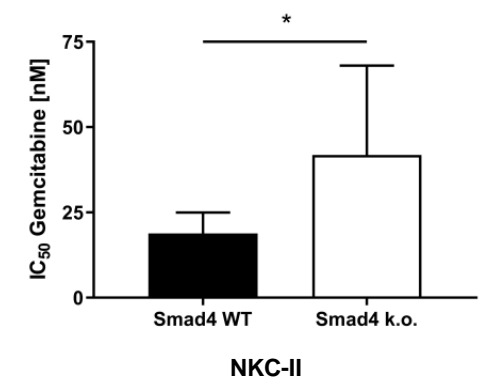

B

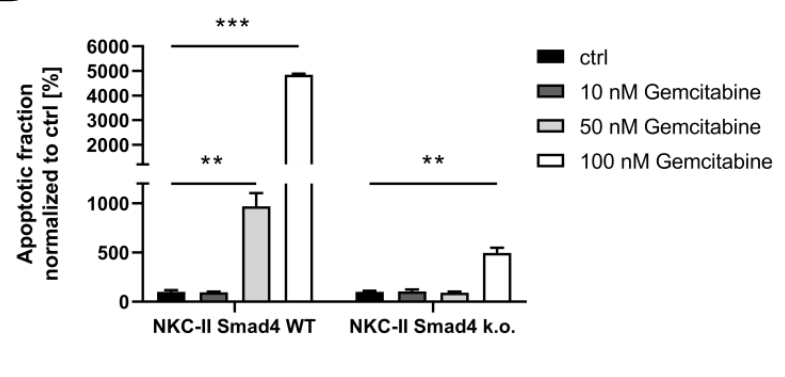

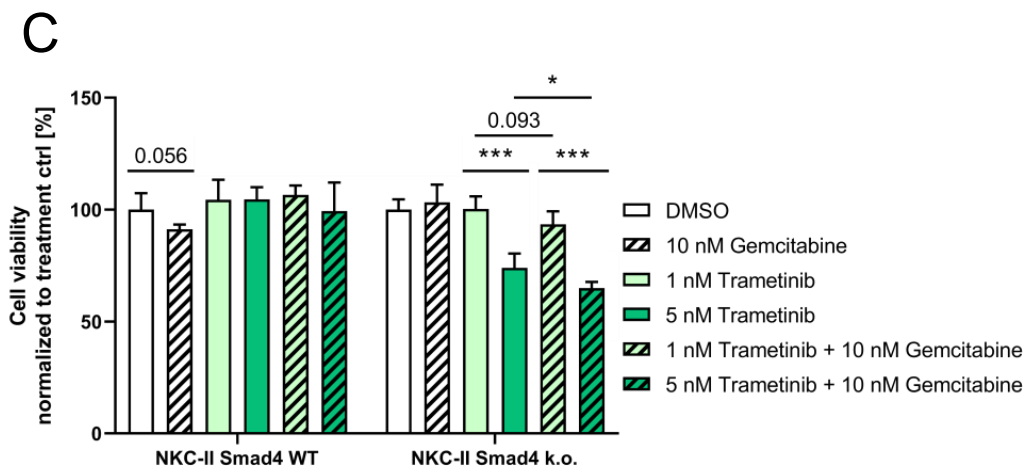

Figure 28 Smad4 deficiency enhances Gemcitabine tolerance in murine PDAC cells. A MTT experiments were conducted in quintuplicates in a 96 well plate with $72 \mathrm{~h}$ Gemcitabine treatment in increasing concentrations. $\mathrm{IC}_{50}$ was calculated from 8 independent experiments, respectively. B NKC-II clones were seeded in a 6 well plate and treated with indicated Gemcitabine concentrations for $48 \mathrm{~h}$ prior to trypsinization. Subsequent to Annexin V and propidium iodide staining, cells were conducted to flow cytometric measurement. Data from three independent experiments were analyzed by FlowJo software and the apoptotic fraction normalized to their control samples. C MTT assay in quintuplicates comprising $48 \mathrm{~h}$ Gemcitabine treatment. For the last $24 \mathrm{~h}$, Trametinib was added in indicated concentrations.

The top two hits of biological processes driven by potential NFATc1/Smad3/cJun targets were implicated in nucleoside and nucleotide metabolism [Figure $26 \mathrm{C}$ ]. As the standard of care for non-resectable, metastatic pancreatic cancer still relies on the nucleoside analog Gemcitabine in most cases (Ellenrieder et al., 2016), Smad4-dependent alterations of nucleoside metabolism are of particular interest. Subsequent studies using different CRISPR/Cas9 clones revealed that Smad4 deficiency was universally associated with increased tolerance against the tested nucleoside analogs Gemcitabine 


\section{Results}

and AraC [Figure 28 A, Appendix Figure 33]. Accordingly, Annexin V/propidium iodide staining with subsequent flow cytometry demonstrated that $48 \mathrm{~h}$ Gemcitabine treatment result in lower apoptosis rates in Smad4 deficiency compared to Smad4 WTexpressing NKC-II cells [Figure 28 B]. Vice versa, we tested if induced Gemcitabine resistance by long-term exposition to increasing Gemcitabine concentrations is mediated by Smad4 silencing [Appendix Figure 34]. We could not detect alterations is Smad4 protein or mRNA expression [Appendix Figure $34 \mathrm{~A}+\mathrm{B}$ ], thus concluding that induced Gemcitabine tolerance is either realized via regulation downstream of Smad4 or via completely different mechanisms.

Combined treatment approaches aimed to elucidate if Trametinib administration sensitized Smad4-deficient cells for Gemcitabine treatment. Indeed, we demonstrate improved chemoresponse or resensitization of NKC-II Smad4 k.o. cells upon treatment with Gemcitabine in levels below $\mathrm{IC}_{50}$ followed by Trametinib treatment in sub- $\mathrm{IC}_{50}$ concentrations [Figure $28 \mathrm{C}$ ]. Note that combined Gemcitabine and Trametinib treatment were conducted in normal cultivation medium preventing disturbances in cell proliferation. Importantly, sole Trametinib administration and combined treatment was ineffective in NKC-II Smad4 WT cells. Thus, via dose-response studies of Trametinib and Gemcitabine in NKC-II clones, we could validate increased therapeutic response to combined Gemcitabine and Trametinib treatment caused by Smad4 depletion.

In order to elucidate how loss of Smad4 caused increased tolerance to nucleoside analogs, we employed our RNAseq data to decipher altered gene signatures in NKC-II Smad4 k.o. cells that conveyed increased Gemcitabine tolerance compared to Smad4 WT cells. As we observed increased Gemcitabine susceptibility in Smad4-deficient cells subsequent to Trametinib treatment [Figure $28 \mathrm{C}$ ], we filtered for significantly altered genes (log2foldchange $\geq 1.5, \leq-1.5 ; \mathrm{FPKM}>5$ ) in NKC-II Smad4 k.o. si ctrl DMSO TGF $\beta$ versus si ctrl Trametinib TGF $\beta$ treated samples and displayed the regulated genes as z score means throughout all conditions via heatmap [Figure $29 \mathrm{~A}$ ]. Intriguingly, although we observed immense differences among si ctrl DMSO TGF $\beta$ 
A

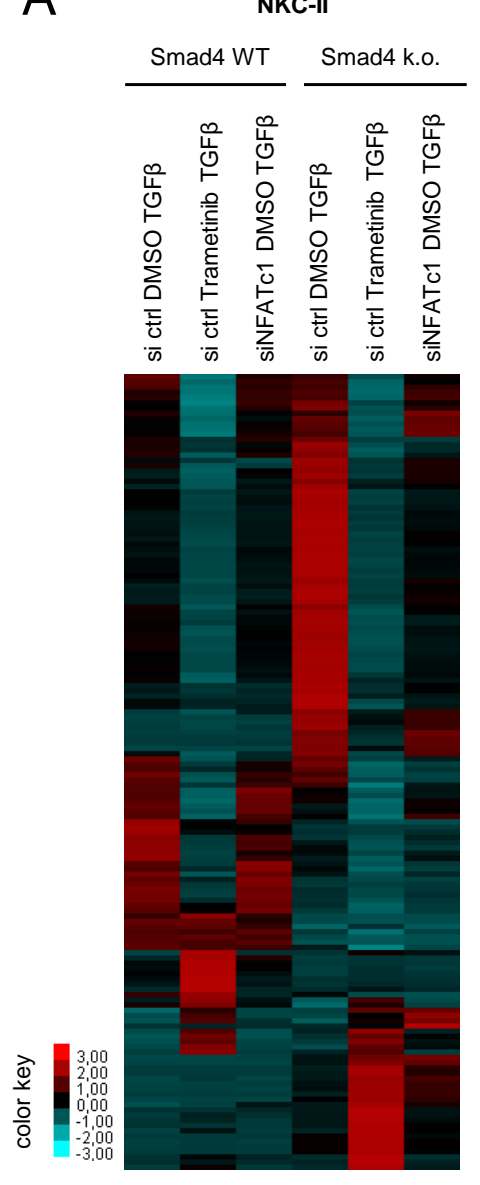

B

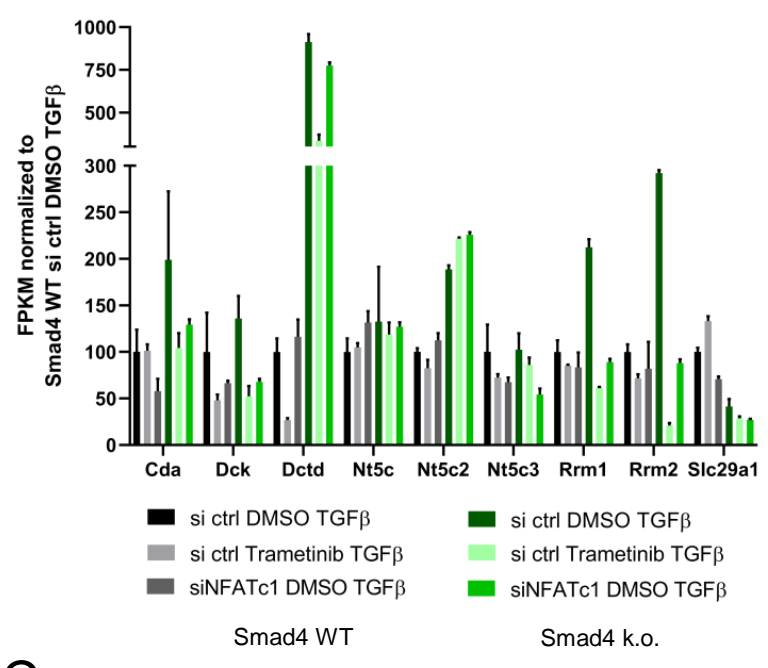

C

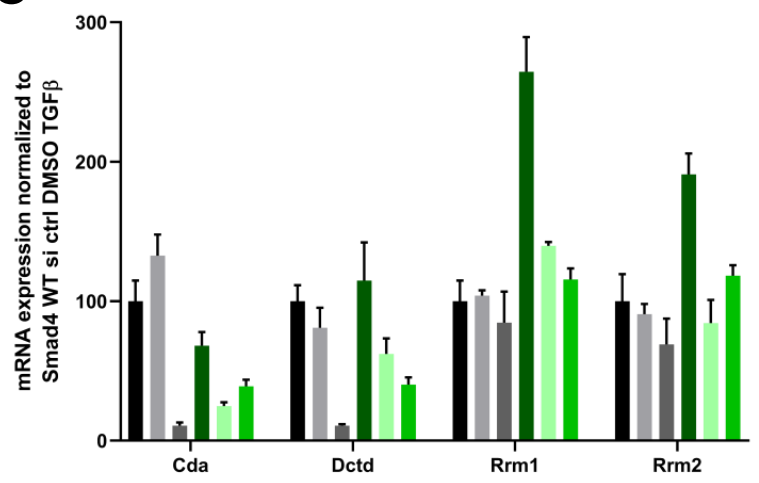

Figure 29 Trametinib administration restores Gemcitabine susceptibility. A Mean of replicates displayed as z score heatmap filtered for significant alterations between NKC-II Smad4 k.o. DMSO and Trametinib-treated conditions (log2foldchange $\geq 1.5, \leq-1.5 ; \mathrm{FPKM}>$ 5). B FPKM count normalized to NKC-II Smad4 WT si ctrl DMSO TGF $\beta$ for genes implicated in Gemcitabine metabolism $(n=3$, respectively). C Gene expression levels for Gemcitabine metabolizing enzymes evaluated by qRT-PCR $(\mathrm{n}=3)$. Abbreviations: cytidine deaminase (Cda), deoxycytidine kinase (Dck), deoxycytidylate deaminase (Dctd), 5'(3')-deoxyribonucleotidase, cytosolic type (Nt5c), cytosolic purine 5'-nucleotidase (Nt5c2), cytosolic 5'-nucleotidase $3 \mathrm{~A}$ (Nt5c3), ribonucleoside-diphosphate reductase large subunit (Rrm1), and equilibrative nucleoside transporter 1 (Slc29a1). 
samples depending on Smad4 status, Trametinib administration led to an astonishing approximation of the gene regulation pattern in Smad4 WT and k.o. condition (si ctrl Trametinib TGF $\beta$ ). This harmonization was in line with increased Gemcitabine response detected after Trametinib treatment in Smad4-deficient cells. In addition, we compared our RNAseq data to a gene list comprising important mediators of Gemcitabine and AraC metabolism provided by Li et al. (2008). 9 out of 17 genes met the FPKM threshold (52.9\%) and their FPKM expression levels normalized to NKC-II Smad4 WT si ctrl DMSO TGF $\beta$ were displayed [Figure 29 B]. Smad4 deficiency led to enhanced expression of cytidine deaminase (Cda), deoxycytidylate deaminase (Dctd), cytosolic purine 5'-nucleotidase (Nt5c2), ribonucleoside-diphosphate reductase large subunit (Rrm1), and ribonucleoside-diphosphate reductase subunit M2 (Rrm2) transcripts, respectively. Of note, Slc29a1 encodes for a transporter enabling cellular uptake of Gemcitabine, representing the only nucleoside transporter verifiably transcribed in our PDAC cells. All other genes are directly involved in Gemcitabine metabolism, except for Rrm1 and Rrm2, respectively.

Based on these results, we identified four promising candidates for increased Gemcitabine tolerance in Smad4 deficiency that could be abrogated by Trametinib treatment and validated their regulation in qRT-PCR experiments [Figure $29 \mathrm{C}$ ]. The enzymes Cda and Dctd directly interfere with Gemcitabine metabolism, enforcing premature degradation with subsequent transfer out of the cell, while Rrm1 and Rrm2 together catalyze the biosynthesis of deoxyribonucleotides. The latter compete with phosphorylated Gemcitabine in DNA strand incorporation (Tanaka et al., 2010). Thus, we identified candidate genes whose upregulation in Smad4-deficient PDAC cells might convey increased Gemcitabine tolerance which could be counteracted by supplementary Trametinib treatment. Importantly, Rrm2 was previously identified as one of the potential NFATc1/Smad3/cJun targets implicated in dismal prognosis, suggesting that oncogenic NFATc1/Smad3/cJun-driven signatures contributed to increased Gemcitabine tolerance in Smad4-deficient PDAC cells. 
Our RNAseq approach revealed signatures of potential NFATc1/Smad3/cJun targets being involved in triggering an unfavorable prognosis in PDAC patients, alteration of the tumor microenvironment, adaptation of cell morphology towards a more aggressive and invasive phenotype, and impairment of nucleoside analog-based cytotoxicity. These features corresponded with the hallmarks of Smad4-deficient PDAC identified in our mouse model. Importantly, disruption of nuclear NFATc1/Smad3/cJun complexes by Mek inhibitor Trametinib restored Gemcitabine response and altered the transcriptional landscape profoundly towards favorable gene signatures for improved survival of PDAC patients. 



\section{Discussion}

\subsection{Smad4 deficiency in pancreatic cancer favors invasiveness and aggressiveness of disease}

Since the discovery of the tumor suppressor gene Smad4 by Hahn et al. (1996b), its pivotal role in pancreatic cancer exceeds against other cancer entities. Loss of Smad4 occurs in half of all invasive PDAC patients (Wilentz et al., 2000a) and is associated with dismal prognosis, thus defining the subgroup of Smad4-depleted PDAC as an interesting target in precision medicine. Elucidating the mechanisms behind aggressive, tumor-promoting gene signatures promoted by loss of Smad4 is an important step in understanding cancer progression and thus development of therapeutic approaches exploiting cancer vulnerabilities. Patient stratification is a first step in the direction of personalized medicine, allowing to screen a patient cohort for specific genetic markers to ensure tailored treatment regimes.

Loss of Smad4 in PDAC is associated with decreased patient survival and increased metastatic spread (Shin et al., 2017), characterizing the Smad4 status as a prognostic factor in human PDAC. The common Smad has a pivotal role in the TGF $\beta$ pathway and its depletion is discussed to mediate the switch from tumor-suppressive to oncogenic TGF $\beta$ signaling (Zhang et al., 1997). However, the exact mechanism on how the TGF $\beta$ cascade is exploited for tumor promotion remains elusive. We hypothesized that receptor Smads form alternative transcription factor complexes in absence of Smad4, regulating differential gene signatures. In this study, we first identified nuclear NFATc1/Smad3/cJun transcription factor complexes in PDAC and scrutinized conditions facilitating or abrogating this complex formation. We demonstrated that Smad4 deficiency is crucial to allow NFATc1/Smad3/cJun complex assembly in various human and murine PDAC cell lines. This was supplemented by murine in vivo PLA studies, confirming the rise of Nfatc1/Smad3 signal upon homozygous loss of Smad4. 
Moreover, we discovered that the transcription complex is depending on oncogenic Kras activation, thus limiting its occurrence to Kras-mutated PDAC as opposed to healthy pancreatic tissue and scarce Kras-independent PDAC. In this work, we aimed to comprehend features of Smad4-deficient PDAC by studying pancreatic carcinogenesis and metastasis in an in vivo model accompanied by functional in vitro assays utilizing CRISPR/Cas9-mediated genome editing to establish Smad4 WT and k.o. clones with similar genetic background.

\subsubsection{Smad4 depletion cooperates with oncogenic Kras activation in strong acceleration of PDAC formation}

The SKC fl/fl p48 mouse model faithfully recapitulated severe reduction of life expectancy in comparison to Smad4 WT-expressing KC p48 mice as already observed for human PDAC patients from TCGA database. Increased aggressiveness of disease was reproduced by enforced initiation of preneoplastic lesion formation and early tumorigenesis. The enhanced metastatic burden observed in SKC $\mathrm{fl} / \mathrm{fl} \mathrm{p} 48$ mice is in agreement with patient autopsy data provided by Iacobuzio-Donahue et al. (2009) and resected human PDAC biopsies by Shin et al. (2017). There was no significant correlation between Smad4 status and PDAC stage for the patient cohort of TCGA [data not shown], while homozygous Smad4 depletion resulted in exclusively low PDAC stage (G1) in our GEMM which is supported by previous data of Vincent et al. (2012). This is in contradiction to proposed acceleration of PDAC invasiveness which suggested increased dedifferentiation featured by loss of Smad4. We observed excessive gain of pancreas weight in a short period of time covering the age of approximately 6 to 12 weeks. At the same time, respective $S K C$ fl/fl p48 mice suffered from a drastic loss of body weight. We suggest that premature death due to extreme tumor burden impaired the acquisition of additional mutations or epigenetic alterations to trigger tumor cell dedifferentiation. 
In agreement with Izeradjene et al. (2007), we utilized the p48-Cre recombinase to target both $\operatorname{Kras}^{G 12 D}$ as well as heterozogous or homozygous Smad4 depletion to the pancreas. The Pdx1-Cre recombinase used by Kojima et al. (2007) in the context of Smad deficiency can lead to severe extrapancreatic effects, while p48-Cre is more tightly confined to the pancreas (Offield et al., 1996; Hingorani et al., 2005; Izeradjene et al., 2007; Gades et al., 2008). In contrast to Izeradjene et al. (2007), we observed significant decrease of lifespan of mice bearing Smad4 haploinsufficiency $(S K C \mathrm{fl} /+\mathrm{p} 48)$ compared to Smad4 WT (KC p48). We employed 20 individuals of each genotype for our survival studies while insignificant results of Izeradjene et al. (2007) might be explained by the small number of $K C p 48$ animals $(K C p 48: \mathrm{n}=6$; SKC $\mathrm{fl} /+\mathrm{p} 48$ : $\mathrm{n}=22$ ). In addition, homozygous Smad4 depletion in the context of oncogenic Kras activation ( $\mathrm{SKC}$ fl/fl p48) reduced survival to 4.2 months in our study as opposed to 8 months $(\mathrm{n}=18)$ disclosed by Izeradjene et al. (2007). Importantly, our SKC $\mathrm{fl} / \mathrm{p} 48$ and SKC fl/fl p48 models rely on the same GEMMs (Yang et al., 2002; Hingorani et al., 2003), proposing that our end-point criteria were more strictly defined as in the previous study by Izeradjene et al. (2007).

The second hit theory by Yachida \& Iacobuzio-Donahue (2013) implicated that in human PDAC, genetic inactivation of TP53 in advanced PanIN stage is a prerequisite for SMAD4 deficiency in established PDAC. As our mouse model was based on Tp53 WT, the decisive genetic background for dedifferentiated PDAC might have been neglected. Studies by Whittle et al. (2015) combining oncogenic Kras activation and homozygous Smad4 depletion with Tp $53^{R 172 H /+}$ under pancreas-specific p48-Cre demonstrated no additional decrease in mouse lifespan (125 days median survival versus 129 days in our SKC fl/fl p48 mice). However, Whittle et al. (2015) described the occurrence of dedifferentiated areas in their GEMM, thereby suggesting that our SKC fl/fl p48 model retained well-differentiated PDAC based on Tp53 WT status.

In addition, Singh et al. (2015) demonstrated that Tp53 mutation in the $\operatorname{Kras}^{G 12 D}$; $p 53^{R 172 C /+}$; pdx-Cre (KPC) model and human PDAC is insufficient in driving ded- 
ifferentiation and is not correlated to higher tumor grading. Thus, they introduced NFATc1 as a major driver of dedifferentiation in the context of Tp53 mutation. In our GEMMs, we did not systematically assess NFATc1 abundance via IHC. Instead, we determined NFATc1 expression levels in primary PDAC cell lines isolated from tumorbearing mice of respective genotypes, revealing only weak NFATc1 signal [data not shown]. We conclude that highly differentiated PDAC observed in SKC $\mathrm{fl} / \mathrm{fl} \mathrm{p} 48$ mice relies at least partly on low NFATc1 activity. This is in agreement with Diaferia et al. (2016) describing strong NFATc1 signal as a hallmark of high-grade, undifferentiated PDAC cells. On the molecular level, NFATc1-dependent transcription introduces inflammatory and proliferative signatures in PDAC (Buchholz et al., 2006). Importantly, NFATc1 preferably cooperates with additional transcription factors for gene regulation (Loh et al., 1996; Chen et al., 1998; Baumgart et al., 2014). Although Smad3/cJun complexes are already described by Zhang et al. (1998), the participation of the inflammatory transcription factor NFATc1 as well as the promoted assembly upon Smad4 deficiency identified in our study add new aspects to the proposed transcription complex. As the function of cJun is highly context-dependent (Eferl \& Wagner, 2003), the introduction of an inflammatory tumor environment by NFATc1 and activated TGF $\beta$ signaling (Ben-Neriah \& Karin, 2011; Baumgart et al., 2014) adjust the broad spectrum of cJun-regulated genes to their full oncogenic potential. Consequently, triggering NFATc1 expression by an inflammatory insult like caerulein could result in more dedifferentiated PDAC formation.

\subsubsection{Advantages of CRISPR/Cas9-mediated Smad4 knock out in PDAC research}

In this work, investigating functional implications of Smad4 deficiency in pancreatic cancer in vitro relied on comparison of Smad4 WT and k.o. clones generated by CRISPR/Cas9-mediated genome editing. Although there has been a lot of research identifying Smad4-dependent TGF $\beta$ functions in PDAC (Schwarte-Waldhoff et al., 
2000; Duda et al., 2003; Subramanian et al., 2004; Jonckheere et al., 2004; Yasutome et al., 2005; Jazag et al., 2005; Bera et al., 2013; Leung et al., 2013; Fullerton et al., 2015) this new technique offers a novel approach to elucidate contradictory data published by other groups. In the past, gene silencing of Smad4 was exploited to scrutinize Smad4-dependent TGF $\beta$ signaling and functions (Jazag et al., 2005; Bera et al., 2013; Leung et al., 2013). In contrast to the status observed in patient tissue, where Smad4 is either present or completely absent in epithelial PDAC cells, knock down experiments maintain residual Smad4 expression which can lead to undesired cross reactions or prevent the establishment of gene signatures relying on total Smad4 deficiency. Accordingly, we did not employ Smad4 knock down experiments in this work if avoidable, allowing us to remain closer to the human PDAC situation. Secondly, established human SMAD4-deficient PDAC cell lines were exploited in the context of SMAD4 overexpression studies to recapitulate alterations in TGF $\beta$ response due to SMAD4 status (Schwarte-Waldhoff et al., 2000; Duda et al., 2003; Subramanian et al., 2004; Jonckheere et al., 2004; Yasutome et al., 2005; Fullerton et al., 2015), leading to considerable discrepancies in conclusions. Long-term adaptations to SMAD4 depletion might lead to accumulation of additional mutations and alterations of chromatin landscape, resulting in inconsistencies observed in the aforementioned literature. Therefore, reintroduction of SMAD4 constructs might not lead to reestablishment of conventional TGF $\beta$ signaling. In our experimental setup, Smad4 overexpression studies were only used for investigating conditions favoring transcription complex assembly. Here, we focused on Smad4 protein expression traced by western blot, regardless of the upstream signaling pathways. Thirdly, established human PDAC cell lines with known SMAD4 status were compared among each other (Duda et al., 2003; Subramanian et al., 2004). Thereby, huge variations of the genetic background of each cell line are neglected which potentially shape Smad4-dependent signatures. As pancreatic cancer is characterized by its extreme genetic heterogeneity, this procedure elucidated robust SMAD4-dependent pathways and functions but might also lead to false-positive findings were SMAD4 deficiency might be cooperating with TP53 and KRAS mutations 
in driving certain gene signatures. Importantly, in these models, functional relevance of SMAD4 in active TGF $\beta$ signaling might be missed due to data inconsistency.

The CRISPR/Cas9-mediated Smad4 depletion offers the unique chance to compare Smad4-dependent functions and gene signatures in cells harboring the same genetic background. We established CRISPR/Cas9-derived Smad4 k.o. clones and their respective Smad4 WT counterparts in two murine and one human PDAC cell line, thereby covering different genetic backgrounds. Importantly, all cellular systems relied on oncogenic Kras activation as a major driver of pancreatic carcinogenesis. We identified nuclear NFATc1/Smad3/cJun complexes exclusively in Smad4-deficient PDAC, thereby confirming our hypothesis of alternative Smad3 binding partners in Smad4 deficiency. Smad3 and Smad4 interact via their conserved carboxy-terminal mad homology (MH2) domains which are also used for further association with DNA binding proteins and transcriptional activators (Zhang et al., 1997; Attisano \& Wrana, 2000). The aminoterminal MH1 domain autoinhibits the MH2 domain, mediates DNA binding and also promotes higher order complex assembly with additional DNA-binding partners (Attisano \& Wrana, 2000). We hypothesized that NFATc1/Smad3 interaction is mediated via MH2 domain, offering a potential explanation of NFATc1/Smad3 complex assembly exclusively in Smad4-deficient PDAC and suggesting comparably stronger binding affinity of Smad4 and Smad3 proteins. However, this hypothesis could be tested in future experiments by introducing a Smad4 construct only covering the MH2 domain in Smad4-depleted PDAC cells and investigation of potential NFATc1/Smad3 complex disruption.

In this work, we only commented on the influence of NFATc1 in shaping Smad3dependent transcription without ruling out the possibility of other transcription factors hijacking Smad3 as a complex partner in the context of Smad4 depletion. However, without claiming to disclose all potential Smad3 binding partners in Smad4 depleted PDAC, we cannot draw any conclusions on the importance of NFATc1 in relation to other alternative binding partners for Smad3. Thus, we focus on NFATc1/Smad3/cJun- 
mediated transcription promoting oncogenic gene signatures in Smad4 deficiency without assigning every phenotypical feature observed upon loss of Smad4 to this transcription complex.

To determine conditions beneficial for nuclear NFATc1/Smad3/cJun complex assembly, the majority of experiments conducted in this work relied on murine NKC-II Smad4 WT and k.o. clones. Conditional nuclear HA-tagged NFATc1 facilitated co-IP studies and, in agreement with Baumgart et al. (2014) and Singh et al. (2015), led to an aggressive phenotype recapitulating invasive PDAC in vitro. Consequently, RNAseq analyses in TGF $\beta$-treated NKC-II clones revealed significant induction of unfavorable prognostic gene signatures in PDAC irrespective of Smad4 status (Cancer Genome Atlas Research Network et al., 2013; Uhlen et al., 2017). However, we identified a potential subset of genes related to dismal prognosis differentially induced by NFATc1/Smad3/cJun complexes in Smad4 deficiency. Those genes were not only implicated in driving proliferation but also ECM modulation, synergizing in driving tumor growth. The restrictions used to narrow down signatures of NFATc1/Smad3/cJun complexes in Smad4 deficiency relied on the conditions identified by co-IP approaches, thus ensuring involvement of the three transcription factors in the regulation of identified genes. However, this RNAseq approach did not allow any spatial or temporal resolution on transcription factor binding to DNA. Future ChIP experiments could contribute to the definition of DNA regions were NFATc1, Smad3, and cJun bind and regulate their target gene expression in a transcription complex.

In this study, we failed to define the importance of activated TGF $\beta$ signaling for NFATc1/Smad3/cJun transcription complex formation. We detected an increase in co-immunoprecipitated Smad3 to NFATc1 subsequent to TGF $\beta$ administration which was accompanied by increasing shares of Smad3 phorsphorylation at the carboxyl terminal serine residues mediated by TßR-I (Zhang et al., 1996). However, Smad3 is located predominantly in the nucleus (Chen et al., 1996; Zhang et al., 1997; Yasutome et al., 2005) independent of its phosphorylation status. We proposed that activated 
TGF $\beta$ signaling fostered Smad3-dependent transcription and thus the transcriptional output of the whole NFATc1/Smad3/cJun complex. Importantly, the relevance of diverse Smad3 phosphorylation sites in shaping its target gene regulation (Kretzschmar et al., 1999; Sekimoto et al., 2007) while not affecting DNA binding affinity (Zawel et al., 1998) has already been described. Future investigations dealing with Smad4-dependent alterations of Smad3 phosphorylation occupancy could elucidate the molecular mechanisms behind gene signature variations as a consequence of $\operatorname{Smad} 4$ deficiency.

Assessing functional implications of Smad4 depletion using CRISPR/Cas9 clones in vitro aimed to comprehend features of Smad4-deficient PDAC observed using GEMMs in vivo. In a first attempt, we aimed to elucidate data discrepancies (Duda et al., 2003; Subramanian et al., 2004; Jazag et al., 2005) regarding the role of Smad4 in TGF $\beta$-mediated migration. Thereby, we ensured that TGF $\beta$ response upstream of Smad4 was unaffected in our CRISPR/Cas9 clones. We confirmed TGF $\beta$-mediated increase in migration speed in both $\operatorname{Smad} 4 \mathrm{WT}$ and k.o. cells and could demonstrate at the same time that the migrative capacity of Smad4-deficient cells exceeded their respective counterpart. Cell migration is a complex process based on iterated detaching and reattaching steps combined with alterations of cell polarity and cytoskeletal rearrangement (Dreymueller et al., 2017). Our RNAseq data suggested involvement of NFATc1/Smad3/cJun complexes in driving genes associated with cytoskeletal organization, thus providing a potential link how increased migrative potential was promoted in Smad4 deficiency. However, effective migration potential is one crucial prerequisite of metastasis formation, as PDAC cells have to leave their original cell compound and enter vessels to eventually settle down at distant sites like the liver tissue. Our in vitro and in vivo data agreed in supporting the idea of increased metastasis formation by accelerated migration in Smad4 deficiency.

In the migration assay, we kept cells after reaching confluency under constant serum starvation to prevent proliferation interfering with intrinsic migrative power. Still, we aimed to assess the Smad4-dependent proliferative capacity in our system. In all 
CRISPR/Cas9 cells, there was no difference in proliferation of clones, thereby excluding cellular growth as an interfering factor in migration assays. This robust proliferation rate regardless of Smad4 status in vitro was counterintuitive regarding the extreme tumor growth in $\mathrm{SKC} \mathrm{fl} / \mathrm{fl} \mathrm{p} 48$ mice in in vivo. Investigating the PDAC composition by Masson's trichrome and IF stainings demonstrated that the epithelial tumor cells only represented a minor fraction of total pancreatic tissue. Importantly, the stromal compartment was significantly increased in SKC fl/fl p48 mice compared to KC p48 or SKC $\mathrm{fl} /+$ p48 mice. Excessive fibrosis caused by Smad4 depletion was already demonstrated by other laboratories (Kojima et al., 2007; Garcia-Carracedo et al., 2015). This is in agreement with Liu et al. (2017) demonstrating cancer-associated fibroblasts hijacking cancer cells for dissemination from the primary tumor site. Based on Shintani et al. (2006), we suggested that the enhanced collagen deposition in SKC fl/fl p48 mice accounts for the increased hepatic metastatic spread in low-grade, differentiated PDAC. Gene signatures associated with Smad4 deficiency as opposed to Smad4 WT identified by RNAseq with subsequent GO analysis revealed immense differences in cell morphology, proposing that important cell characteristics are decisively depending on Smad4 status. Importantly, those structural alterations were complemented by features comprising the interaction with surrounding tumor cells and stroma. GO analysis suggested that Smad4 deficiency in PDAC cells led to differential manifestation of cell-cell contacts and cell adhesion, potentially facilitating metastatic spread in vivo. In addition, our RNAseq results proposed Smad4-dependent alteration of ECM composition by affecting the secretion of chemoattractants. Smad4-deficient PDAC cells might retain their differentiated phenotype but alter their tumor microenvironment, thus attracting fibroblasts contributing to hepatic metastasis.

Another prerequisite for distant metastasis formation is anchorage-independent growth (Taddei et al., 2012). Only malignant cancer cells resist against anoikis, a special form of apoptosis caused by loss or alterations of cell adhesion (Frisch \& Francis, 1994). Yasutome et al. (2005) already described faster cell adhesion upon restoration of SMAD4 in BxPC3 cells. We observed the same phenomenon while subcultivating 
CRISPR/Cas9 clones and identified the corresponding gene signatures in our RNAseq analysis. In addition, we confirmed the crucial role of Smad4 deficiency in driving anchorage-independent growth by performing soft agar assay. The observation that sphere formation was enhanced upon loss of $\operatorname{Smad} 4$ added to the overall picture and supported the previous finding of facilitated dissemination and colony formation in distant metastasis.

In our in vitro and in vivo approaches covering different genetic backgrounds, we scrutinized the importance of Smad4 in Kras-driven PDAC. Combining all the data gathered in various models, we provided evidence for increased PDAC aggressiveness in Smad4 deficiency based on shortened lifespan and enhanced migrative potential combined with excessive fibrosis, ECM modulation, facilitated detaching and promoted dissemination contributing to improved metastasis formation. Importantly, we identified nuclear NFATc1/Smad3/cJun transcription factor assembly exclusively upon loss of Smad4. Our RNAseq studies revealed potential involvement of NFATc1/Smad3/cJun transcription complexes in driving the expression of unfavorable prognostic genes in PDAC, alteration of tumor microenvironment, and tumor cell morphology.

\subsection{Smad4 status determines chemotherapeutic response in PDAC}

Chemotherapeutic treatment of PDAC has proven to be a challenge due to minor tumor vascularization, lack of effective immune response and dense fibrotic stroma (Young et al., 2018). Since decades, Gemcitabine-based chemotherapy has been exploited for the treatment of PDAC and still prevails against the FOLFIRINOX regime because of enhanced patient tolerability despite relatively reduced efficacy (Conroy et al., 2011; Braiteh et al., 2017; Muranaka et al., 2017). Gemcitabine's toxicity is mediated by metabolizing into a nucleoside analog, thus leading to discontinuation of DNA strand replication. 
In our experimental setup, we tested Gemcitabine response in the context of serumsupplemented cultivation medium to ensure full proliferative capacity of Smad4 WT and k.o. cell clones. Indeed, we demonstrated more than twofold increase of Gemcitabine $\mathrm{IC}_{50}$ in NKC-II, KPC Bl6 and Panc-1 cells upon Smad4 deficiency, respectively. Importantly, this implicated enhanced Gemcitabine tolerance of Smad4 k.o. cells despite unaltered proliferative capacity in three different Kras-driven PDAC cell lines with different genetic background.

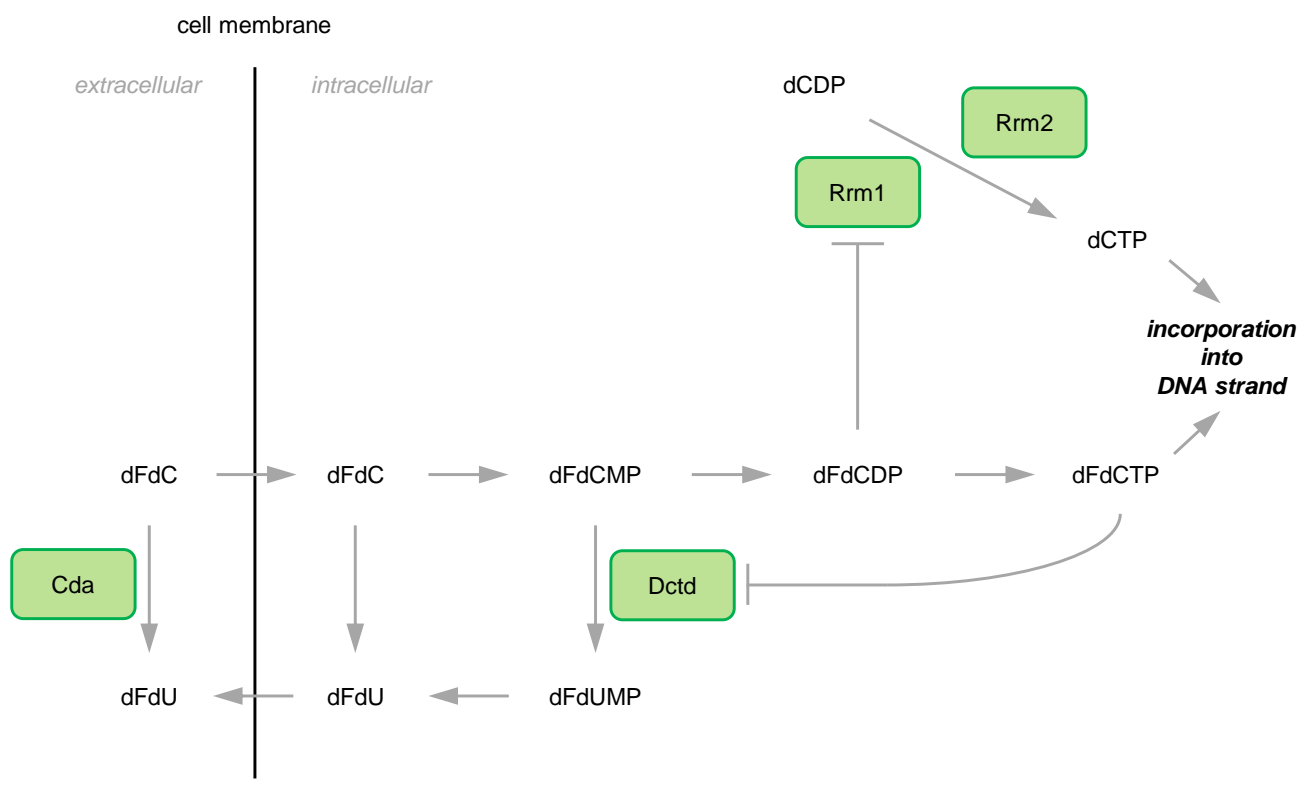

Figure 30 Smad4 deficiency interferes with Gemcitabine toxicity. Gemcitabine metabolism relies on several metabolizing steps from the prodrug $(\mathrm{dFdC})$ to the nucleoside analog dFdCTP. Smad4-deficient PDAC cells demonstrate altered regulation by Trametinib and NFATc1 silencing of the indicated enzymes in green, thus intervening with Gemcitabine metabolism on three different spots. Modified from Tanaka et al. (2010).

Additionally, we aimed to figure out if Smad4-dependent chemotherapy tolerance is a mechanism universally applicable for nucleoside analogs or specific for Gemcitabine. Therefore, we employed AraC, also known as cytarabine, for chemotherapeutic tolerance studies. We hereby confirmed the same tendency of increased AraC response in Smad4 WT Panc-1 and NKC-II cells compared to their corresponding Smad4-depleted 
counterparts, respectively. According to Li et al. (2008), the prodrugs share similar chemical structures, metabolic pathways and mechanisms of action, suggesting Smad4dependent regulation of a common regulator. Our RNAseq analysis provided insight in the regulation of genes associated with Gemcitabine and AraC metabolism by identifying four genes that are are differentially regulated by Trametinib and NFATc1 silencing upon loss of Smad4 [Figure 30]. Thus, Cda, Dctd, Rrm1, and Rrm2 are not only potentially regulated by NFATc1/Smad3/cJun transcription complexes, but are proposed mediators of increased Gemcitabine tolerance in Smad4 deficiency.

Cda plays a pivotal role in detoxifying Gemcitabine, as it converts $90 \%$ of the Gemcitabine or 2',2'-difluoro 2'-deoxycytidine (dFdC) to 2'2'-difluoro 2'-deoxyuridine (dFdU) which can be released from the cell (Neff \& Blau, 1996; Eliopoulos et al., 1998; Ueno et al., 2007). Samulitis et al. (2015) described Cda upregulation upon induced Gemcitabine resistance which was accompanied by increased invasive and metastatic potential of PDAC cells. Dctd deaminates Gemcitabine monophosphate (dFdCMP) to 2'2'difluoro 2'-deoxyuridine monophosphate (dFdUMP) but is inhibited by Gemcitabine triphosphate $(\mathrm{dFdCTP})$, the final metabolite for DNA implementation (Heinemann et al., 1992; Mini et al., 2006). In contrast, Rrm1 and Rrm2 do not interfere with Gemcitabine metabolism, but convert ribonucleosides to deoxyribonucleoside triphosphates which compete with dFdCTP in DNA strand assembly (Cory \& Sato, 1983; Nakano et al., 2007). In fact, Shukla et al. (2017) revealed that deoxycytidine nucleotide levels positively correlate with Gemcitabine $\mathrm{IC}_{50}$ in a panel of 15 PDAC cell lines. Rrm1 was identified as a mediator of induced Gemcitabine resistance in a murine in vivo model of colon cancer as well as in vitro in pancreatic cancer (Nakahira et al., 2007). Accordingly, various papers described Rrm1 as a predictive marker of Gemcitabine response in non-small-cell lung carcinoma (Rosell et al., 2004; Davidson et al., 2004; Bepler et al., 2006) and breast cancer (Rha et al., 2007). However, in pancreatic cancer neither Dctd nor Rrm1 showed any prognostic value (Elander et al., 2018). Rajabpour et al. (2017) demonstrated suppressed downregulation of Rrm1 and Cda by miR-608 in pancreatic cancer, resulting in Gemcitabine resistance. Besides its role 
in promoting Gemcitabine tolerance (Chen et al., 2018), Rrm2 was implicated in promoting malignant transformation and invasion (Fan et al., 1996; Zhou et al., 1998). Clinical studies on Rrm2 mRNA levels demonstrated inverse correlation with overall survival of Gemcitabine-treated PDAC patients (Itoi et al., 2007; Fujita et al., 2010). We summarize that Gemcitabine resistance occurred in various cancer cell types and mostly relied on the misregulation of more than one gene mediating the pyrimidine nucleoside analog's toxicity. Thus, differential regulation of Cda, Dctd, Rrm1, and Rrm2 interfered in three different ways with Gemcitabine's toxicity and the interplay of these proteins could convey additional oncogenic potential.

Among the four genes implicated in mediating Gemcitabine toxicity, loss of Smad4 led to significant upregulation of Rrm1 and Rrm2, respectively. This was in agreement with findings from our RNAseq analysis, where we identified potential NFATc1/Smad3/cJun targets and unraveled their function via GO analysis. Importantly, the top hits were associated with deoxyribonucleotide and deoxyribonucleoside metabolism and synthesis, substantiating the strong potential of Smad4-deficient PDAC cells in counteracting nucleoside analog-based chemotherapeutic agents. In line with findings of Shukla et al. (2017), we observed strong induction of pyrimidine pathway. Santana-Codina et al. (2018) revealed that excess supply of deoxyribonucleosides prevented cell death in Kras-driven PDAC cells. Increased supply of nucleotides in addition to upregulation of DNA replication and cell cycle propagation would suggest an increased proliferation rate in Smad4-deficient cells. Surprisingly, we did not observe any difference in cellular growth comparing Smad4 k.o. cells to their Smad4 WT counterparts. Possible explanations include that other nutritients and elements crucial for sufficient PDAC cell growth were limited and thus restrict proliferation to the level observed in Smad4 WT cells.

It is important to note that induction of Gemcitabine resistance in KPC Bl6 cells by sustained exposure to the prodrug did not result in alterations of Smad4 expression, although induced resistance also relied on excessive Rrm1 and Rrm2 upregulation, 
respectively. This suggested that although Smad4 deficiency drives enhanced Gemcitabine tolerance in vitro, acquired Gemcitabine resistance is not mediated via modulation of Smad4 levels by genetic or epigenetic mechanisms although similarly employing enhanced supply of competing nucleotides. In conclusion, Smad4 deficiency provided an increased resistence against nucleoside analogs in general, but the exact mode of action remains elusive.

In contrast to systemic chemotherapy approaches using nucleoside analogs, Mek1/2 inhibition by Trametinib or UO126 met the criteria for targeted cancer therapy. In our experiments, serum-supplemented medium was not crucial to identify alterations in cell viability caused by the tyrosine kinase inhibitor Trametinib. Instead, Trametinib response relied on growth factors like TGF $\beta$, which was comprised in undefined doses in FBS. As we observed increased Trametinib effects in both NKC-II clones when cultivated in TGF $\beta$-enriched medium, we concluded that TGF $\beta$ is the driving force in mediating Mek1/2 inhibition in cultivation medium.

Importantly, we introduced Mek inhibitors that demonstrated the potential to disrupt nuclear NFATc1/Smad3/cJun complex formation in vitro. Both UO126 and Trametinib proved to successfully interfere with the transcription complex assembly, thus disrupting the promotion of oncogenic gene signature and establishing a therapeutic window for systemic chemotherapy approaches. Kretzschmar et al. (1999) introduced Erk as an important regulator of Smad3 activity through linker domain phosphorylation, while Yang et al. (2003) confirmed that UO126 treatment with subsequent Erk dephosphorylation leads to partial reduction of TGF $\beta$ responsiveness. Together with the results of Sekimoto et al. (2007) underlining the importance of different Smad3 phosphorylation sites in defining the transcriptional targets, we proposed Mek inhibition with downstream loss of Erk phosphorylation as a tool to (i) disrupt NFATc1/Smad3/cJun complex formation and (ii) restore tumor-suppressive TGF $\beta$ activity even in the absence of $\operatorname{Smad} 4$. 
The exact mechanism on how Mek inhibition disrupted nuclear NFATc1/Smad3/cJun complexes remains elusive. Knock down experiments with subsequent co-IP revealed complex disruption upon silencing of either cJun or Smad3. In addition, Smad3/cJun interaction studies in Nfatc1-depleted PDAC cells demonstrated promotion of nuclear Smad3/cJun complexes by Smad4 silencing in the absence of Nfatc1. However, if NFATc1 expression contributed to Smad3/cJun complex stability or further promoted Smad3/cJun assembly was not addressed in this study. The observed Smad4 dependency suggested involvement of the carboxy-terminal MH2 domain of Smad3 in cJun binding, which needs to be confirmed in further experiments.

Mek inhibitors have been shown to reduce cJun binding to DNA (Adiseshaiah et al., 2008) and interfere with its expression levels (Favata et al., 1998). This expands the effect of Mek inhibition on cell growth, survival, adhesion, and differentiation to cJunmediated proliferation and apoptosis (Rožanc et al., 2018). However, cJun can either suppress or promote tumor development, depending on the cell type, tumor stage, differentiation, and the genetic background (Eferl \& Wagner, 2003). In colon cancer, cJun drives the expression of matrix metalloproteinase 9 (Mmp9) thus accelerating invasive potential of cells (Loesch et al., 2010). This is in agreement with Cai et al. (2017) describing the importance of cJun in EMT phenotype and acquisition of motility potential in pancreatic cancer. As the inflammatory transcription factor NFATc1 modulates Mmp13 expression (Baumgart et al., 2014), we suggest synergistic cooperativity of both transcriptional modulators in an inflammatory tumor microenvironment. This is in agreement with Chen et al. (2015), demonstrating a huge overlap of AP-1 and NFATc1-regulated gene signatures in PDAC. Indeed, cJun was portrayed as a pro-inflammatory factor in the pancreas (Cobo et al., 2018), while Qiao et al. (2016) emphasized the role of cJun in triggering inflammation-induced breast cancer progression. Importantly, Ramsdale et al. (2015) established the link between cJun-driven mesenchymal gene signatures and consequential association with increased drug resistance in melanoma cells. 
Importantly, Smad4-deficient PDAC cells were more susceptible to Mek inhibition compared to their Smad4 WT expressing counterparts. The Mek/Erk signaling cascade is one of the four pathways downstream of Kras (Collisson et al., 2012) which show a high degree of variability and preferred signaling in different contexts (Nussinov et al., 2015). In PDAC, the Mek pathway is associated with neoplastic alterations in PDAC (Collisson et al., 2012). Our data add up to findings of Santana-Codina et al. (2018), elucidating the mechanism of maintaining nucleotide pools as a specific metabolic profile in Kras dependency. We hypothesize that Smad4 deficiency favors induction of Mapk-dependent signaling by oncogenic Kras, leading to Myc upregulation and promotion of the non-oxidative pentose phosphate pathway resulting in enforced nucleotide biosynthesis (Santana-Codina et al., 2018). Importantly, Santana-Codina et al. (2018) suggested Mek inhibition as an effective tool to antagonize pyrimidine biosynthesis, which we support in the context of Smad4 deficiency. We hypothesize that Smad4depleted cells as opposed to Smad4 WT promote oncogenic signaling via Mek, but the mechanism behind increased Mek dependency of Smad4-deficient PDAC in the context of oncogenic Kras activation remains elusive.

Kawaguchi et al. (2018) introduced the clinical potential of combinatorial Gemcitabine and Trametinib treatment in patient-derived orthotopic xenograft models, resulting in significantly reduced tumor volume in nude mice. However, clinical studies with this treatment regiment (NCT1 231 581/phase 2) in metastatic PDAC failed to proof significant supremacy in overall survival compared to Gemcitabine combined with placebo therapy (Infante et al., 2014). This discrepancy between in vitro mouse models and the human situation can at least partially be explained by the use of immunodeficient mice in the patient-derived xenograft studies. In our in vitro studies, we observed additive chemotherapeutic response of Gemcitabine and Trametinib exclusively in Smad4deficient PDAC cells. Initially Gemcitabine-tolerant Smad4-depleted PDAC cells become sensitized by supplementary Trametinib treatment, suggesting that patient stratification according to Smad4 status prior to combined Gemcitabine/Trametinib treatment might be beneficial for the survival of Smad4-deficient PDAC patients. This 
is supported by our RNAseq data demonstrating approximation of gene signature in Trametinib-treated TGF $\beta$-activated Smad4-deficient cells to corresponding Smad4 WT cells. This overall alteration in gene expression pattern does not suggest Gemcitabine hypersensitivity of NKC-II Smad4 k.o. cells subsequent to Trametinib administration, but rather resensitization to the level that is observed in associated Smad4 WT clones. Thus, we demonstrated that Trametinib administration influences the global gene expression pattern and in detail leads to resensitization to Gemcitabine by interfering with its antitumoral activity by influencing both its metabolism and incorporation into the replicating DNA strand.

However, maintenance of nucleotide biosynthesis is one mechanism to resist against Mek inhibition (Santana-Codina et al., 2018). Trametinib treatment provokes feedback activation of Ras, thus resulting in no persistent Mek inhibition (Dai et al., 2019). Interestingly, Dai et al. (2019) introduced zoledronic acid as a food and drug administration (FDA)-approved tool to overcome Trametinib resistance. Zoledronic acid was already exploited in patient-derived orthotopic xenograft studies using human PDAC samples and could effectively reduce metastatic burden in combination with Gemcitabine (Hiroshima et al., 2015). As we observed increased invasive behavior upon Smad4 deficiency in PDAC cells in vitro and enhanced metastatic burden upon homozygous loss of Smad4 in vivo, additional zoledronic acid could further restrict tumor cell dissemination combined with the cytostatic and cytotoxic properties of Trametinib and Gemcitabine, respectively. Importantly, Singh et al. (2011) scrutinized the potential of zoledronic acid to disrupt NFAT stabilization. Thus, combination of these three drugs could target NFATc1/Smad3/cJun complexes in Smad4 deficiency by interfering with both NFATc1 and cJun, while keeping TGF $\beta$ signaling intact to enable the reestablishment of tumor-suppressive gene signatures.

Drug sensitivity studies in Smad4 WT and k.o. clones focused on Mek inhibitors and nucleoside analogs, respectively. First of all, we demonstrated disruption of nuclear NFATc1/Smad3/cJun complexes upon Mek inhibition by UO126 and Trametinib, 
respectively. This was associated with increased Trametinib sensitivity of Smad4depleted PDAC cells in vitro. In contrast, we revealed increased tolerance against nucleoside analogs in Smad4 deficiency as opposed to Smad4 WT PDAC. RNAseq analyses scrutinized the expression of enzymes involved in Gemcitabine metabolism and incorporation into the DNA strand, resulting in the elucidation of four genes potentially involved in mediating selective Gemcitabine tolerance upon loss of Smad4. Both NFATc1 silencing and Trametinib administration led to downregulated expression levels of respective targets, thus resulting in increased susceptibility of Trametinibtreated Smad4-deficient PDAC cells against Gemcitabine. This mode of action suggests involvement of nuclear NFATc1/Smad3/cJun complexes in mediating increased Gemcitabine tolerance of Smad4-depleted PDAC.

\subsection{Concluding remarks}

Until today, PDAC remains a highly lethal disease due to extraordinary cancer cell plasticity and tumor heterogeneity. Frequent alterations in PDAC comprise oncogenic Kras activation (90\%) and loss of the tumor suppressor Smad4 in $55 \%$ of all patients. The frequency of Smad4 alteration makes it an interesting target to exploit Smad4associated PDAC vulnerabilities for novel targeted therapeutic approaches. Smad4 is an important mediator of TGF $\beta$-mediated tumor suppression that restrains tumor growth and progression. Smad4 depletion transforms tumor-suppressive TGF $\beta$ signaling to aggressive and invasive gene signatures accelerating disease progression, metastasis formation and thus resulting in dismal prognosis. In this study, we aimed to decipher how Smad3-mediated transcription is hijacked for fulminant oncogenic signaling. In detail, the mechanistic and functional consequences of Smad4 deficiency are studied with focus on potential NFATc1/Smad3 transcription complex signatures in PDAC. 


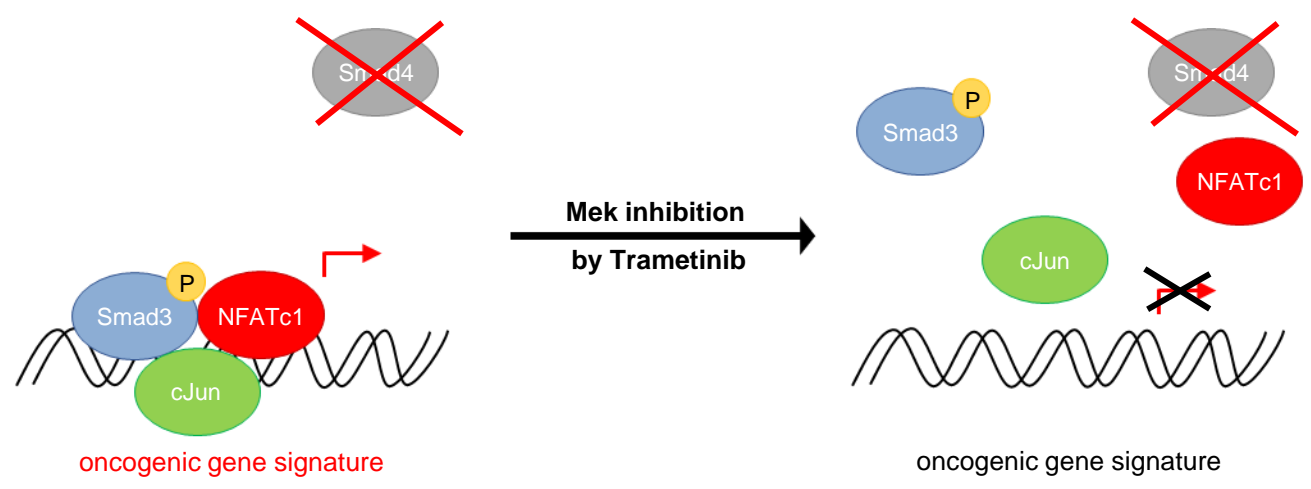

Figure 31 Trametinib targets oncogenic NFATc1/Smad3/cJun complexes in Smad4-deficient PDAC. Our current working model suggests a novel therapeutic approach for the large subgroup of Smad4-depleted PDAC patients. Loss of Smad4 favors nuclear NFATc1/Smad3/cJun transcription factor assembly promoting the expression of oncogenic gene signatures resulting in enhanced invasiveness and metastasis formation by modulation of tumor microenvironment and tumor cell morphology. Additionally, upregulation of nucleotide metabolism impairs Gemcitabine-based chemotherapy, leading together with fostered expression of proliferative genes to reduced survival of Smad4-deficient PDAC patients. Trametinib administration disrupts nuclear NFATc1/Smad3/cJun complexes, diminishes the expression of unfavorable prognostic genes in PDAC and restores Gemcitabine susceptibility for improved chemotherapeutic response.

In this work, we described the assembly of NFATc1/Smad3/cJun complexes in Smad4deficient PDAC in the context of oncogenic Kras activation. RNAseq analyses employed to disclose potential signatures of this transcription complex revealed the pivotal role of NFATc1/Smad3/cJun-driven transcription for the expression of unfavorable prognostic genes in PDAC, thus providing a potential link of NFATc1/Smad3/cJun complexes and dismal outcome of Smad4-deficient PDAC patients. Morphological alterations of Smad4-deficient PDAC cells associated with increased dissemination capacity observed in vitro and in vivo were retraced to genes differentially regulated by NFATc1/Smad3/cJun complexes. Importantly, invasion and migration potential was further driven by modulating the composition of the tumor microenvironment, facilitating metastasis formation in Smad4-deficient PDAC. Thus, we suggest that NFATc1/Smad3/cJun-regulated transcription is the main driver of the tumor- 
promoting signatures of Smad4-deficient PDAC. Additionally, we revealed increased Gemcitabine tolerance in Smad4-depleted PDAC cells which is established by enzymes potentially regulated by NFATc1/Smad3/cJun complexes. Disrupting the nuclear transcription factor complex by Trametinib treatment resulted in decrease of both unfavorable prognostic signatures and the expression of genes interfering with Gemcitabine cytotoxicity. Importantly, NFATc1/Smad3/cJun complex disassembly was significantly linked with a favorable prognosis and increased Gemcitabine sensitivity, offering a therapeutic approach in Smad4 deficiency.

The assertion of Trametinib-induced Gemcitabine resensitization of Smad4-deficient PDAC preferably needs to be tested in Smad4-depleted GEMMs as well as patientderived xenografts of SMAD4-deficient human PDAC. Both approaches aim at studying drug responses in three-dimensional tumors embedded in their respective tumor environment. This can be supplemented by short-term drug response studies of PDAC patient biopsies cultivated on media-soaked gelatin sponge (Centenera et al., 2013, 2018), enabling us to investigate the influence of therapeutic regimes directly at bulk tumor pieces containing PDAC cells, ECM, and intact tumor stroma.

We hereby propose that in future, patient stratification according to their respective SMAD4 status offers a new targeted approach in PDAC therapy. We conclude from our studies that Trametinib administration is beneficial for treatment of SMAD4-deficient PDAC patients with Gemcitabine-based chemotherapeutic regimens. 


\section{References}

Abdollah, S., Macias-Silva, M., Tsukazaki, T., Hayashi, H., Attisano, L., \& Wrana, J. L. (1997). TbetaRI Phosphorylation of Smad2 on Ser465 and Ser467 Is Required for Smad2-Smad4 Complex Formation and Signaling. Journal of Biological Chemistry, 272(44), 27678-27685.

Abe, H., Kikuchi, S., Hayakawa, K., Iida, T., Nagahashi, N., Maeda, K., Sakamoto, J., Matsumoto, N., Miura, T., Matsumura, K., Seki, N., Inaba, T., Kawasaki, H., Yamaguchi, T., Kakefuda, R., Nanayama, T., Kurachi, H., Hori, Y., Yoshida, T., Kakegawa, J., Watanabe, Y., Gilmartin, A. G., Richter, M. C., Moss, K. G., \& Laquerre, S. G. (2011). Discovery of a Highly Potent and Selective MEK Inhibitor: GSK1120212 (JTP-74057 DMSO Solvate). ACS medicinal chemistry letters, 2(4), $320-324$.

Adamska, A., Domenichini, A., \& Falasca, M. (2017). Pancreatic Ductal Adenocarcinoma: Current and Evolving Therapies. International Journal of Molecular Sciences, $18(7)$.

Adiseshaiah, P., Li, J., Vaz, M., Kalvakolanu, D. V., \& Reddy, S. P. (2008). ERK signaling regulates tumor promoter induced c-Jun recruitment at the Fra-1 promoter. Biochemical and Biophysical Research Communications, 371(2), 304-308.

Afgan, E., Baker, D., Batut, B., van den Beek, M., Bouvier, D., Cech, M., Chilton, J., Clements, D., Coraor, N., Grüning, B. A., Guerler, A., Hillman-Jackson, J., Hiltemann, S., Jalili, V., Rasche, H., Soranzo, N., Goecks, J., Taylor, J., Nekrutenko, A., \& Blankenberg, D. (2018). The Galaxy platform for accessible, reproducible and collaborative biomedical analyses: 2018 update. Nucleic Acids Research, 46(W1), W537-W544.

Anaya, J. (2016). OncoLnc: linking TCGA survival data to mRNAs, miRNAs, and lncRNAs. PeerJ. 
Anders, S. \& Huber, W. (2010). Differential expression analysis for sequence count data. Genome Biology, 11(10), R106.

Anders, S., Pyl, P. T., \& Huber, W. (2015). HTSeq-a Python framework to work with high-throughput sequencing data. Bioinformatics (Oxford, England), 31(2), 166-169.

Angel, P., Imagawa, M., Chiu, R., Stein, B., Imbra, R. J., Rahmsdorf, H. J., Jonat, C., Herrlich, P., \& Karin, M. (1987). Phorbol ester-inducible genes contain a common cis element recognized by a TPA-modulated trans-acting factor. Cell, 49(6), 729-739.

Aran, D., Sirota, M., \& Butte, A. J. (2015). Systematic pan-cancer analysis of tumour purity. Nature Communications, 6, 8971.

Ardito, C. M., Grüner, B. M., Takeuchi, K. K., Lubeseder-Martellato, C., Teichmann, N., Mazur, P. K., DelGiorno, K. E., Carpenter, E. S., Halbrook, C. J., Hall, J. C., Pal, D., Briel, T., Herner, A., Trajkovic-Arsic, M., Sipos, B., Liou, G.-Y., Storz, P., Murray, N. R., Threadgill, D. W., Sibilia, M., Washington, M. K., Wilson, C. L., Schmid, R. M., Raines, E. W., Crawford, H. C., \& Siveke, J. T. (2012). EGF Receptor Is Required for KRAS-Induced Pancreatic Tumorigenesis. Cancer Cell, (22 (3)), 304-317.

Ashburner, M., Ball, C. A., Blake, J. A., Botstein, D., Butler, H., Cherry, J. M., Davis, A. P., Dolinski, K., Dwight, S. S., Eppig, J. T., Harris, M. A., Hill, D. P., Issel-Tarver, L., Kasarskis, A., Lewis, S., Matese, J. C., Richardson, J. E., Ringwald, M., Rubin, G. M., \& Sherlock, G. (2000). Gene ontology: tool for the unification of biology. The Gene Ontology Consortium. Nature Genetics, 25(1), 25-29.

Attisano, L. \& Wrana, J. L. (2000). Smads as transcriptional co-modulators. Current Opinion in Cell Biology, 12(2), 235-243.

Aung, K. L., Fischer, S. E., Denroche, R. E., Jang, G.-H., Dodd, A., Creighton, S., Southwood, B., Liang, S.-B., Chadwick, D., Zhang, A., O’Kane, G. M., Albaba, 
H., Moura, S., Grant, R. C., Miller, J. K., Mbabaali, F., Pasternack, D., Lungu, I. M., Bartlett, J. M. S., Ghai, S., Lemire, M., Holter, S., Connor, A. A., Moffitt, R. A., Yeh, J. J., Timms, L., Krzyzanowski, P. M., Dhani, N., Hedley, D., Notta, F., Wilson, J. M., Moore, M. J., Gallinger, S., \& Knox, J. J. (2018). GenomicsDriven Precision Medicine for Advanced Pancreatic Cancer: Early Results from the COMPASS Trial. Clinical Cancer Research: An Official Journal of the American Association for Cancer Research, 24(6), 1344-1354.

Awla, D., Zetterqvist, A. V., Abdulla, A., Camello, C., Berglund, L. M., Spégel, P., Pozo, M. J., Camello, P. J., Regnér, S., Gomez, M. F., \& Thorlacius, H. (2012). NFATc3 regulates trypsinogen activation, neutrophil recruitment, and tissue damage in acute pancreatitis in mice. Gastroenterology, 143(5), 1352-1360.e7.

Bailey, P., Chang, D. K., Nones, K., Johns, A. L., Patch, A.-M., Gingras, M.-C., Miller, D. K., Christ, A. N., Bruxner, T. J. C., Quinn, M. C., Nourse, C., Murtaugh, L. C., Harliwong, I., Idrisoglu, S., Manning, S., Nourbakhsh, E., Wani, S., Fink, L., Holmes, O., Chin, V., Anderson, M. J., Kazakoff, S., Leonard, C., Newell, F., Waddell, N., Wood, S., Xu, Q., Wilson, P. J., Cloonan, N., Kassahn, K. S., Taylor, D., Quek, K., Robertson, A., Pantano, L., Mincarelli, L., Sanchez, L. N., Evers, L., Wu, J., Pinese, M., Cowley, M. J., Jones, M. D., Colvin, E. K., Nagrial, A. M., Humphrey, E. S., Chantrill, L. A., Mawson, A., Humphris, J., Chou, A., Pajic, M., Scarlett, C. J., Pinho, A. V., Giry-Laterriere, M., Rooman, I., Samra, J. S., Kench, J. G., Lovell, J. A., Merrett, N. D., Toon, C. W., Epari, K., Nguyen, N. Q., Barbour, A., Zeps, N., Moran-Jones, K., Jamieson, N. B., Graham, J. S., Duthie, F., Oien, K., Hair, J., Grützmann, R., Maitra, A., Iacobuzio-Donahue, C. A., Wolfgang, C. L., Morgan, R. A., Lawlor, R. T., Corbo, V., Bassi, C., Rusev, B., Capelli, P., Salvia, R., Tortora, G., Mukhopadhyay, D., Petersen, G. M., Australian Pancreatic Cancer Genome Initiative, Munzy, D. M., Fisher, W. E., Karim, S. A., Eshleman, J. R., Hruban, R. H., Pilarsky, C., Morton, J. P., Sansom, O. J., Scarpa, A., Musgrove, E. A., Bailey, U.-M. H., Hofmann, O., Sutherland, R. L., Wheeler, D. A., Gill, A. J., Gibbs, R. A., Pearson, J. V., Waddell, N., Biankin, A. V., \& Grimmond, S. M. 
(2016). Genomic analyses identify molecular subtypes of pancreatic cancer. Nature, $531(7592), 47-52$.

Baumgart, S., Chen, N.-M., Siveke, J. T., König, A., Zhang, J.-S., Singh, S. K., Wolf, E., Bartkuhn, M., Esposito, I., Hessmann, E., Reinecke, J., Nikorowitsch, J., Brunner, M., Singh, G., Fernandez-Zapico, M. E., Smyrk, T., Bamlet, W. R., Eilers, M., Neesse, A., Gress, T. M., Billadeau, D. D., Tuveson, D. A., Urrutia, R., \& Ellenrieder, V. (2014). Inflammation-induced NFATc1-STAT3 transcription complex promotes pancreatic cancer initiation by KrasG12d. Cancer Discovery, (4), 688-701.

Baumgart, S., Glesel, E., Singh, G., Chen, N.-M., Reutlinger, K., Zhang, J., Billadeau, D. D., Fernandez-Zapico, M. E., Gress, T. M., Singh, S. K., \& Ellenrieder, V. (2012). Restricted heterochromatin formation links NFATc2 repressor activity with growth promotion in pancreatic cancer. Gastroenterology, 142(2), 388-398.e1-7.

Ben-Neriah, Y. \& Karin, M. (2011). Inflammation meets cancer, with NFkappaB as the matchmaker. Nature Immunology, 12(8), 715-723.

Bepler, G., Kusmartseva, I., Sharma, S., Gautam, A., Cantor, A., Sharma, A., \& Simon, G. (2006). RRM1 modulated in vitro and in vivo efficacy of gemcitabine and platinum in non-small-cell lung cancer. Journal of Clinical Oncology: Official Journal of the American Society of Clinical Oncology, 24(29), 4731-4737.

Bera, A., Zhao, S., Cao, L., Chiao, P. J., \& Freeman, J. W. (2013). Oncogenic K-Ras and loss of Smad4 mediate invasion by activating an EGFR/NFkappaB Axis that induces expression of MMP9 and uPA in human pancreas progenitor cells. PloS One, 8(12), e82282.

Bhowmick, N. A., Zent, R., Ghiassi, M., McDonnell, M., \& Moses, H. L. (2001). Integrin beta 1 signaling is necessary for transforming growth factor-beta activation of p38mapk and epithelial plasticity. The Journal of Biological Chemistry, 276(50), 46707-46713. 
Biankin, A. V., Waddell, N., Kassahn, K. S., Gingras, M.-C., Muthuswamy, L. B., Johns, A. L., Miller, D. K., Wilson, P. J., Patch, A.-M., Wu, J., Chang, D. K., Cowley, M. J., Gardiner, B. B., Song, S., Harliwong, I., Idrisoglu, S., Nourse, C., Nourbakhsh, E., Manning, S., Wani, S., Gongora, M., Pajic, M., Scarlett, C. J., Gill, A. J., Pinho, A., (...), \& Grimmond, S. (2012). Pancreatic cancer genomes reveal aberrations in axon guidance pathway genes. Nature, (491), 399-405.

Bohmann, D., Bos, T. J., Admon, A., Nishimura, T., Vogt, P. K., \& Tjian, R. (1987). Human proto-oncogene c-jun encodes a DNA binding protein with structural and functional properties of transcription factor AP-1. Science (New York, N.Y.), 238(4832), 1386-1392.

Braiteh, F., Patel, M. B., Parisi, M., Ni, Q., Park, S., \& Faria, C. (2017). Comparative effectiveness and resource utilization of nab-paclitaxel plus gemcitabine vs FOLFIRINOX or gemcitabine for the first-line treatment of metastatic pancreatic adenocarcinoma in a US community setting. Cancer Management and Research, 9, 141-148.

Bryant, K. L., Mancias, J. D., Kimmelman, A. C., \& Der, C. J. (2014). KRAS: feeding pancreatic cancer proliferation. Trends in Biochemical Sciences, (39 (2)), 91-100.

Buchholz, M., Schatz, A., Wagner, M., Michl, P., Linhart, T., Adler, G., Gress, T. M., \& Ellenrieder, V. (2006). Overexpression of c-myc in pancreatic cancer caused by ectopic activation of NFATc1 and the Ca2+/calcineurin signaling pathway. The EMBO Journal, (25 (15)), 3714-3724.

Burris, H. A., Moore, M. J., Andersen, J., Green, M. R., Rothenberg, M. L., Modiano, M. R., Cripps, M. C., Portenoy, R. K., Storniolo, A. M., Tarassoff, P., Nelson, R., Dorr, F. A., Stephens, C. D., \& Von Hoff, D. D. (1997). Improvements in survival and clinical benefit with gemcitabine as first-line therapy for patients with advanced pancreas cancer: a randomized trial. Journal of Clinical Oncology: Official Journal of the American Society of Clinical Oncology, 15(6), 2403-2413. 
Cai, J., Du, S., Wang, H., Xin, B., Wang, J., Shen, W., Wei, W., Guo, Z., \& Shen, X. (2017). Tenascin-C induces migration and invasion through JNK/c-Jun signalling in pancreatic cancer. Oncotarget, 8(43), 74406-74422.

Cancer Genome Atlas Research Network (2017). Integrated Genomic Characterization of Pancreatic Ductal Adenocarcinoma. Cancer Cell, 32(2), 185-203.e13.

Cancer Genome Atlas Research Network, Weinstein, J. N., Collisson, E. A., Mills, G. B., Shaw, K. R. M., Ozenberger, B. A., Ellrott, K., Shmulevich, I., Sander, C., \& Stuart, J. M. (2013). The Cancer Genome Atlas Pan-Cancer analysis project. Nature Genetics, 45(10), 1113-1120.

Carr, R. M. \& Fernandez-Zapico, M. E. (2016). Pancreatic cancer microenvironment, to target or not to target? EMBO molecular medicine, 8(2), 80-82.

Centenera, M. M., Hickey, T. E., Jindal, S., Ryan, N. K., Ravindranathan, P., Mohammed, H., Robinson, J. L., Schiewer, M. J., Ma, S., Kapur, P., Sutherland, P. D., Hoffmann, C. E., Roehrborn, C. G., Gomella, L. G., Carroll, J. S., Birrell, S. N., Knudsen, K. E., Raj, G. V., Butler, L. M., \& Tilley, W. D. (2018). A patientderived explant (PDE) model of hormone-dependent cancer. Molecular Oncology, 12(9), 1608-1622.

Centenera, M. M., Raj, G. V., Knudsen, K. E., Tilley, W. D., \& Butler, L. M. (2013). Ex vivo culture of human prostate tissue and drug development. Nature Reviews. Urology, 10(8), 483-487.

Chen, L., Glover, J. N., Hogan, P. G., Rao, A., \& Harrison, S. C. (1998). Structure of the DNA-binding domains from NFAT, Fos and Jun bound specifically to DNA. Nature, 392(392 (6671)), 42-48.

Chen, N.-M., Neesse, A., Dyck, M. L., Steuber, B., Koenig, A. O., LubesederMartellato, C., Winter, T., Forster, T., Bohnenberger, H., Kitz, J., Reuter-Jessen, K., Griesmann, H., Gaedcke, J., Grade, M., Zhang, J.-S., Tsai, W.-C., Siveke, J., 
Schildhaus, H.-U., Ströbel, P., Johnsen, S. A., Ellenrieder, V., \& Hessmann, E. (2017). Context-Dependent Epigenetic Regulation of Nuclear Factor of Activated T Cells 1 in Pancreatic Plasticity. Gastroenterology, 152(6), 1507-1520.e15.

Chen, N.-M., Singh, G., Koenig, A., Liou, G.-Y., Storz, P., Zhang, J.-S., Regul, L., Nagarajan, S., Kühnemuth, B., Johnsen, S. A., Hebrok, M., Siveke, J., Billadeau, D. D., Ellenrieder, V., \& Hessmann, E. (2015). NFATc1 Links EGFR Signaling to Induction of Sox9 Transcription and Acinar-Ductal Transdifferentiation in the Pancreas. Gastroenterology, 148(5), 1024-1034.e9.

Chen, P., Wu, J.-N., Shu, Y., Jiang, H.-G., Zhao, X.-H., Qian, H., Chen, K., Lan, T., Chen, C.-G., \& Li, J. (2018). Gemcitabine resistance mediated by ribonucleotide reductase M2 in lung squamous cell carcinoma is reversed by GW8510 through autophagy induction. Clinical Science (London, England: 1979), 132(13), 1417-1433.

Chen, Y., Lebrun, J. J., \& Vale, W. (1996). Regulation of transforming growth factor beta- and activin-induced transcription by mammalian Mad proteins. Proceedings of the National Academy of Sciences of the United States of America, 93(23), 1299212997.

Ciacci, C., Lind, S. E., \& Podolsky, D. K. (1993). Transforming growth factor beta regulation of migration in wounded rat intestinal epithelial monolayers. Gastroenterology, 105(1), 93-101.

Clipstone, N. A. \& Crabtree, G. R. (1992). Identification of calcineurin as a key signalling enzyme in T-lymphocyte activation. Nature, (357 (6380)), 695-697.

Cobo, I., Martinelli, P., Flández, M., Bakiri, L., Zhang, M., Carrillo-de Santa-Pau, E., Jia, J., Sánchez-Arévalo Lobo, V. J., Megías, D., Felipe, I., Del Pozo, N., Millán, I., Thommesen, L., Bruland, T., Olson, S. H., Smith, J., Schoonjans, K., Bamlet, W. R., Petersen, G. M., Malats, N., Amundadottir, L. T., Wagner, E. F., \& Real, F. X. (2018). Transcriptional regulation by NR5a2 links differentiation and inflammation in the pancreas. Nature, 554(7693), 533-537. 
Collisson, E. A., Sadanandam, A., Olson, P., Gibb, W. J., Truitt, M., Gu, S., Cooc, J., Weinkle, J., Kim, G. E., Jakkula, L., Feiler, H. S., Ko, A. H., Olshen, A. B., Danenberg, K. L., Tempero, M. A., Spellman, P. T., Hanahan, D., \& Gray, J. W. (2011). Subtypes of pancreatic ductal adenocarcinoma and their differing responses to therapy. Nature Medicine, 17(4), 500-503.

Collisson, E. A., Trejo, C. L., Silva, J. M., Gu, S., Korkola, J. E., Heiser, L. M., Charles, R.-P., Rabinovich, B. A., Hann, B., Dankort, D., Spellman, P. T., Phillips, W. A., Gray, J. W., \& McMahon, M. (2012). A central role for RAF-MEK-ERK signaling in the genesis of pancreatic ductal adenocarcinoma. Cancer Discovery, 2(8), 685-693.

Conroy, T., Desseigne, F., Ychou, M., Bouché, O., Guimbaud, R., Bécouarn, Y., Adenis, A., Raoul, J.-L., Gourgou-Bourgade, S., de la Fouchardière, C., Bennouna, J., Bachet, J.-B., Khemissa-Akouz, F., Péré-Vergé, D., Delbaldo, C., Assenat, E., Chauffert, B., Michel, P., Montoto-Grillot, C., Ducreux, M., Groupe Tumeurs Digestives of Unicancer, \& PRODIGE Intergroup (2011). FOLFIRINOX versus gemcitabine for metastatic pancreatic cancer. The New England Journal of Medicine, 364(19), 1817-1825.

Cory, J. G. \& Sato, A. (1983). Regulation of ribonucleotide reductase activity in mammalian cells. Molecular and Cellular Biochemistry, 53-54(1-2), 257-266.

Dai, X., Xia, H., Zhou, S., Tang, Q., \& Bi, F. (2019). Zoledronic acid enhances the efficacy of the MEK inhibitor trametinib in KRAS mutant cancers. Cancer Letters, $442,202-212$.

Davidson, J. D., Ma, L., Flagella, M., Geeganage, S., Gelbert, L. M., \& Slapak, C. A. (2004). An increase in the expression of ribonucleotide reductase large subunit 1 is associated with gemcitabine resistance in non-small cell lung cancer cell lines. Cancer Research, 64(11), 3761-3766.

Davies, C. C., Harvey, E., McMahon, R. F. T., Finegan, K. G., Connor, F., Davis, R. J., Tuveson, D. A., \& Tournier, C. (2014). Impaired JNK signaling cooperates 
with KrasG12d expression to accelerate pancreatic ductal adenocarcinoma. Cancer Research, 74(12), 3344-3356.

de Caestecker, M. P., Parks, W. T., Frank, C. J., Castagnino, P., Bottaro, D. P., Roberts, A. B., \& Lechleider, R. J. (1998). Smad2 transduces common signals from receptor serine-threonine and tyrosine kinases. Genes $\&$ Development, 12(11), 15871592.

de Caestecker, M. P., Piek, E., \& Roberts, A. B. (2000). Role of transforming growth factor-beta signaling in cancer. Journal of the National Cancer Institute, 92(17), $1388-1402$.

De La O, J.-P., Emerson, L. L., Goodman, J. L., Froebe, S. C., Illum, B. E., Curtis, A. B., \& Murtaugh, L. C. (2008). Notch and Kras reprogram pancreatic acinar cells to ductal intraepithelial neoplasia. Proceedings of the National Academy of Sciences of the United States of America, 105(48), 18907-18912.

Dennler, S., Prunier, C., Ferrand, N., Gauthier, J.-M., \& Atfi, A. (2000). c-Jun Inhibits Transforming Growth Factor beta-mediated Transcription by Repressing Smad3 Transcriptional Activity. Journal of Biological Chemistry, 275(37), 2885828865.

Derynck, R. \& Zhang, Y. E. (2003). Smad-dependent and Smad-independent pathways in TGF-beta family signalling. Nature, 425(6958), 577-584.

di Magliano, M. P. \& Logsdon, C. D. (2013). Roles for KRAS in Pancreatic Tumor Development and Progression. Gastroenterology, (144 (6)), 1220-1229.

Diaferia, G. R., Balestrieri, C., Prosperini, E., Nicoli, P., Spaggiari, P., Zerbi, A., \& Natoli, G. (2016). Dissection of transcriptional and cis-regulatory control of differentiation in human pancreatic cancer. The EMBO journal, 35(6), 595-617.

Dreymueller, D., Theodorou, K., Donners, M., \& Ludwig, A. (2017). Fine Tuning 
Cell Migration by a Disintegrin and Metalloproteinases. Mediators of Inflammation, 2017, 9621724 .

Duda, D. G., Sunamura, M., Lefter, L. P., Furukawa, T., Yokoyama, T., Yatsuoka, T., Abe, T., Inoue, H., Motoi, F., Egawa, S.-i., Matsuno, S., \& Horii, A. (2003). Restoration of SMAD4 by gene therapy reverses the invasive phenotype in pancreatic adenocarcinoma cells. Oncogene, 22(44), 6857-6864.

Duncia, J. V., Santella, J. B., Higley, C. A., Pitts, W. J., Wityak, J., Frietze, W. E., Rankin, F. W., Sun, J. H., Earl, R. A., Tabaka, A. C., Teleha, C. A., Blom, K. F., Favata, M. F., Manos, E. J., Daulerio, A. J., Stradley, D. A., Horiuchi, K., Copeland, R. A., Scherle, P. A., Trzaskos, J. M., Magolda, R. L., Trainor, G. L., Wexler, R. R., Hobbs, F. W., \& Olson, R. E. (1998). MEK inhibitors: the chemistry and biological activity of U0126, its analogs, and cyclization products. Bioorganic 86 Medicinal Chemistry Letters, 8(20), 2839-2844.

Eferl, R. \& Wagner, E. F. (2003). AP-1: a double-edged sword in tumorigenesis. Nature Reviews. Cancer, 3(11), 859-868.

Elander, N. O., Aughton, K., Ghaneh, P., Neoptolemos, J. P., Palmer, D. H., Cox, T. F., Campbell, F., Costello, E., Halloran, C. M., Mackey, J. R., Scarfe, A. G., Valle, J. W., McDonald, A. C., Carter, R., Tebbutt, N. C., Goldstein, D., Shannon, J., Dervenis, C., Glimelius, B., Deakin, M., Charnley, R. M., Anthoney, A., Lerch, M. M., Mayerle, J., Oláh, A., Büchler, M. W., Greenhalf, W., \& European Study Group for Pancreatic Cancer (2018). Intratumoural expression of deoxycytidylate deaminase or ribonuceotide reductase subunit M1 expression are not related to survival in patients with resected pancreatic cancer given adjuvant chemotherapy. British Journal of Cancer, 118(8), 1084-1088.

Eliopoulos, N., Cournoyer, D., \& Momparler, R. L. (1998). Drug resistance to 5-aza2'-deoxycytidine, 2',2'-difluorodeoxycytidine, and cytosine arabinoside conferred by 
retroviral-mediated transfer of human cytidine deaminase cDNA into murine cells. Cancer Chemotherapy and Pharmacology, 42(5), 373-378.

Ellenrieder, V., König, A., \& Seufferlein, T. (2016). Current Standard and Future Perspectives in First- and Second-Line Treatment of Metastatic Pancreatic Adenocarcinoma. Digestion, 94(1), 44-49.

Engel, M. E., McDonnell, M. A., Law, B. K., \& Moses, H. L. (1999). Interdependent SMAD and JNK signaling in transforming growth factor-beta-mediated transcription. The Journal of Biological Chemistry, 274(52), 37413-37420.

Eppert, K., Scherer, S. W., Ozcelik, H., Pirone, R., Hoodless, P., Kim, H., Tsui, L. C., Bapat, B., Gallinger, S., Andrulis, I. L., Thomsen, G. H., Wrana, J. L., \& Attisano, L. (1996). MADR2 maps to 18q21 and encodes a TGFbeta-regulated MAD-related protein that is functionally mutated in colorectal carcinoma. Cell, 86(4), 543-552.

Eser, S., Reiff, N., Messer, M., Seidler, B., Gottschalk, K., Dobler, M., Hieber, M., Arbeiter, A., Klein, S., Kong, B., Michalski, C. W., Schlitter, A. M., Esposito, I., Kind, A. J., Rad, L., Schnieke, A. E., Baccarini, M., Alessi, D. R., Rad, R., Schmid, R. M., Schneider, G., \& Saur, D. (2013). Selective requirement of PI3k/PDK1 signaling for Kras oncogene-driven pancreatic cell plasticity and cancer. Cancer Cell, 23(3), 406-420.

Eser, S., Schnieke, A., Schneider, G., \& Saur, D. (2014). Oncogenic KRAS signalling in pancreatic cancer. British Journal of Cancer, 111(5), 817-822.

Fan, H., Villegas, C., \& Wright, J. A. (1996). Ribonucleotide reductase R2 component is a novel malignancy determinant that cooperates with activated oncogenes to determine transformation and malignant potential. Proceedings of the National Academy of Sciences of the United States of America, 93(24), 14036-14040.

Favata, M. F., Horiuchi, K. Y., Manos, E. J., Daulerio, A. J., Stradley, D. A., Feeser, W. S., Van Dyk, D. E., Pitts, W. J., Earl, R. A., Hobbs, F., Copeland, R. A., 
Magolda, R. L., Scherle, P. A., \& Trzaskos, J. M. (1998). Identification of a novel inhibitor of mitogen-activated protein kinase kinase. The Journal of Biological Chemistry, 273(29), 18623-18632.

Feldmann, G., Mishra, A., Hong, S.-M., Bisht, S., Strock, C. J., Ball, D. W., Goggins, M., Maitra, A., \& Nelkin, B. D. (2010). Inhibiting the cyclin-dependent kinase CDK5 blocks pancreatic cancer formation and progression through the suppression of Ras-Ral signaling. Cancer Research, 70(11), 4460-4469.

Feng, X. H., Zhang, Y., Wu, R. Y., \& Derynck, R. (1998). The tumor suppressor Smad4/DPC4 and transcriptional adaptor CBP/p300 are coactivators for smad3 in TGF-beta-induced transcriptional activation. Genes $\& 5$ Development, 12(14), 21532163.

Frisch, S. M. \& Francis, H. (1994). Disruption of epithelial cell-matrix interactions induces apoptosis. The Journal of Cell Biology, 124(4), 619-626.

Fujita, H., Ohuchida, K., Mizumoto, K., Itaba, S., Ito, T., Nakata, K., Yu, J., Kayashima, T., Souzaki, R., Tajiri, T., Manabe, T., Ohtsuka, T., \& Tanaka, M. (2010). Gene expression levels as predictive markers of outcome in pancreatic cancer after gemcitabine-based adjuvant chemotherapy. Neoplasia (New York, N.Y.), $12(10), 807-817$.

Fullerton, P. T., Creighton, C. J., \& Matzuk, M. M. (2015). Insights Into SMAD4 Loss in Pancreatic Cancer From Inducible Restoration of TGFbeta Signaling. Molecular Endocrinology (Baltimore, Md.), 29(10), 1440-1453.

Furukawa, F., Matsuzaki, K., Mori, S., Tahashi, Y., Yoshida, K., Sugano, Y., Yamagata, H., Matsushita, M., Seki, T., Inagaki, Y., Nishizawa, M., Fujisawa, J., \& Inoue, K. (2003). p38 MAPK mediates fibrogenic signal through Smad3 phosphorylation in rat myofibroblasts. Hepatology (Baltimore, Md.), 38(4), 879-889.

Gades, N. M., Ohash, A., Mills, L. D., Rowley, M. A., Predmore, K. S., Marler, R. J., 
\& Couch, F. J. (2008). Spontaneous vulvar papillomas in a colony of mice used for pancreatic cancer research. Comparative Medicine, 58(3), 271-275.

Gao, J., Zhu, Y., Nilsson, M., \& Sundfeldt, K. (2014). TGFbeta isoforms induce EMT independent migration of ovarian cancer cells. Cancer Cell International, 14.

Garcia-Carracedo, D., Yu, C.-C., Akhavan, N., Fine, S. A., Schönleben, F., Maehara, N., Karg, D. C., Xie, C., Qiu, W., Fine, R. L., Remotti, H. E., \& Su, G. H. (2015). Smad4 loss synergizes with TGFalpha overexpression in promoting pancreatic metaplasia, PanIN development, and fibrosis. PloS One, 10(3), e0120851.

Garcia-Cozar, F. J., Okamura, H., Aramburu, J. F., Shaw, K. T., Pelletier, L., Showalter, R., Villafranca, E., \& Rao, A. (1998). Two-site interaction of nuclear factor of activated T cells with activated calcineurin. The Journal of Biological Chemistry, 273(37), 23877-23883.

Ghosh, S. \& Chan, C.-K. K. (2016). Analysis of RNA-Seq Data Using TopHat and Cufflinks. Methods in Molecular Biology (Clifton, N.J.), 1374, 339-361.

GIMP (1997). GIMP - GNU Image Manipulation Program.

Guerra, C., Schuhmacher, A. J., Canamero, M., Grippo, P. J., Verdaguer, L., PérezGallego, L., Dubus, P., Sandgren, E. P., \& Barbacid, M. (2007). Chronic Pancreatitis Is Essential for Induction of Pancreatic Ductal Adenocarcinoma by K-Ras Oncogenes in Adult Mice. Cancer Cell, (11 (3)), 291-302.

Hahn, S. \& Kern, S. E. (1995). Molecular genetics of exocrine pancreatic neoplasms. Surgical Clinics of North America, (75 (5)), 857-869.

Hahn, S. A., Hoque, A. T., Moskaluk, C. A., da Costa, L. T., Schutte, M., Rozenblum, E., Seymour, A. B., Weinstein, C. L., Yeo, C. J., Hruban, R. H., \& Kern, S. E. (1996a). Homozygous deletion map at 18q21.1 in pancreatic cancer. Cancer Research, 56(3), 490-494. 
Hahn, S. A., Schutte, M., Hoque, A. T., Moskaluk, C. A., da Costa, L. T., Rozenblum, E., Weinstein, C. L., Fischer, A., Yeo, C. J., Hruban, R. H., \& Kern, S. E. (1996b). DPC4, a candidate tumor suppressor gene at human chromosome 18q21.1. Science (New York, N.Y.), 271(5247), 350-353.

Hanafusa, H., Ninomiya-Tsuji, J., Masuyama, N., Nishita, M., Fujisawa, J., Shibuya, H., Matsumoto, K., \& Nishida, E. (1999). Involvement of the p38 mitogen-activated protein kinase pathway in transforming growth factor-beta-induced gene expression. The Journal of Biological Chemistry, 274(38), 27161-27167.

Hartsough, M. T. \& Mulder, K. M. (1995). Transforming growth factor beta activation of p44mapk in proliferating cultures of epithelial cells. The Journal of Biological Chemistry, 270(13), 7117-7124.

Hasselluhn, M. C., Schmidt, G. E., Ellenrieder, V., Johnsen, S. A., \& Hessmann, E. (in revision). Aberrant NFATc1 signaling counteracts TGFbeta-mediated growth arrest and apoptosis induction in pancreatic cancer progression. Cell Death and Disease.

Heinemann, V., Xu, Y. Z., Chubb, S., Sen, A., Hertel, L. W., Grindey, G. B., \& Plunkett, W. (1992). Cellular elimination of 2',2'-difluorodeoxycytidine 5'-triphosphate: a mechanism of self-potentiation. Cancer Research, 52(3), 533-539.

Heldin, C. H., Miyazono, K., \& ten Dijke, P. (1997). TGF-beta signalling from cell membrane to nucleus through SMAD proteins. Nature, 390(6659), 465-471.

Hessmann, E., Zhang, J.-S., Chen, N.-M., Hasselluhn, M., Liou, G.-Y., Storz, P., Ellenrieder, V., Billadeau, D. D., \& Koenig, A. (2016). NFATc4 Regulates Sox9 Gene Expression in Acinar Cell Plasticity and Pancreatic Cancer Initiation. Stem Cells International, 2016, 5272498.

Hingorani, S. R., Petricoin, E. F., Maitra, A., Rajapakse, V., King, C., Jacobetz, M. A., Ross, S., Conrads, T. P., Veenstra, T. D., Hitt, B. A., Kawaguchi, Y., Johann, D., Liotta, L. A., Crawford, H. C., Putt, M. E., Jacks, T., Wright, C. V. E., Hruban, 
R. H., Lowy, A. M., \& Tuveson, D. A. (2003). Preinvasive and invasive ductal pancreatic cancer and its early detection in the mouse. Cancer Cell, (4 (6)), 437450.

Hingorani, S. R., Wang, L., Multani, A. S., Combs, C., Deramaudt, T., Hruban, R. H., Rustgi, A. K., Chang, S., \& Tuveson, D. A. (2005). Trp53r172h and KrasG12d cooperate to promote chromosomal instability and widely metastatic pancreatic ductal adenocarcinoma in mice. Cancer Cell, (7 (5)), 469-483.

Hiroshima, Y., Maawy, A. A., Katz, M. H. G., Fleming, J. B., Bouvet, M., Endo, I., \& Hoffman, R. M. (2015). Selective efficacy of zoledronic acid on metastasis in a patient-derived orthotopic xenograph (PDOX) nude-mouse model of human pancreatic cancer. Journal of Surgical Oncology, 111(3), 311-315.

Hocevar, B. A., Brown, T. L., \& Howe, P. H. (1999). TGF-beta induces fibronectin synthesis through a c-Jun N-terminal kinase-dependent, Smad4-independent pathway. The EMBO journal, 18(5), 1345-1356.

Hosoda, W., Chianchiano, P., Griffin, J. F., Pittman, M. E., Brosens, L. A., Noë, M., Yu, J., Shindo, K., Suenaga, M., Rezaee, N., Yonescu, R., Ning, Y., AlboresSaavedra, J., Yoshizawa, N., Harada, K., Yoshizawa, A., Hanada, K., Yonehara, S., Shimizu, M., Uehara, T., Samra, J. S., Gill, A. J., Wolfgang, C. L., Goggins, M. G., Hruban, R. H., \& Wood, L. D. (2017). Genetic analyses of isolated highgrade pancreatic intraepithelial neoplasia (HG-PanIN) reveal paucity of alterations in TP53 and SMAD4. The Journal of Pathology, 242(1), 16-23.

Iacobuzio-Donahue, C. A., Fu, B., Yachida, S., Luo, M., Abe, H., Henderson, C. M., Vilardell, F., Wang, Z., Keller, J. W., Banerjee, P., Herman, J. M., Cameron, J. L., Yeo, C. J., Halushka, M. K., Eshleman, J. R., Raben, M., Klein, A. P., Hruban, R. H., Hidalgo, M., \& Laheru, D. (2009). DPC4 gene status of the primary carcinoma correlates with patterns of failure in patients with pancreatic cancer. Journal of 
Clinical Oncology: Official Journal of the American Society of Clinical Oncology, $27(11), 1806-1813$.

Ikushima, H. \& Miyazono, K. (2010). TGFbeta signalling: a complex web in cancer progression. Nature Reviews. Cancer, 10(6), 415-424.

Imamura, T., Takase, M., Nishihara, A., Oeda, E., Hanai, J., Kawabata, M., \& Miyazono, K. (1997). Smad6 inhibits signalling by the TGF-beta superfamily. Nature, $389(6651), 622-626$.

Infante, J. R., Somer, B. G., Park, J. O., Li, C.-P., Scheulen, M. E., Kasubhai, S. M., Oh, D.-Y., Liu, Y., Redhu, S., Steplewski, K., \& Le, N. (2014). A randomised, double-blind, placebo-controlled trial of trametinib, an oral MEK inhibitor, in combination with gemcitabine for patients with untreated metastatic adenocarcinoma of the pancreas. European Journal of Cancer (Oxford, England: 1990), 50(12), 20722081.

Itoi, T., Sofuni, A., Fukushima, N., Itokawa, F., Tsuchiya, T., Kurihara, T., Moriyasu, F., Tsuchida, A., \& Kasuya, K. (2007). Ribonucleotide reductase subunit M2 mRNA expression in pretreatment biopsies obtained from unresectable pancreatic carcinomas. Journal of Gastroenterology, 42(5), 389-394.

Izeradjene, K., Combs, C., Best, M., Gopinathan, A., Wagner, A., Grady, W. M., Deng, C.-X., Hruban, R. H., Adsay, N. V., Tuveson, D. A., \& Hingorani, S. R. (2007). $\operatorname{Kras}(\mathrm{G} 12 \mathrm{~d})$ and Smad4/Dpc4 haploinsufficiency cooperate to induce mucinous cystic neoplasms and invasive adenocarcinoma of the pancreas. Cancer Cell, 11(3), 229243.

Jazag, A., Ijichi, H., Kanai, F., Imamura, T., Guleng, B., Ohta, M., Imamura, J., Tanaka, Y., Tateishi, K., Ikenoue, T., Kawakami, T., Arakawa, Y., Miyagishi, M., Taira, K., Kawabe, T., \& Omata, M. (2005). Smad4 silencing in pancreatic cancer cell lines using stable RNA interference and gene expression profiles induced by transforming growth factor-beta. Oncogene, 24(4), 662-671. 
Johnson, R., Spiegelman, B., Hanahan, D., \& Wisdom, R. (1996). Cellular transformation and malignancy induced by ras require c-jun. Molecular and Cellular Biology, 16(8), 4504-4511.

Jonckheere, N., Perrais, M., Mariette, C., Batra, S. K., Aubert, J.-P., Pigny, P., \& Van Seuningen, I. (2004). A role for human MUC4 mucin gene, the ErbB2 ligand, as a target of TGF-beta in pancreatic carcinogenesis. Oncogene, 23(34), 5729-5738.

Jones, S., Zhang, X., Parsons, D. W., Lin, J. C.-H., Leary, R. J., Angenendt, P., Mankoo, P., Carter, H., Kamiyama, H., Jimeno, A., Hong, S.-M., Fu, B., Lin, M.-T., Calhoun, E. S., Kamiyama, M., Walter, K., Nikolskaya, T., Nikolsky, Y., Hartigan, J., Smith, D. R., Hidalgo, M., Leach, S. D., Klein, A. P., Jaffee, E. M., Goggins, M., Maitra, A., Iacobuzio-Donahue, C., Eshleman, J. R., Kern, S. E., Hruban, R. H., Karchin, R., Papadopoulos, N., Parmigiani, G., Vogelstein, B., Velculescu, V. E., \& Kinzler, K. W. (2008). Core Signaling Pathways in Human Pancreatic Cancers Revealed by Global Genomic Analyses. Science, (321 (5897)), 1801-1806.

Kalluri, R. \& Zeisberg, M. (2006). Fibroblasts in cancer. Nature Reviews Cancer, (6), $392-401$.

Kawaguchi, K., Igarashi, K., Miyake, K., Lwin, T. M., Miyake, M., Kiyuna, T., Hwang, H. K., Murakami, T., Delong, J. C., Singh, S. R., Clary, B., Bouvet, M., Unno, M., \& Hoffman, R. M. (2018). MEK inhibitor trametinib in combination with gemcitabine regresses a patient-derived orthotopic xenograft (PDOX) pancreatic cancer nude mouse model. Tissue \& Cell, 52, 124-128.

Kim, D., Pertea, G., Trapnell, C., Pimentel, H., Kelley, R., \& Salzberg, S. L. (2013). TopHat2: accurate alignment of transcriptomes in the presence of insertions, deletions and gene fusions. Genome Biology, 14(4), R36.

Kleeff, J., Korc, M., Apte, M., La Vecchia, C., Johnson, C. D., Biankin, A. V., Neale, R. E., Tempero, M., Tuveson, D. A., Hruban, R. H., \& Neoptolemos, J. P. (2016). Pancreatic cancer. Nature Reviews. Disease Primers, 2, 16022. 
Kojima, K., Vickers, S. M., Adsay, N. V., Jhala, N., Kim, H.-G., Schoeb, T. R., Grizzle, W. E., \& Klug, C. A. (2007). Inactivation of Smad4 Accelerates KrasG12d-Mediated Pancreatic Neoplasia. Cancer Research, (67(17)), 8121-8130.

Kretzschmar, M., Doody, J., Timokhina, I., \& Massagué, J. (1999). A mechanism of repression of TGFbeta/ Smad signaling by oncogenic Ras. Genes $\&$ Development, $13(7), 804-816$.

Lamph, W. W., Wamsley, P., Sassone-Corsi, P., \& Verma, I. M. (1988). Induction of proto-oncogene JUN/AP-1 by serum and TPA. Nature, 334(6183), 629-631.

Leung, L., Radulovich, N., Zhu, C.-Q., Wang, D., To, C., Ibrahimov, E., \& Tsao, M.-S. (2013). Loss of canonical Smad4 signaling promotes KRAS driven malignant transformation of human pancreatic duct epithelial cells and metastasis. PloS One, 8(12), e84366.

Li, L., Fridley, B., Kalari, K., Jenkins, G., Batzler, A., Safgren, S., Hildebrandt, M., Ames, M., Schaid, D., \& Wang, L. (2008). Gemcitabine and cytosine arabinoside cytotoxicity: association with lymphoblastoid cell expression. Cancer Research, 68(17), $7050-7058$.

Liberati, N. T., Datto, M. B., Frederick, J. P., Shen, X., Wong, C., Rougier-Chapman, E. M., \& Wang, X. F. (1999). Smads bind directly to the Jun family of AP-1 transcription factors. Proceedings of the National Academy of Sciences of the United States of America, 96(9), 4844-4849.

Lim, K.-H., Baines, A. T., Fiordalisi, J. J., Shipitsin, M., Feig, L. A., Cox, A. D., Der, C. J., \& Counter, C. M. (2005). Activation of RalA is critical for Ras-induced tumorigenesis of human cells. Cancer Cell, 7(6), 533-545.

Lim, K.-H., O’Hayer, K., Adam, S. J., Kendall, S. D., Campbell, P. M., Der, C. J., \& Counter, C. M. (2006). Divergent roles for RalA and RalB in malignant growth of human pancreatic carcinoma cells. Current biology: CB, 16(24), 2385-2394. 
Liszka, L. (2014). Ductal adenocarcinoma of the pancreas usually retained SMAD4 and p53 protein status as well as expression of epithelial-to-mesenchymal transition markers and cell cycle regulators at the stage of liver metastasis. Polish Journal of Pathology, (65(2)), 100-112.

Liu, C., Zhang, Y., Lim, S., Hosaka, K., Yang, Y., Pavlova, T., Alkasalias, T., Hartman, J., Jensen, L., Xing, X., Wang, X., Lu, Y., Nie, G., \& Cao, Y. (2017). A Zebrafish Model Discovers a Novel Mechanism of Stromal Fibroblast-Mediated Cancer Metastasis. Clinical Cancer Research: An Official Journal of the American Association for Cancer Research, 23(16), 4769-4779.

Lloyd, A., Yancheva, N., \& Wasylyk, B. (1991). Transformation suppressor activity of a Jun transcription factor lacking its activation domain. Nature, 352(6336), 635-638.

Loesch, M., Zhi, H.-Y., Hou, S.-W., Qi, X.-M., Li, R.-S., Basir, Z., Iftner, T., Cuenda, A., \& Chen, G. (2010). p38gamma MAPK cooperates with c-Jun in trans-activating matrix metalloproteinase 9. The Journal of Biological Chemistry, 285(20), 1514915158.

Loh, C., Carew, J. A., Kim, J., Hogan, P. G., \& Rao, A. (1996). T-cell receptor stimulation elicits an early phase of activation and a later phase of deactivation of the transcription factor NFAT1. Molecular and Cellular Biology, 16(7), 3945-3954.

Love, M. I., Huber, W., \& Anders, S. (2014). Moderated estimation of fold change and dispersion for RNA-seq data with DESeq2. Genome Biology, 15(12), 550.

Mani, S. A., Guo, W., Liao, M.-J., Eaton, E. N., Ayyanan, A., Zhou, A. Y., Brooks, M., Reinhard, F., Zhang, C. C., Shipitsin, M., Campbell, L. L., Polyak, K., Brisken, C., Yang, J., \& Weinberg, R. A. (2008). The epithelial-mesenchymal transition generates cells with properties of stem cells. Cell, 133(4), 704-715.

Massagué, J. (2008). TGFbeta in Cancer. Cell, 134(2), 215-230. 
Massagué, J., Seoane, J., \& Wotton, D. (2005). Smad transcription factors. Genes $\&$ Development, (19(23)), 2783-2810.

Matsuura, I., Denissova, N. G., Wang, G., He, D., Long, J., \& Liu, F. (2004). Cyclin-dependent kinases regulate the antiproliferative function of Smads. Nature, 430(6996), 226-231.

Maurer, C., Holmstrom, S. R., He, J., Laise, P., Su, T., Ahmed, A., Hibshoosh, H., Chabot, J. A., Oberstein, P. E., Sepulveda, A. R., Genkinger, J. M., Zhang, J., Iuga, A. C., Bansal, M., Califano, A., \& Olive, K. P. (2019). Experimental microdissection enables functional harmonisation of pancreatic cancer subtypes. Gut.

Means, A. L., Meszoely, I. M., Suzuki, K., Miyamoto, Y., Rustgi, A. K., Coffey, R. J., Wright, C. V. E., Stoffers, D. A., \& Leach, S. D. (2005). Pancreatic epithelial plasticity mediated by acinar cell transdifferentiation and generation of nestin-positive intermediates. Development (Cambridge, England), 132(16), 3767-3776.

Melzer, C., von der Ohe, J., Hass, R., \& Ungefroren, H. (2017). TGFbeta-Dependent Growth Arrest and Cell Migration in Benign and Malignant Breast Epithelial Cells Are Antagonistically Controlled by Rac1 and Rac1b. International Journal of Molecular Sciences, 18(7).

Mi, H., Muruganujan, A., \& Thomas, P. D. (2013). PANTHER in 2013: modeling the evolution of gene function, and other gene attributes, in the context of phylogenetic trees. Nucleic Acids Research, 41(Database issue), D377-386.

Mini, E., Nobili, S., Caciagli, B., Landini, I., \& Mazzei, T. (2006). Cellular pharmacology of gemcitabine. Annals of Oncology: Official Journal of the European Society for Medical Oncology, 17 Suppl 5, v7-12.

Mishra, V. K., Subramaniam, M., Kari, V., Pitel, K. S., Baumgart, S. J., Naylor, R. M., Nagarajan, S., Wegwitz, F., Ellenrieder, V., Hawse, J. R., \& Johnsen, S. A. (2017). 
Krüppel-like Transcription Factor KLF10 Suppresses TGFbeta-Induced Epithelialto-Mesenchymal Transition via a Negative Feedback Mechanism. Cancer Research, $77(9), 2387-2400$.

Moffitt, R. A., Marayati, R., Flate, E. L., Volmar, K. E., Loeza, S. G. H., Hoadley, K. A., Rashid, N. U., Williams, L. A., Eaton, S. C., Chung, A. H., Smyla, J. K., Anderson, J. M., Kim, H. J., Bentrem, D. J., Talamonti, M. S., Iacobuzio-Donahue, C. A., Hollingsworth, M. A., \& Yeh, J. J. (2015). Virtual microdissection identifies distinct tumor- and stroma-specific subtypes of pancreatic ductal adenocarcinoma. Nature Genetics, 47(10), 1168-1178.

Mokrani, M., Klibi, J., Bluteau, D., Bismuth, G., \& Mami-Chouaib, F. (2014). Smad and NFAT pathways cooperate to induce CD103 expression in human CD8 T lymphocytes. Journal of Immunology (Baltimore, Md.: 1950), 192(5), 2471-2479.

Mootha, V. K., Lindgren, C. M., Eriksson, K.-F., Subramanian, A., Sihag, S., Lehar, J., Puigserver, P., Carlsson, E., Ridderstråle, M., Laurila, E., Houstis, N., Daly, M. J., Patterson, N., Mesirov, J. P., Golub, T. R., Tamayo, P., Spiegelman, B., Lander, E. S., Hirschhorn, J. N., Altshuler, D., \& Groop, L. C. (2003). PGC-1alpharesponsive genes involved in oxidative phosphorylation are coordinately downregulated in human diabetes. Nature Genetics, 34(3), 267-273.

Mori, S., Matsuzaki, K., Yoshida, K., Furukawa, F., Tahashi, Y., Yamagata, H., Sekimoto, G., Seki, T., Matsui, H., Nishizawa, M., Fujisawa, J.-i., \& Okazaki, K. (2004). TGF-beta and HGF transmit the signals through JNK-dependent Smad2/3 phosphorylation at the linker regions. Oncogene, 23(44), 7416-7429.

Morris, J. P., Wang, S. C., \& Hebrok, M. (2010). KRAS, Hedgehog, Wnt and the twisted developmental biology of pancreatic ductal adenocarcinoma. Nature Reviews Cancer, (10), 683-695.

Muranaka, T., Kuwatani, M., Komatsu, Y., Sawada, K., Nakatsumi, H., Kawamoto, Y., Yuki, S., Kubota, Y., Kubo, K., Kawahata, S., Kawakubo, K., Kawakami, H., 
\& Sakamoto, N. (2017). Comparison of efficacy and toxicity of FOLFIRINOX and gemcitabine with nab-paclitaxel in unresectable pancreatic cancer. Journal of Gastrointestinal Oncology, 8(3), 566-571.

Mythreye, K. \& Blobe, G. C. (2009). The type III TGFbeta receptor regulates epithelial and cancer cell migration through beta-arrestin2-mediated activation of Cdc42. Proceedings of the National Academy of Sciences, 106(20), 8221-8226.

Nakahira, S., Nakamori, S., Tsujie, M., Takahashi, Y., Okami, J., Yoshioka, S., Yamasaki, M., Marubashi, S., Takemasa, I., Miyamoto, A., Takeda, Y., Nagano, H., Dono, K., Umeshita, K., Sakon, M., \& Monden, M. (2007). Involvement of ribonucleotide reductase M1 subunit overexpression in gemcitabine resistance of human pancreatic cancer. International Journal of Cancer, 120(6), 1355-1363.

Nakano, Y., Tanno, S., Koizumi, K., Nishikawa, T., Nakamura, K., Minoguchi, M., Izawa, T., Mizukami, Y., Okumura, T., \& Kohgo, Y. (2007). Gemcitabine chemoresistance and molecular markers associated with gemcitabine transport and metabolism in human pancreatic cancer cells. British Journal of Cancer, 96(3), 457-463.

Nakao, A., Afrakhte, M., Morén, A., Nakayama, T., Christian, J. L., Heuchel, R., Itoh, S., Kawabata, M., Heldin, N. E., Heldin, C. H., \& ten Dijke, P. (1997a). Identification of Smad7, a TGFbeta-inducible antagonist of TGF-beta signalling. Nature, 389(6651), 631-635.

Nakao, A., Imamura, T., Souchelnytskyi, S., Kawabata, M., Ishisaki, A., Oeda, E., Tamaki, K., Hanai, J., Heldin, C. H., Miyazono, K., \& ten Dijke, P. (1997b). TGFbeta receptor-mediated signalling through Smad2, Smad3 and Smad4. The EMBO journal, 16(17), 5353-5362.

Neal, J. W. \& Clipstone, N. A. (2001). Glycogen synthase kinase-3 inhibits the DNA binding activity of NFATc. The Journal of Biological Chemistry, 276(5), 3666-3673. 
Neesse, A., Michl, P., Frese, K. K., Feig, C., Cook, N., Jacobetz, M. A., Lolkema, M. P., Buchholz, M., Olive, K. P., Gress, T. M., \& Tuveson, D. A. (2011). Stromal biology and therapy in pancreatic cancer. Gut, 60(6), 861-868.

Neff, T. \& Blau, C. A. (1996). Forced expression of cytidine deaminase confers resistance to cytosine arabinoside and gemcitabine. Experimental Hematology, 24(11), 1340-1346.

Nussinov, R., Jang, H., Tsai, C.-J., Liao, T.-J., Li, S., Fushman, D., \& Zhang, J. (2017). Intrinsic protein disorder in oncogenic KRAS signaling. Cellular and molecular life sciences: CMLS, 74(17), 3245-3261.

Nussinov, R., Muratcioglu, S., Tsai, C.-J., Jang, H., Gursoy, A., \& Keskin, O. (2015). The Key Role of Calmodulin in KRAS-Driven Adenocarcinomas. Molecular cancer research: $M C R, 13(9), 1265-1273$.

Offield, M. F., Jetton, T. L., Labosky, P. A., Ray, M., Stein, R. W., Magnuson, M. A., Hogan, B. L., \& Wright, C. V. (1996). PDX-1 is required for pancreatic outgrowth and differentiation of the rostral duodenum. Development (Cambridge, England), 122(3), 983-995.

Oft, M., Peli, J., Rudaz, C., Schwarz, H., Beug, H., \& Reichmann, E. (1996). TGFbeta1 and Ha-Ras collaborate in modulating the phenotypic plasticity and invasiveness of epithelial tumor cells. Genes \& Development, 10(19), 2462-2477.

Ottenhof, N. A., de Wilde, R. F., Maitra, A., Hruban, R. H., \& Offerhaus, G. J. A. (2011). Molecular Characteristics of Pancreatic Ductal Adenocarcinoma. Pathology Research International, (2011), 1-16.

Pardali, K. \& Moustakas, A. (2007). Actions of TGF-beta as tumor suppressor and pro-metastatic factor in human cancer. Biochimica Et Biophysica Acta, 1775(1), 21-62. 
Peinado, H., Quintanilla, M., \& Cano, A. (2003). Transforming growth factor beta-1 induces snail transcription factor in epithelial cell lines: mechanisms for epithelial mesenchymal transitions. The Journal of Biological Chemistry, 278(23), 21113-21123.

Pylayeva-Gupta, Y., Grabocka, E., \& Bar-Sagi, D. (2011). RAS oncogenes: weaving a tumorigenic web. Nature Reviews Cancer, (11 (11)), 761-774.

Qiao, Y., He, H., Jonsson, P., Sinha, I., Zhao, C., \& Dahlman-Wright, K. (2016). AP-1 is a key regulator of proinflammatory cytokine TNFalpha-mediated triple-negative breast cancer progression. Journal of Biological Chemistry, 291(35), 18309-18309.

Quante, A. S., Ming, C., Rottmann, M., Engel, J., Boeck, S., Heinemann, V., Westphalen, C. B., \& Strauch, K. (2016). Projections of cancer incidence and cancerrelated deaths in Germany by 2020 and 2030. Cancer Medicine, 5(9), 2649-2656.

Rahib, L., Smith, B. D., Aizenberg, R., Rosenzweig, A. B., Fleshman, J. M., \& Matrisian, L. M. (2014). Projecting cancer incidence and deaths to 2030: the unexpected burden of thyroid, liver, and pancreas cancers in the United States. Cancer Research, 74(11), 2913-2921.

Rahman, M. S., Akhtar, N., Jamil, H. M., Banik, R. S., \& Asaduzzaman, S. M. (2015). TGFbeta/BMP signaling and other molecular events: regulation of osteoblastogenesis and bone formation. Bone Research, 3, 15005.

Rajabpour, A., Afgar, A., Mahmoodzadeh, H., Radfar, J.-E.-D., Rajaei, F., \& Teimoori-Toolabi, L. (2017). MiR-608 regulating the expression of ribonucleotide reductase M1 and cytidine deaminase is repressed through induced gemcitabine chemoresistance in pancreatic cancer cells. Cancer Chemotherapy and Pharmacology, $80(4), 765-775$.

Ramsdale, R., Jorissen, R. N., Li, F. Z., Al-Obaidi, S., Ward, T., Sheppard, K. E., Bukczynska, P. E., Young, R. J., Boyle, S. E., Shackleton, M., Bollag, G., Long, G. V., Tulchinsky, E., Rizos, H., Pearson, R. B., McArthur, G. A., Dhillon, A. S., \& 
Ferrao, P. T. (2015). The transcription cofactor c-JUN mediates phenotype switching and BRAF inhibitor resistance in melanoma. Science Signaling, 8(390), ra82.

Ran, F. A., Hsu, P., Lin, C.-Y., Gootenberg, J., Konermann, S., Trevino, A. E., Scott, D., Inoue, A., Matoba, S., Zhang, Y., \& Zhang, F. (2013a). Double Nicking by RNA-Guided CRISPR Cas9 for Enhanced Genome Editing Specificity. Cell, 154(6), $1380-1389$.

Ran, F. A., Hsu, P. D., Wright, J., Agarwala, V., Scott, D. A., \& Zhang, F. (2013b). Genome engineering using the CRISPR-Cas9 system. Nature Protocols, 8(11), 22812308.

Reichert, M. \& Rustgi, A. K. (2011). Pancreatic ductal cells in development, regeneration, and neoplasia. The Journal of Clinical Investigation, 121(12), 4572-4578.

Rha, S. Y., Jeung, H. C., Choi, Y. H., Yang, W. I., Yoo, J. H., Kim, B. S., Roh, J. K., \& Chung, H. C. (2007). An association between RRM1 haplotype and gemcitabineinduced neutropenia in breast cancer patients. The Oncologist, 12(6), 622-630.

Rosell, R., Danenberg, K. D., Alberola, V., Bepler, G., Sanchez, J. J., Camps, C., Provencio, M., Isla, D., Taron, M., Diz, P., Artal, A., \& Spanish Lung Cancer Group (2004). Ribonucleotide reductase messenger RNA expression and survival in gemcitabine/cisplatin-treated advanced non-small cell lung cancer patients. Clinical Cancer Research: An Official Journal of the American Association for Cancer Research, 10(4), 1318-1325.

Rožanc, J., Sakellaropoulos, T., Antoranz, A., Guttà, C., Podder, B., Vetma, V., Rufo, N., Agostinis, P., Pliaka, V., Sauter, T., Kulms, D., Rehm, M., \& Alexopoulos, L. G. (2018). Phosphoprotein patterns predict trametinib responsiveness and optimal trametinib sensitisation strategies in melanoma. Cell Death and Differentiation.

Ryseck, R. P., Hirai, S. I., Yaniv, M., \& Bravo, R. (1988). Transcriptional activation of c-jun during the G0/G1 transition in mouse fibroblasts. Nature, 334(6182), 535-537. 
Saldanha, A. J. (2004). Java Treeview-extensible visualization of microarray data. Bioinformatics, 20(17), 3246-3248.

Samulitis, B. K., Pond, K. W., Pond, E., Cress, A. E., Patel, H., Wisner, L., Patel, C., Dorr, R. T., \& Landowski, T. H. (2015). Gemcitabine resistant pancreatic cancer cell lines acquire an invasive phenotype with collateral hypersensitivity to histone deacetylase inhibitors. Cancer Biology \& Therapy, 16(1), 43-51.

Sano, Y., Harada, J., Tashiro, S., Gotoh-Mandeville, R., Maekawa, T., \& Ishii, S. (1999). ATF-2 is a common nuclear target of Smad and TAK1 pathways in transforming growth factor-beta signaling. The Journal of Biological Chemistry, 274(13), 8949-8957.

Santana-Codina, N., Roeth, A. A., Zhang, Y., Yang, A., Mashadova, O., Asara, J. M., Wang, X., Bronson, R. T., Lyssiotis, C. A., Ying, H., \& Kimmelman, A. C. (2018). Oncogenic KRAS supports pancreatic cancer through regulation of nucleotide synthesis. Nature Communications, 9(1), 4945.

Scheffzek, K., Ahmadian, M. R., Kabsch, W., Wiesmüller, L., Lautwein, A., Schmitz, F., \& Wittinghofer, A. (1997). The Ras-RasGAP complex: structural basis for GTPase activation and its loss in oncogenic Ras mutants. Science (New York, N. Y.), $277(5324), 333-338$.

Schindelin, J., Arganda-Carreras, I., Frise, E., Kaynig, V., Longair, M., Pietzsch, T., Preibisch, S., Rueden, C., Saalfeld, S., Schmid, B., Tinevez, J.-Y., White, D. J., Hartenstein, V., Eliceiri, K., Tomancak, P., \& Cardona, A. (2012). Fiji: an opensource platform for biological-image analysis. Nature Methods, 9(7), 676-682.

Schlichting, I., Almo, S. C., Rapp, G., Wilson, K., Petratos, K., Lentfer, A., Wittinghofer, A., Kabsch, W., Pai, E. F., \& Petsko, G. A. (1990). Time-resolved X-ray crystallographic study of the conformational change in Ha-Ras p21 protein on GTP hydrolysis. Nature, 345(6273), 309-315. 
Schutte, M., Hruban, R. H., Hedrick, L., Cho, K. R., Nadasdy, G. M., Weinstein, C. L., Bova, G. S., Isaacs, W. B., Cairns, P., Nawroz, H., Sidransky, D., Casero, R. A., Meltzer, P. S., Hahn, S. A., \& Kern, S. E. (1996). DPC4 gene in various tumor types. Cancer Research, 56(11), 2527-2530.

Schwarte-Waldhoff, I., Volpert, O. V., Bouck, N. P., Sipos, B., Hahn, S. A., KleinScory, S., Lüttges, J., Klöppel, G., Graeven, U., Eilert-Micus, C., Hintelmann, A., \& Schmiegel, W. (2000). Smad4/DPC4-mediated tumor suppression through suppression of angiogenesis. Proceedings of the National Academy of Sciences of the United States of America, 97(17), 9624-9629.

Sekimoto, G., Matsuzaki, K., Yoshida, K., Mori, S., Murata, M., Seki, T., Matsui, H., Fujisawa, J.-i., \& Okazaki, K. (2007). Reversible Smad-dependent signaling between tumor suppression and oncogenesis. Cancer Research, 67(11), 5090-5096.

Sengupta, S., Jana, S., Biswas, S., Mandal, P. K., \& Bhattacharyya, A. (2013). Cooperative involvement of NFAT and SnoN mediates transforming growth factor-beta (TGF-beta) induced EMT in metastatic breast cancer (MDA-MB 231) cells. Clinical E Experimental Metastasis, 30(8), 1019-1031.

Shaulian, E. \& Karin, M. (2001). AP-1 in cell proliferation and survival. Oncogene, 20(19), 2390-2400.

Sheridan, C. M., Heist, E. K., Beals, C. R., Crabtree, G. R., \& Gardner, P. (2002). Protein kinase A negatively modulates the nuclear accumulation of NF-ATc1 by priming for subsequent phosphorylation by glycogen synthase kinase-3. The Journal of Biological Chemistry, 277(50), 48664-48676.

Shibasaki, F., Price, E. R., Milan, D., \& McKeon, F. (1996). Role of kinases and the phosphatase calcineurin in the nuclear shuttling of transcription factor NF-AT4. Nature, 382(6589), 370-373.

Shin, S. H., Kim, H. J., Hwang, D. W., Lee, J. H., Song, K. B., Jun, E., Shim, I. K., Hong, S.-M., Kim, H. J., Park, K.-M., Lee, Y.-J., \& Kim, S. C. (2017). 
The DPC4/SMAD4 genetic status determines recurrence patterns and treatment outcomes in resected pancreatic ductal adenocarcinoma: A prospective cohort study. Oncotarget, 8(11), 17945-17959.

Shintani, Y., Hollingsworth, M. A., Wheelock, M. J., \& Johnson, K. R. (2006). Collagen I Promotes Metastasis in Pancreatic Cancer by Activating c-Jun NH2-Terminal Kinase 1 and Up-regulating N-Cadherin Expression. Cancer Research, 66(24), 1174511753.

Shukla, S. K., Purohit, V., Mehla, K., Gunda, V., Chaika, N. V., Vernucci, E., King, R. J., Abrego, J., Goode, G. D., Dasgupta, A., Illies, A. L., Gebregiworgis, T., Dai, B., Augustine, J. J., Murthy, D., Attri, K. S., Mashadova, O., Grandgenett, P. M., Powers, R., Ly, Q. P., Lazenby, A. J., Grem, J. L., Yu, F., Matés, J. M., Asara, J. M., Kim, J.-W., Hankins, J. H., Weekes, C., Hollingsworth, M. A., Serkova, N. J., Sasson, A. R., Fleming, J. B., Oliveto, J. M., Lyssiotis, C. A., Cantley, L. C., Berim, L., \& Singh, P. K. (2017). MUC1 and HIF-1alpha Signaling Crosstalk Induces Anabolic Glucose Metabolism to Impart Gemcitabine Resistance to Pancreatic Cancer. Cancer Cell, 32(3), 392.

Siegel, R. L., Miller, K. D., \& Jemal, A. (2018). Cancer statistics, 2018. CA: a cancer journal for clinicians, 68(1), 7-30.

Singh, G., Singh, S. K., Koenig, A., Reutlinger, K., Nye, M. D., Adhikary, T., Eilers, M., Gress, T. M., Fernandez-Zapico, M. E., \& Ellenrieder, V. (2010). Sequential activation of NFAT and c-Myc transcription factors mediates the TGF-beta switch from a suppressor to a promoter of cancer cell proliferation. Journal of Biological Chemistry, (285(35)), 27241-27250.

Singh, S., Hasselluhn, M. C., \& Neesse, A. (2019). A tangled tale of molecular subtypes in pancreatic cancer. Gut.

Singh, S. K., Baumgart, S., Singh, G., König, A. O., Reutlinger, K., Hofbauer, L. C., Barth, P., Gress, T. M., Lomberk, G., Urrutia, R., Fernandez-Zapico, M. E., \& 
Ellenrieder, V. (2011). Disruption of a nuclear NFATc2 protein stabilization loop confers breast and pancreatic cancer growth suppression by zoledronic acid. The Journal of Biological Chemistry, 286(33), 28761-28771.

Singh, S. K., Chen, N.-M., Hessmann, E., Siveke, J. T., Lahmann, M., Singh, G., Voelker, N., Vogt, S., Esposito, I., Schmidt, A., Brendel, C., Stiewe, T., Gaedcke, J., Mernberger, M., Crawford, H. C., Bamlet, W. R., Zhang, J.-S., Li, X.-K., Smyrk, T., Billadeau, D. D., Hebrok, M., Neesse, A., Koenig, A., \& Ellenrieder, V. (2015). Antithetical NFATc1-Sox2 and p53-miR200 signaling networks govern pancreatic cancer cell plasticity. The EMBO Journal, (34), 517-530.

Smeal, T., Binetruy, B., Mercola, D. A., Birrer, M., \& Karin, M. (1991). Oncogenic and transcriptional cooperation with Ha-Ras requires phosphorylation of c-Jun on serines 63 and 73. Nature, 354(6353), 494-496.

Song, S. Y., Gannon, M., Washington, M. K., Scoggins, C. R., Meszoely, I. M., Goldenring, J. R., Marino, C. R., Sandgren, E. P., Coffey, R. J., Wright, C. V., \& Leach, S. D. (1999). Expansion of Pdx1-expressing pancreatic epithelium and islet neogenesis in transgenic mice overexpressing transforming growth factor alpha. Gastroenterology, 117(6), 1416-1426.

Subramanian, A., Tamayo, P., Mootha, V. K., Mukherjee, S., Ebert, B. L., Gillette, M. A., Paulovich, A., Pomeroy, S. L., Golub, T. R., Lander, E. S., \& Mesirov, J. P. (2005). Gene set enrichment analysis: a knowledge-based approach for interpreting genome-wide expression profiles. Proceedings of the National Academy of Sciences of the United States of America, 102(43), 15545-15550.

Subramanian, G., Schwarz, R. E., Higgins, L., McEnroe, G., Chakravarty, S., Dugar, S., \& Reiss, M. (2004). Targeting endogenous transforming growth factor beta receptor signaling in SMAD4-deficient human pancreatic carcinoma cells inhibits their invasive phenotype. Cancer Research, 64(15), 5200-5211. 
Taddei, M. L., Giannoni, E., Fiaschi, T., \& Chiarugi, P. (2012). Anoikis: an emerging hallmark in health and diseases. The Journal of Pathology, 226(2), 380-393.

Takai, E., Tsukimoto, M., Harada, H., Sawada, K., Moriyama, Y., \& Kojima, S. (2012). Autocrine regulation of TGFbeta1-induced cell migration by exocytosis of ATP and activation of P2 receptors in human lung cancer cells. J Cell Sci, 125(21), 5051-5060.

Tanaka, M., Javle, M., Dong, X., Eng, C., Abbruzzese, J. L., \& Li, D. (2010). Gemcitabine metabolic and transporter gene polymorphisms are associated with drug toxicity and efficacy in patients with locally advanced pancreatic cancer. Cancer, 116(22), 5325-5335.

Tardif, G., Pelletier, J.-P., Fahmi, H., Hum, D., Zhang, Y., Kapoor, M., \& MartelPelletier, J. (2013). NFAT3 and TGFbeta/SMAD3 regulate the expression of miR140 in osteoarthritis. Arthritis Res Ther., 15(6).

The Gene Ontology Consortium (2017). Expansion of the Gene Ontology knowledgebase and resources. Nucleic Acids Research, 45(D1), D331-D338.

Thiery, J. P. (2003). Epithelial-mesenchymal transitions in development and pathologies. Current Opinion in Cell Biology, 15(6), 740-746.

Thomas, P. D., Kejariwal, A., Campbell, M. J., Mi, H., Diemer, K., Guo, N., Ladunga, I., Ulitsky-Lazareva, B., Muruganujan, A., Rabkin, S., Vandergriff, J. A., \& Doremieux, O. (2003). PANTHER: a browsable database of gene products organized by biological function, using curated protein family and subfamily classification. Nucleic Acids Research, 31(1), 334-341.

Tone, Y., Furuuchi, K., Kojima, Y., Tykocinski, M. L., Greene, M. I., \& Tone, M. (2008). Smad3 and NFAT cooperate to induce Foxp3 expression through its enhancer. Nature Immunology, 9(2), 194-202.

Trapnell, C., Roberts, A., Goff, L., Pertea, G., Kim, D., Kelley, D. R., Pimentel, H., Salzberg, S. L., Rinn, J. L., \& Pachter, L. (2012). Differential gene and transcript 
expression analysis of RNA-seq experiments with TopHat and Cufflinks. Nature Protocols, 7(3), 562-578.

Tufegdzic Vidakovic, A., Rueda, O. M., Vervoort, S. J., Sati Batra, A., Goldgraben, M. A., Uribe-Lewis, S., Greenwood, W., Coffer, P. J., Bruna, A., \& Caldas, C. (2015). Context-Specific Effects of TGFbeta/SMAD3 in Cancer Are Modulated by the Epigenome. Cell Reports, (13 (11)), 2480-2490.

Ueno, H., Kiyosawa, K., \& Kaniwa, N. (2007). Pharmacogenomics of gemcitabine: can genetic studies lead to tailor-made therapy? British Journal of Cancer, 97(2), 145-151.

Uhlen, M., Zhang, C., Lee, S., Sjöstedt, E., Fagerberg, L., Bidkhori, G., Benfeitas, R., Arif, M., Liu, Z., Edfors, F., Sanli, K., von Feilitzen, K., Oksvold, P., Lundberg, E., Hober, S., Nilsson, P., Mattsson, J., Schwenk, J. M., Brunnström, H., Glimelius, B., Sjöblom, T., Edqvist, P.-H., Djureinovic, D., Micke, P., Lindskog, C., Mardinoglu, A., \& Ponten, F. (2017). A pathology atlas of the human cancer transcriptome. Science (New York, N.Y.), 357(6352).

UniProt Consortium, T. (2018). UniProt: the universal protein knowledgebase. Nucleic Acids Research, 46(5), 2699-2699.

Vigil, D., Martin, T. D., Williams, F., Yeh, J. J., Campbell, S. L., \& Der, C. J. (2010). Aberrant overexpression of the Rgl2 Ral small GTPase-specific guanine nucleotide exchange factor promotes pancreatic cancer growth through Ral-dependent and Ralindependent mechanisms. The Journal of Biological Chemistry, 285(45), 3472934740 .

Vincent, D. F., Gout, J., Chuvin, N., Arfi, V., Pommier, R. M., Bertolino, P., Jonckheere, N., Ripoche, D., Kaniewski, B., Martel, S., Langlois, J.-B., Goddard-Léon, S., Colombe, A., Janier, M., Van Seuningen, I., Losson, R., Valcourt, U., Treilleux, I., Dubus, P., Bardeesy, N., \& Bartholin, L. (2012). Tif1gamma suppresses murine 
pancreatic tumoral transformation by a Smad4-independent pathway. The American Journal of Pathology, 180(6), 2214-2221.

Von Hoff, D. D., Ervin, T., Arena, F. P., Chiorean, E. G., Infante, J., Moore, M., Seay, T., Tjulandin, S. A., Ma, W. W., Saleh, M. N., Harris, M., Reni, M., Dowden, S., Laheru, D., Bahary, N., Ramanathan, R. K., Tabernero, J., Hidalgo, M., Goldstein, D., Van Cutsem, E., Wei, X., Iglesias, J., \& Renschler, M. F. (2013). Increased survival in pancreatic cancer with nab-paclitaxel plus gemcitabine. The New England Journal of Medicine, 369(18), 1691-1703.

Whittle, M. C., Izeradjene, K., Rani, P. G., Feng, L., Carlson, M. A., DelGiorno, K. E., Wood, L. D., Goggins, M., Hruban, R. H., Chang, A. E., Calses, P., Thorsen, S. M., \& Hingorani, S. R. (2015). RUNX3 Controls a Metastatic Switch in Pancreatic Ductal Adenocarcinoma. Cell, 161(6), 1345-1360.

Wilentz, R. E., Iacobuzio-Donahue, C. A., Argani, P., McCarty, D. M., Parsons, J. L., Yeo, C. J., Kern, S. E., \& Hruban, R. H. (2000a). Loss of expression of Dpc4 in pancreatic intraepithelial neoplasia: Evidence that DPC4 inactivation occurs late in neoplastic progression. Cancer Research, (60), 2002-2006.

Wilentz, R. E., Su, G. H., Dai, J. L., Sparks, A. B., Argani, P., Sohn, T. A., Yeo, C. J., Kern, S. E., \& Hruban, R. H. (2000b). Immunohistochemical labeling for dpc4 mirrors genetic status in pancreatic adenocarcinomas : a new marker of DPC4 inactivation. The American Journal of Pathology, 156(1), 37-43.

Wittinghofer, A. \& Vetter, I. R. (2011). Structure-function relationships of the G domain, a canonical switch motif. Annual Review of Biochemistry, 80, 943-971.

Wong, C., Rougier-Chapman, E. M., Frederick, J. P., Datto, M. B., Liberati, N. T., Li, J. M., \& Wang, X. F. (1999). Smad3-Smad4 and AP-1 complexes synergize in transcriptional activation of the c-Jun promoter by transforming growth factor beta. Molecular and Cellular Biology, 19(3), 1821-1830. 
Xu, H., Xiao, T., Chen, C.-H., Li, W., Meyer, C. A., Wu, Q., Wu, D., Cong, L., Zhang, F., Liu, J. S., Brown, M., \& Liu, X. S. (2015). Sequence determinants of improved CRISPR sgRNA design. Genome Research, 25(8), 1147-1157.

Yachida, S. \& Iacobuzio-Donahue, C. A. (2013). Evolution and dynamics of pancreatic cancer progression. Oncogene, 32(45), 5253-5260.

Yang, X., Li, C., Herrera, P.-L., \& Deng, C.-X. (2002). Generation of Smad4/Dpc4 conditional knockout mice. Genesis, 32(2), 80-81.

Yang, Y.-C., Piek, E., Zavadil, J., Liang, D., Xie, D., Heyer, J., Pavlidis, P., Kucherlapati, R., Roberts, A. B., \& Böttinger, E. P. (2003). Hierarchical model of gene regulation by transforming growth factor beta. Proceedings of the National Academy of Sciences of the United States of America, 100(18), 10269-10274.

Yasutome, M., Gunn, J., \& Korc, M. (2005). Restoration of Smad4 in BxPC3 pancreatic cancer cells attenuates proliferation without altering angiogenesis. Clinical \& Experimental Metastasis, 22(6), 461-473.

Ying, H., Dey, P., Yao, W., Kimmelman, A. C., Draetta, G. F., Maitra, A., \& DePinho, R. A. (2016). Genetics and biology of pancreatic ductal adenocarcinoma. Genes $\mathcal{F}$ Development, 30(4), 355-385.

Young, K., Hughes, D. J., Cunningham, D., \& Starling, N. (2018). Immunotherapy and pancreatic cancer: unique challenges and potential opportunities. Therapeutic Advances in Medical Oncology, 10, 1758835918816281.

Yu, L., Hébert, M. C., \& Zhang, Y. E. (2002). TGFbeta receptor-activated p38 MAP kinase mediates Smad-independent TGFbeta responses. The EMBO journal, 21(14), $3749-3759$.

Zawel, L., Dai, J. L., Buckhaults, P., Zhou, S., Kinzler, K. W., Vogelstein, B., \& Kern, S. E. (1998). Human Smad3 and Smad4 are sequence-specific transcription activators. Molecular Cell, 1(4), 611-617. 
Zhang, Y., Feng, X., We, R., \& Derynck, R. (1996). Receptor-associated Mad homologues synergize as effectors of the TGF-beta response. Nature, 383(6596), 168-172.

Zhang, Y., Feng, X. H., \& Derynck, R. (1998). Smad3 and Smad4 cooperate with cJun/c-Fos to mediate TGFbeta-induced transcription. Nature, 394(6696), 909-913.

Zhang, Y., Musci, T., \& Derynck, R. (1997). The tumor suppressor Smad4/DPC 4 as a central mediator of Smad function. Current Biology, (7(4)), 270-276.

Zhang, Y. E. (2009). Non-Smad pathways in TGFbeta signaling. Cell Research, 19(1), 128-139.

Zhou, B. S., Tsai, P., Ker, R., Tsai, J., Ho, R., Yu, J., Shih, J., \& Yen, Y. (1998). Overexpression of transfected human ribonucleotide reductase M2 subunit in human cancer cells enhances their invasive potential. Clinical $\&$ Experimental Metastasis, $16(1), 43-49$.

Zhu, L., Shi, G., Schmidt, C. M., Hruban, R. H., \& Konieczny, S. F. (2007). Acinar Cells Contribute to the Molecular Heterogeneity of Pancreatic Intraepithelial Neoplasia. American Journal of Pathology, (171 (1)), 263-273. 


\section{Appendix}

\section{Smad4 deficiency does not interfere with proliferation of PDAC cells}

To confirm that loss of Smad4 in PDAC cells is not associated with alterations in proliferative capacity, we performed cell counting experiments in murine NKC-II and KPC Bl6 as well as human Panc-1 clones [Figure 14 B, 32]. In all established PDAC Smad4 k.o. clones, we demonstrated maintained proliferation levels irrespective of Smad4 status. Thus, we conclude that loss of Smad4 does not influence cellular growth.
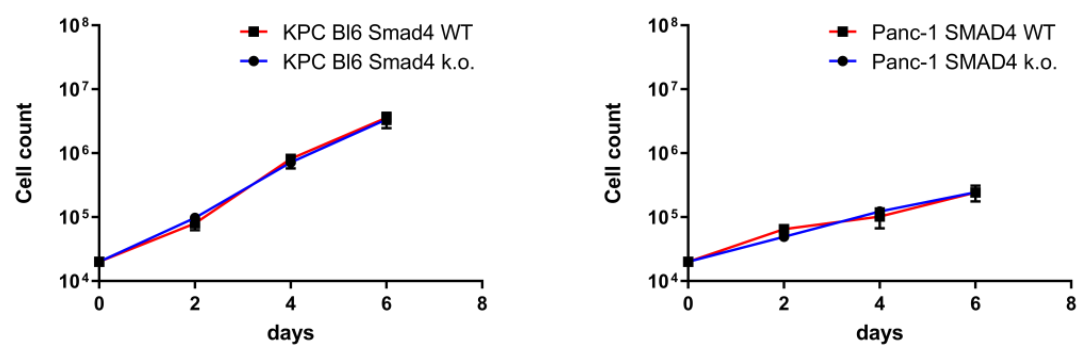

Figure 32 Proliferative capacity is not influenced by Smad4 status of PDAC cells. Murine KPC Bl6 and human Panc-1 clones were conducted to cell counting experiments to record proliferation over $6 \mathrm{~d}$ in FBS-supplemented medium. Subsequent to seeding 20000 cells in triplicates, cells were trypsinized, counted and seeded again every second day $(\mathrm{n}=3$, respectively). 


\section{Chemotherapeutic response depends on Smad4 status of PDAC cells}

Chemotherapeutic response to nucleoside analogs was tested in $72 \mathrm{~h}$ MTT approaches with cells cultivated in FBS-supplemented cultivation medium [Figure 28 A, 33]. Throughout all established Smad4 clones, we observed significantly increased tolerance against AraC and Gemcitabine upon loss of Smad4, respectively. This suggests that Smad4 deficiency differentially regulates a common enzyme mediating the cytotoxicity of both nucleoside analogs.

A

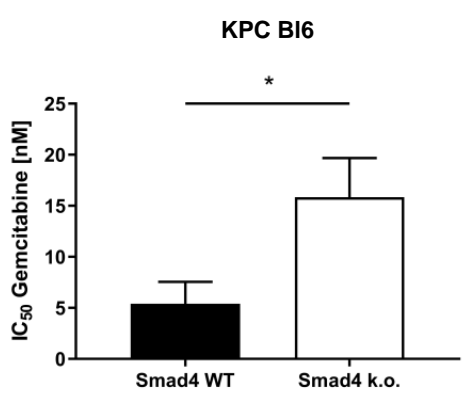

B

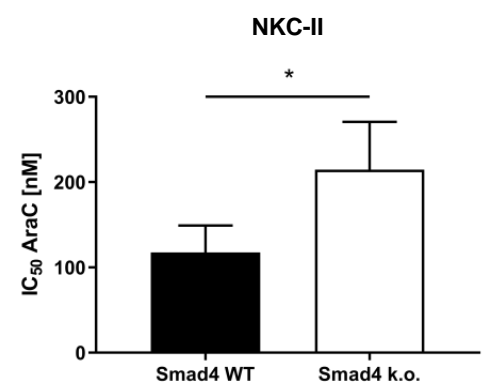

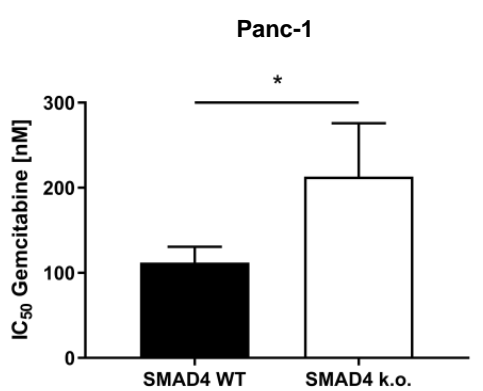

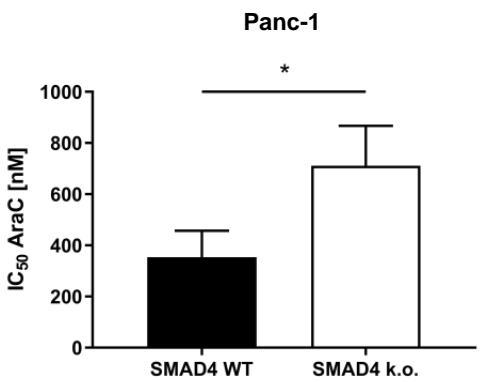

Figure 33 Smad4 deficiency enhances Gemcitabine and AraC tolerance in PDAC cells. MTT experiments were conducted in quintuplicates in a 96 well plate. Subsequent to $72 \mathrm{~h}$ incubation with the respective nucleoside analog, $\mathrm{IC}_{50}$ of independent experiments was calculated. A Murine $\operatorname{KPC} \operatorname{Bl6}(\mathrm{n}=3)$ and human Panc-1 clones $(\mathrm{n}=5)$ were tested for Gemcitabine tolerance in increasing concentrations. B AraC response was measured in murine NKC-II and human Panc-1 clones (n $=4$, respectively). 


\section{Induced Gemcitabine resistance is not associated with Smad4 silencing}

In another approach, we aimed to reveal whether Gemcitabine resistancy accomplished by consequent incubation in Gemcitabine-containing cultivation medium is achieved by Smad4 silencing. Therefore, we incubated parental KPC Bl6 cells in increasing con-

A

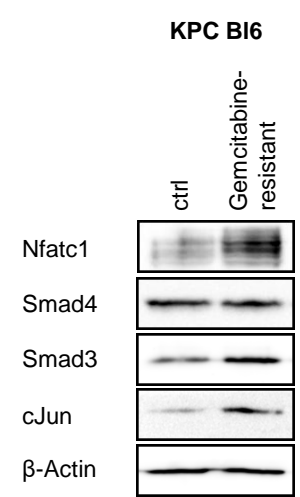

B

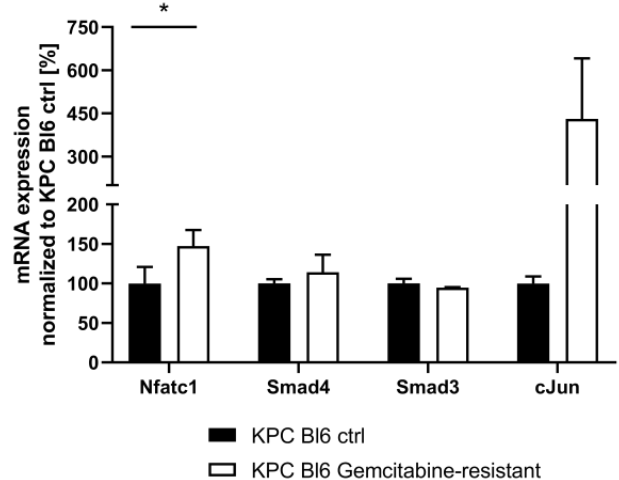

C

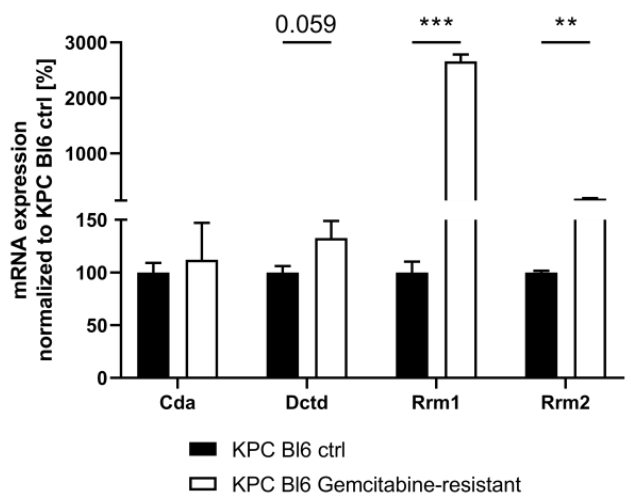

Figure 34 Induced Gemcitabine tolerance in KPC Bl6 cells is not mediated by Smad4 silencing. KPC Bl6 cells were cultivated with increasing concentrations of Gemcitabine until reaching tolerance for $200 \mathrm{nM}$ Gemcitabine. A Protein samples from KPC Bl6 control and Gemcitabine-resistant cells were conducted to western blot analysis to check for variations in Smad4 expression. B Transcription levels of Smad4, Nfatc1, Smad3 and cJun were checked subsequent to induced Gemcitabine tolerance via qRT-PCR. C Expression of previously identified genes involved in Gemcitabine metabolism were determined by qRT-PCR. 


\section{Appendix}

centrations of Gemcitabine, starting from $5 \mathrm{nM}$. Upon reaching tolerance for $200 \mathrm{nM}$, cells were further cultivated in medium containing this Gemcitabine concentration. RNA and protein of Gemcitabine-resistant and control KPC Bl6 cells were conducted to western blot and qRT-PCR studies [Figure $34 \mathrm{~A}+\mathrm{B}$ ]. Induced Gemcitabine resistance was associated with alterations in Nfatc1, Smad3 and cJun levels, respectively, but did not interfere with Smad4 protein and mRNA expression. Interestingly, Smad3 mRNA level were not induced upon Gemcitabine resistance despite strong increase of respective protein expression.

Although induced Gemcitabine restance was not mediated via Smad4 silencing, we intended to check if expression levels of previously identified enzymes were affected in the acquisition of Gemcitabine tolerance [Figure $34 \mathrm{C}$ ]. Interestingly, we detected the same strong involvement of Rrm1 and Rrm2 enzymes, and to a lesser extend, Dctd. These findings suggest that provision of an increased nucleotide supply is a common strategy to escape Gemcitabine-mediated cytotoxicity by direct competition for incorporation in the replicating DNA strand. 


\section{Acknowledgements}

Doctoral studies are associated with a lot of different impressions. Working in an international laboratory introduces you to different cultures, diverse kinds of people, and various languages. I am grateful for the opportunity to grab so many impressions and to learn about the world outside the lab while living the lab life. If a $\mathrm{PhD}$ teaches you one thing, it is resilience.

First of all, I would like to thank PD Dr. med Elisabeth Heßmann for giving me the opportunity to establish the Smad4 project in your lab. You and Prof. Volker Ellenrieder were providing the space I needed to develop not only the project but also myself during the last years. Importantly, you connected me with a lot of people, especially the Institute of Pathology. I am very thankful for the fruitful cooperation with Dr. med. Hanibal Bohnenberger and Prof. Philipp Ströbel. Furthermore, I would like to thank Prof. Heidi Hahn for constructive advice in my Thesis Advisory Committee and Prof. Michael P. Schön, Prof. Dieter Kube and Dr. Ufuk Günesdogan for joining my Examination Board.

I am extremely thankful for all the advices, tipps and support I gathered from Prof. Steve Johnsen. Although I lost you on the way to my PhD degree as part of my Thesis Advisory Committee, your outstanding way of thinking, empathetic skills \& unlimited kindness left a deep impression on me.

Particularly, I would like to thank Prof. Matthias Dobbelstein for not only agreeing to join my Thesis Advisory Committee but also for all the fruitful discussions and mental support before you even officially became part of my $\mathrm{PhD}$ adventure. Your outstanding generosity and your appreciation of hard work serve as inspiration for me and I hope to follow in your footsteps in terms of motivation. I am thankful for Antje Dickmanns and Yizhu Li's contribution to my project, your kind words \& appreciation.

Bonds were especially tight with the Johnsen lab. I am extremely glad about the cooperation with Feda Hamdan who taught me all there is to know about CRISPR/Cas9 and became a valuable friend over the years. I'll never forget the table full of food. For all the major \& minor catastrophes, Dr. Florian Wegwitz, Madhobi Sen, Xin Wang 
and Dr. Vijayalakshmi Kari provided good humor \& a helpful hand.

The Department of Gastroenterology and Gastrointestinal Oncology not only shares a corridor \& some labspace but also valuable tips and coffee breaks. The Heßmann lab would be nothing without technical support by Waltraut Kopp, Christin Kellner, and Jessica Spitalieri. Thank you for your patience \& reliability. Additionally, I am happy to have worked with my fellow PhD students Umair Latif, Zhe Zhang, Shilpa Patil, and Lennart Versemann. I would like to thank Sinan Önder and Tobias Kaiser for excellent mouse support \& a lot of laughter. I am very glad to share the lab with Benjamin C. Steuber and Lisa Knoll, who like to challenge me physically in terms of hiking \& mentally by testing my spontaneity \& repartee. I guess I can still improve all those skills. I am thankful for Jutta Blumberg's and Ulrike Wegner's support in terms of cooking \& traveling. Additionally, I would like to thank Dr. Shiv K. Singh for encouraging talks. I would like to take the chance to thank PD Dr. Dr. med. Albrecht Neeße for helping me out, giving me the chance to prove myself \& believing in me.

During my PhD, I had the chance to become part of the Dorothea Schlözer program. I am grateful I got to know Elsa Neubert, Anna-Lena Martins, and Prof. Christian Ammer who taught me a lot about motivation, appreciation, \& endurance. Especially in the last phase of $\mathrm{PhD}$, you were of inestimable value to keep me on track.

Although nobody wants to say it \& everybody neglects it, it is true: There is a life out there, outside the $\mathrm{PhD}$. A special thanks to my friends, building the support group/team Marie: Markus Allgaier, Annett Boden, Marcus Hönncher, and Lena Wichelmann. All of them contributed by being the very persons they are. Leisure is a rare commodity, making the time with you particularly valuable.

An outstanding role was held by Geske Schmidt, Andreas Mitschke and Kristina Reutlinger. Besides terrific support, hillarious laughter \& awesome advice you provided the save space I desperately needed the last months. Thank you for being such supportive people.

I also like to thank my family for the values they imparted to me, which contributed to the person I am. 